\title{
UMD-VALUED SQUARE FUNCTIONS ASSOCIATED WITH BESSEL OPERATORS IN HARDY AND BMO SPACES
}

\author{
JORGE J. BETANCOR, ALEJANDRO J. CASTRO, AND L. RODRÍGUEZ-MESA
}

\begin{abstract}
We consider Banach valued Hardy and BMO spaces in the Bessel setting. Square functions associated with Poisson semigroups for Bessel operators are defined by using fractional derivatives. If $\mathbb{B}$ is a UMD Banach space we obtain for $\mathbb{B}$-valued Hardy and BMO spaces equivalent norms involving $\gamma$-radonifying operators and square functions. We also establish characterizations of UMD Banach spaces by using Hardy and BMO-boundedness properties of $g$-functions associated to Bessel-Poisson semigroup.
\end{abstract}

\section{INTRODUCTION}

If $\left\{P_{t}\right\}_{t>0}$ denotes the classical Poisson semigroup, for every $k \in \mathbb{N}$, the $k$-th square (also called Littlewood-Paley-Stein) function $g_{k}\left(\left\{P_{t}\right\}_{t>0}\right)$ is defined by

$$
g_{k}\left(\left\{P_{t}\right\}_{t>0}\right)(f)(x)=\left(\int_{0}^{\infty}\left|t^{k} \partial_{t}^{k} P_{t}(f)(x)\right|^{2} \frac{d t}{t}\right)^{1 / 2}, \quad x \in \mathbb{R}^{n},
$$

for every $f \in L^{p}\left(\mathbb{R}^{n}\right)$. It is well-known that, for every $k \in \mathbb{N}$ and $1<p<\infty$, there exists $C>0$ such that

$$
\frac{1}{C}\|f\|_{L^{p}\left(\mathbb{R}^{n}\right)} \leq\left\|g_{k}\left(\left\{P_{t}\right\}_{t>0}\right)(f)\right\|_{L^{p}\left(\mathbb{R}^{n}\right)} \leq C\|f\|_{L^{p}\left(\mathbb{R}^{n}\right)}, \quad f \in L^{p}\left(\mathbb{R}^{n}\right),
$$

or, in other words, for every $k \in \mathbb{N}$, the norm $\|\cdot\|_{p, k}$ defined by

$$
\|f\|_{p, k}=\left\|g_{k}\left(\left\{P_{t}\right\}_{t>0}\right)(f)\right\|_{L^{p}\left(\mathbb{R}^{n}\right)}, \quad f \in L^{p}\left(\mathbb{R}^{n}\right),
$$

is equivalent to the usual norm in $L^{p}\left(\mathbb{R}^{n}\right)$. These equivalent norms $\|\cdot\|_{p, k}$ are more suitable to establish $L^{p}$-boundedness properties of certain operators (for instance, Fourier multipliers ([37, p. 58$]))$.

Square functions have been also defined for other semigroups of operators. In [37] it was developed the Littlewood-Paley theory for diffusion semigroups. If $\left\{T_{t}\right\}_{t>0}$ is a diffusion semigroup (in the sense of Stein) on the measure space $(\Omega, \Sigma, \mu)$ where $\mu$ is a $\sigma$-finite measure defined on the $\sigma$-algebra $\Sigma$ in $\Omega$, for every $k \in \mathbb{N}$, we define

$$
g_{k}\left(\left\{T_{t}\right\}_{t>0}\right)(f)(x)=\left(\int_{0}^{\infty}\left|t^{k} \partial_{t}^{k} T_{t}(f)(x)\right|^{2} \frac{d t}{t}\right)^{1 / 2}, \quad x \in \Omega,
$$

for every $f \in L^{p}(\Omega, \mu), 1<p<\infty$.

We have that, for every $k \in \mathbb{N}$ and $1<p<\infty$, there exists a constant $C>0$ such that

$$
\frac{1}{C}\left\|f-E_{0}(f)\right\|_{L^{p}(\Omega, \mu)} \leq\left\|g_{k}\left(\left\{T_{t}\right\}_{t>0}\right)(f)\right\|_{L^{p}(\Omega, \mu)} \leq C\|f\|_{L^{p}(\Omega, \mu)},
$$

Date: Tuesday $20^{\text {th }}$ November, 2018.

2010 Mathematics Subject Classification. 46E40, 42A50, 42B25, 42B35.

Key words and phrases. Square functions, Bessel operators, UMD spaces, Hardy, BMO.

The authors are partially supported by MTM2010/17974. The second author is also supported by a FPU grant from the Government of Spain. 
for every $f \in L^{p}(\Omega, \mu)([26])$. Here $E_{0}$ denotes the projector from $L^{p}(\Omega, \mu)$ to the fixed point space of $\left\{T_{t}\right\}_{t>0}$. In (37, Corollary 3, p. 121]) 14 was used to study $L^{p}$-boundedness of Laplace transform type multipliers associated to $\left\{T_{t}\right\}_{t>0}$. Spectral multipliers for a general class of operators were analyzed by Meda ([32]) by using $g$-functions .

Also, the square functions have been defined by using convolutions. Suppose that $\psi \in L^{2}\left(\mathbb{R}^{n}\right)$. We define the square function $g_{\psi}$ as follows:

$$
g_{\psi}(f)(x)=\left(\int_{0}^{\infty}\left|\psi_{t} * f(x)\right|^{2} \frac{d t}{t}\right)^{1 / 2}, \quad x \in \mathbb{R}^{n},
$$

where $\psi_{t}(x)=t^{-n} \psi(x / t), x \in \mathbb{R}^{n}$ and $t>0$. Conditions on the function $\psi$ can be given in order that the norm $\|\cdot\|_{\psi}$ defined by

$$
\|f\|_{\psi}=\left\|g_{\psi}(f)\right\|_{L^{p}\left(\mathbb{R}^{n}\right)}, \quad f \in L^{p}\left(\mathbb{R}^{n}\right),
$$

is equivalent to the usual norm in $L^{p}\left(\mathbb{R}^{n}\right)$ (see [28, Remark 2.3]). Note that the classical Poisson semigroup is a convolution semigroup.

Assume now that $\mathbb{B}$ is a Banach space and $\Omega \subset \mathbb{R}^{n}$ (for us, usually, $\Omega=\mathbb{R}$ or $\Omega=(0, \infty)$ ). For every $1 \leq p<\infty$, we denote by $L^{p}(\Omega, \mathbb{B})$ the $p$-th Bochner-Lebesgue $\mathbb{B}$-valued function space with respect to the Lebesgue measure. By $L^{1, \infty}(\Omega, \mathbb{B})$ we represent the weak $L^{1}$-Bochner-Lebesgue $\mathbb{B}$-valued function space.

Suppose that $T$ is a bounded operator from $L^{p}\left(\mathbb{R}^{n}\right)$ into itself, for some $1 \leq p<\infty$. We can define the tensor operator $T \otimes I_{\mathbb{B}}$ on $L^{p}\left(\mathbb{R}^{n}\right) \otimes \mathbb{B}$ in the natural way. Here $I_{\mathbb{B}}$ denotes the identity operator in $\mathbb{B}$. We cannot ensure that $T \otimes I_{\mathbb{B}}$ can be extended to $L^{p}\left(\mathbb{R}^{n}, \mathbb{B}\right)$ as a bounded operator in $L^{p}\left(\mathbb{R}^{n}, \mathbb{B}\right)$. However, if $T$ is a positive operator, that is, $T(f) \geq 0$ when $f \geq 0$, then $T \otimes I_{\mathbb{B}}$ can be extended from $L^{p}\left(\mathbb{R}^{n}\right) \otimes \mathbb{B}$ to $L^{p}\left(\mathbb{R}^{n}, \mathbb{B}\right)$ as a bounded operator from $L^{p}\left(\mathbb{R}^{n}, \mathbb{B}\right)$ into itself.

If $\left\{T_{t}\right\}_{t>0}$ is a diffusion semigroup, $T_{t}$ is a positive operator in $L^{p}(\Omega)$, for every $t>0$ and $1<p<\infty$. Then, for every $t>0$ the operator $T_{t} \otimes I_{\mathbb{B}}$ can be extended to $L^{p}(\Omega, \mathbb{B})$ as a bounded operator from $L^{p}(\Omega, \mathbb{B})$ into itself, for every $1<p<\infty$. We continue denoting this extension by $T_{t}$.

In order to define square functions acting on $\mathbb{B}$-valued functions the more natural way is to replace the modulus in the definitions by the norm $\|\cdot\|_{\mathbb{B}}$ in $\mathbb{B}$. We consider, for every $k \in \mathbb{N}$ and $1<p<\infty$,

$$
g_{k, \mathbb{B}}\left(\left\{P_{t}\right\}_{t>0}\right)(f)(x)=\left(\int_{0}^{\infty}\left\|t^{k} \partial_{t}^{k} P_{t}(f)(x)\right\|_{\mathbb{B}}^{2} \frac{d t}{t}\right)^{1 / 2}, \quad x \in \mathbb{R}^{n},
$$

for every $f \in L^{p}\left(\mathbb{R}^{n}, \mathbb{B}\right)$.

Kwapień [29] established that the Banach-valued version of (1) characterizes the Hilbert spaces in the following sense: $\mathbb{B}$ is isomorphic to a Hilbert space if, and only if, for some (equivalently, for every) $1<p<\infty$, there exits $C>0$ such that

$$
\frac{1}{C}\|f\|_{L^{p}\left(\mathbb{R}^{n}, \mathbb{B}\right)} \leq\left\|g_{1, \mathbb{B}}\left(\left\{P_{t}\right\}_{t>0}\right)(f)\right\|_{L^{p}\left(\mathbb{R}^{n}, \mathbb{B}\right)} \leq C\|f\|_{L^{p}\left(\mathbb{R}^{n}, \mathbb{B}\right)},
$$

for every $f \in L^{p}\left(\mathbb{R}^{n}, \mathbb{B}\right)$. For this type of vector valued $g$-functions the question is to describe the Banach spaces $\mathbb{B}$ for which one of the two inequalities in $(3)$ holds. This problem was considered in the first time by $\mathrm{Xu}$ [44] by using square functions defined by the Poisson semigroup for the torus. In [44] $\mathrm{Xu}$ introduced generalized square functions where the exponent 2 is replaced by $q \in(1, \infty)$ and he characterized the Banach spaces of $q$-martingale type and cotype as those for 
which some of the inequalities for the $q$-square function in $(3)$ holds. After Xu's results, other authors have investigated this question for vector valued $q$-square functions associated with other semigroups of operators (see [1, 13, 31] and [41, amongst others).

Hytönen [26] and Kaiser and Weis ([27] and [28]) have introduced other definitions of square functions in vector valued settings. They obtained, by using these new square functions, equivalent norms in $L^{p}\left(\mathbb{R}^{n}, \mathbb{B}\right), 1<p<\infty$, and also in the Hardy space $H^{1}\left(\mathbb{R}^{n}, \mathbb{B}\right)$ and in the bounded mean oscillation function space $B M O\left(\mathbb{R}^{n}, \mathbb{B}\right)$, provided that $\mathbb{B}$ is a UMD Banach space.

Hytönen (in [26]) defined square functions associated with subordinated diffusion semigroups in terms of stochastic integrals. Kaiser and Weis (27] and [28]) used $\gamma$-radonifying operators to get equivalent norms by employing convolution type square functions. Both approaches (stochastic integrals and $\gamma$-radonifying operators) are connected (see, for instance, [43]). In this paper, we will work with square functions involving Poisson semigroups associated with Bessel operators and we will use $\gamma$-radonifying operators.

UMD Banach spaces (as in [26], 27] and [28]) play an important role in our results. The Hilbert transform $\mathcal{H}(f)$ of $f$ is defined by

$$
\mathcal{H}(f)(x)=\lim _{\varepsilon \rightarrow 0^{+}} \frac{1}{\pi} \int_{|x-y|>\varepsilon} \frac{f(y)}{x-y} d y, \quad \text { a.e. } x \in \mathbb{R},
$$

for every $f \in L^{p}(\mathbb{R}), 1 \leq p<\infty$. It is a key result in harmonic analysis that the Hilbert transform is a bounded operator from $L^{p}(\mathbb{R})$ into itself, for every $1<p<\infty$, and from $L^{1}(\mathbb{R})$ into $L^{1, \infty}(\mathbb{R})$.

It is clear that the Hilbert transform is not a positive operator in $L^{p}(\mathbb{R}), 1 \leq p<\infty$. A Banach space $\mathbb{B}$ is said to be a UMD Banach space when the operator $\mathcal{H} \otimes I_{\mathbb{B}}$ can be extended from $L^{p}(\mathbb{R}) \otimes \mathbb{B}$ to $L^{p}(\mathbb{R}, \mathbb{B})$ as a bounded operator from $L^{p}(\mathbb{R}, \mathbb{B})$ into itself, for some $1<p<\infty$. This extension property does not depend on $1<p<\infty$ in the following sense: the extension property holds for some $1<p<\infty$ if, and only if, it is true for every $1<p<\infty$.

The main properties of UMD Banach spaces were established by Bourgain ([18]) and Burkholder ([20]). There exist a lot of characterizations for UMD Banach spaces (see, for instance, [18, [19, [20], [21], 25], [35], and [44]).

We recall now some definitions and properties concerning $\gamma$-radonifying operators. Suppose that $\left(\gamma_{k}\right)_{k \in \mathbb{N}}$ is a sequence of independent standard Gaussian random variables on a probability space $(\Omega, \Sigma, \mathbb{P}), \mathbb{B}$ is a Banach space and $H$ is a Hilbert space. If $T: H \longrightarrow \mathbb{B}$ is a linear operator we define $\|T\|_{\gamma(H, \mathbb{B})}$ as follows:

$$
\|T\|_{\gamma(H, \mathbb{B})}=\sup \left(\mathbb{E}\left\|\sum_{k} \gamma_{k} T\left(h_{k}\right)\right\|_{\mathbb{B}}^{2}\right)^{1 / 2},
$$

where the supremum is taken over all the finite ortonormal sets $\left\{h_{k}\right\}$ in H. Here $\mathbb{E}$ denotes the expectation in $(\Omega, \Sigma, \mathbb{P})$. The space $\gamma(H, \mathbb{B})$ of $\gamma$-radonifying operators from $H$ to $\mathbb{B}$ is defined as the completion, with respect to $\|\cdot\|_{\gamma(H, \mathbb{B})}$, of the space of finite rank operators in $L(H, \mathbb{B})$, the space of bounded linear operators from $H$ into $\mathbb{B}$.

If $H$ is a separable Hilbert space and the Banach space $\mathbb{B}$ does not contain copies of $c_{0}$ then

$$
\|T\|_{\gamma(H, \mathbb{B})}=\left(\mathbb{E}\left\|\sum_{k=1}^{\infty} \gamma_{k} T\left(h_{k}\right)\right\|_{\mathbb{B}}^{2}\right)^{1 / 2}, \quad T \in \gamma(H, \mathbb{B}),
$$


where $\left\{h_{k}\right\}_{k=1}^{\infty}$ is an orthonormal basis of $H$. Note that the quantity in the right hand side in the last equality does not depend of the orthonormal basis $\left\{h_{k}\right\}_{k=1}^{\infty}$ of $H$. If $\mathbb{B}$ is a UMD Banach space, $\mathbb{B}$ does not contain copies of $c_{0}$.

Suppose now that $H=L^{2}(A, \Gamma, \mu)$ where $(A, \Gamma, \mu)$ is a $\sigma$-finite measure space with countably generating $\sigma$-algebra $\Gamma$ and let $\mathbb{B}$ be a Banach space. If $f: A \longrightarrow \mathbb{B}$ is weakly continuous in $H$, that is, for every $S \in \mathbb{B}^{*}$, the dual space of $\mathbb{B}, S \circ f \in H$, then there exists $T_{f} \in L(H, \mathbb{B})$ such that

$$
\left\langle S, T_{f}(h)\right\rangle_{\mathbb{B}^{*}, \mathbb{B}}=\int_{A}\langle S, f(t)\rangle_{\mathbb{B}^{*}, \mathbb{B}} h(t) d \mu(t), \quad h \in H .
$$

It is usual to write $f \in \gamma(A, \mu, \mathbb{B})$ when $T_{f} \in \gamma(H, \mathbb{B})$ and, to simplify, to identify $f$ with $T_{f}$. If $\mathbb{B}$ does not contain copies of $c_{0}$, the space $\left\{T_{f}, f \in \gamma(A, \mu, \mathbb{B})\right\}$ is dense in $\gamma(H, \mathbb{B})$. Throughout this paper we consider $H=L^{2}((0, \infty), d t / t)$.

As a consequence of [28, Theorem 4.2] we can deduce the following result.

Theorem A. Let $\mathbb{B}$ be a UMD Banach space and $k \in \mathbb{N}$. We define

$$
G_{k, \mathbb{B}}(f)(t, x)=t^{k} \partial_{t}^{k} P_{t}(f)(x), \quad x \in \mathbb{R}^{n} \text { and } t>0,
$$

for every $f \in S\left(\mathbb{R}^{n}, \mathbb{B}\right)$, the $\mathbb{B}$-valued Schwartz function space. Then, there exists $C>0$ such that

$$
\frac{1}{C}\|f\|_{E\left(\mathbb{R}^{n}, \mathbb{B}\right)} \leq\left\|G_{k, \mathbb{B}}(f)\right\|_{E\left(\mathbb{R}^{n}, \gamma(H, \mathbb{B})\right)} \leq C\|f\|_{E\left(\mathbb{R}^{n}, \mathbb{B}\right)},
$$

where $E=L^{p}, 1<p<\infty, E=H^{1}$ or $E=B M O$.

Note that, since $\gamma(H, \mathbb{C})=H,(4)$ can be seen as a Banach valued extension of (1).

Motivated by Theorem A our objective in this paper is to obtain equivalent norms for Hardy and BMO spaces defined via Bessel operators by using square functions involving Bessel Poisson semigroups in a Banach valued setting.

The study of harmonic analysis associated with Bessel operators was started by Muckenhoupt and Stein ([33]). We consider the Bessel operator

$$
\Delta_{\lambda}=-x^{-\lambda} \frac{d}{d x} x^{2 \lambda} \frac{d}{d x} x^{-\lambda} \text { on }(0, \infty),
$$

where $\lambda>0$. The Hankel transform $h_{\lambda}$ is defined by

$$
h_{\lambda}(f)(x)=\int_{0}^{\infty} \sqrt{x y} J_{\lambda-1 / 2}(x y) f(y) d y, \quad x \in(0, \infty),
$$

for every $f \in L^{1}(0, \infty)$. By $J_{\nu}$ we denote the Bessel function of the first kind and order $\nu$. The Hankel transform can be extended from $L^{1}(0, \infty) \cap L^{2}(0, \infty)$ to $L^{2}(0, \infty)$ as an isometry in $L^{2}(0, \infty)$.

The space $S_{\lambda}(0, \infty)$ is constituted by all those smooth functions $\phi$ on $(0, \infty)$ such that, for every $m, k \in \mathbb{N}$,

$$
\eta_{m, k}^{\lambda}(\phi)=\sup _{x \in(0, \infty)}\left(1+x^{2}\right)^{m}\left|\left(\frac{1}{x} \frac{d}{d x}\right)^{k}\left(x^{-\lambda} \phi(x)\right)\right|<\infty .
$$

$S_{\lambda}(0, \infty)$ is endowed with the topology generated by the system $\left\{\eta_{m, k}^{\lambda}\right\}_{m, k \in \mathbb{N}}$ of seminorms. Thus, $S_{\lambda}(0, \infty)$ is a Fréchet space and the Hankel transform $h_{\lambda}$ is a bounded bijective operator from $S_{\lambda}(0, \infty)$ into itself and $h_{\lambda}^{-1}=h_{\lambda}$ ([45, Theorem 5.4-1]). 
The Bessel operator $\Delta_{\lambda}$ and the Hankel transform $h_{\lambda}$ are closely connected, as the following equality shows:

$$
h_{\lambda}\left(\Delta_{\lambda} f\right)(x)=x^{2} h_{\lambda}(f)(x), \quad x \in(0, \infty),
$$

for every $f \in S_{\lambda}(0, \infty)$. The Hankel transform plays for the Bessel operator the same role as the Fourier transformation with respect to the Laplacian operator.

We define the operator $\sqrt{\Delta_{\lambda}}$ as follows:

$$
\sqrt{\Delta_{\lambda}} f=h_{\lambda}\left(y h_{\lambda}(f)\right), \quad f \in D\left(\sqrt{\Delta_{\lambda}}\right),
$$

where $D\left(\sqrt{\Delta_{\lambda}}\right)=\left\{f \in L^{2}(0, \infty): y h_{\lambda}(f) \in L^{2}(0, \infty)\right\}$. The Poisson semigroup $\left\{P_{t}^{\lambda}\right\}_{t>0}$ associated with the Bessel operator $\Delta_{\lambda}$, that is, generated by the operator $-\sqrt{\Delta_{\lambda}}$, is defined by

$$
P_{t}^{\lambda}(f)(x)=\int_{0}^{\infty} P_{t}^{\lambda}(x, y) f(y) d y, \quad t, x \in(0, \infty),
$$

for every $f \in L^{p}(0, \infty), 1 \leq p \leq \infty$, where

$$
P_{t}^{\lambda}(x, y)=\frac{2 \lambda t(x y)^{\lambda}}{\pi} \int_{0}^{\pi} \frac{(\sin \theta)^{2 \lambda-1}}{\left[(x-y)^{2}+t^{2}+2 x y(1-\cos \theta)\right]^{\lambda+1}} d \theta, \quad t, x, y \in(0, \infty) .
$$

Square functions defined by the Bessel Poisson semigroup have been studied in [6], [13, [14] and [39], amongst others. In [13] it was considered generalized Littlewood-Paley functions associated with $\left\{P_{t}^{\lambda}\right\}_{t>0}$ in a Banach valued setting. If $\mathbb{B}$ is a Banach space and $1<q<\infty$ we consider the $q$-square function defined by

$$
g_{\mathbb{B}}^{q}\left(\left\{P_{t}^{\lambda}\right\}_{t>0}\right)(f)(x)=\left(\int_{0}^{\infty}\left\|t \partial_{t} P_{t}^{\lambda}(f)(x)\right\|_{\mathbb{B}}^{q} \frac{d t}{t}\right)^{1 / q}, \quad x \in(0, \infty) .
$$

By using these Littlewood-Paley functions, martingale type and cotype of the Banach space $\mathbb{B}$ can be characterized. Moreover, as in the classical case [29], according to [13, Theorems 2.4 and 2.5], if $\lambda>0$, the Banach space $\mathbb{B}$ is isomorphic to a Hilbert space if, and only if, for some (equivalently, for every) $1<p<\infty$, there exists $C>0$ such that

$$
\frac{1}{C}\|f\|_{L^{p}((0, \infty), \mathbb{B})} \leq\left\|g_{\mathbb{B}}^{2}\left(\left\{P_{t}^{\lambda}\right\}_{t>0}\right)(f)\right\|_{L^{p}(0, \infty)} \leq C\|f\|_{L^{p}((0, \infty), \mathbb{B})}, \quad f \in L^{p}((0, \infty), \mathbb{B}) .
$$

In order to extend the equivalence (5) to Banach spaces that are not Hilbert spaces we define, motivated by [28], new square functions involving Bessel Poisson semigroup and $\gamma$-radonifying operators.

In [36] Segovia and Wheeden introduced fractional derivatives as follows. Suppose that $F$ : $(0, \infty) \times \mathbb{R} \longrightarrow \mathbb{C}$ is a nice enough function, $\beta>0$ and $m \in \mathbb{N}$ is such that $m-1 \leq \beta<m$. The $\beta$-th derivative $\partial_{t}^{\beta} F$ of $F$ with respect to $t$ is defined by

$$
\partial_{t}^{\beta} F(t, x)=\frac{e^{-i \pi(m-\beta)}}{\Gamma(m-\beta)} \int_{0}^{\infty} \partial_{t}^{m} F(t+s, x) s^{m-\beta-1} d s, \quad x \in \mathbb{R} \text { and } t>0 .
$$

We consider the operator

$$
G_{\mathbb{B}}^{\lambda, \beta}(f)(t, x)=t^{\beta} \partial_{t}^{\beta} P_{t}^{\lambda}(f)(x), \quad t, x \in(0, \infty),
$$

for every $f \in L^{p}((0, \infty), \mathbb{B}), 1 \leq p \leq \infty$.

The following result was established in [6, Theorem 1.2].

Theorem B. Let $\lambda>0, \beta>0$ and $1<p<\infty$. Suppose that $\mathbb{B}$ is a UMD Banach space. Then, there exists $C>0$ such that

$$
\frac{1}{C}\|f\|_{L^{p}((0, \infty), \mathbb{B})} \leq\left\|G_{\mathbb{B}}^{\lambda, \beta}(f)\right\|_{L^{p}((0, \infty), \gamma(H, \mathbb{B}))} \leq C\|f\|_{L^{p}((0, \infty), \mathbb{B})}, \quad f \in L^{p}((0, \infty), \mathbb{B}) .
$$


Note that the Bessel Poisson semigroup $\left\{P_{t}^{\lambda}\right\}_{t>0}$ is not a diffusion semigroup because it is not conservative. Then, Theorem $\mathrm{B}$ is not included in [26, Theorem 1.6].

Our objective in this paper is to study the operator $G_{\mathbb{B}}^{\lambda, \beta}$ in Hardy and BMO spaces adapted to the Bessel setting.

Fridli [23, in his study about the local Hilbert transform considered the Hardy type space $H_{\mathrm{o}}^{1}(0, \infty)$ which consists on all those functions $f \in L^{1}(0, \infty)$ such that the odd extension function $f_{\mathrm{o}}$ of $f$ to $\mathbb{R}$ is in the classical Hardy space $H^{1}(\mathbb{R})$. In [23, Theorem 2.1] the space $H_{\mathrm{o}}^{1}(0, \infty)$ was characterized by using the local Hilbert transform.

The maximal operator $P_{*}^{\lambda}$ associated with the semigroup $\left\{P_{t}^{\lambda}\right\}_{t>0}$ is defined by

$$
P_{*}^{\lambda}(f)=\sup _{t>0}\left|P_{t}^{\lambda}(f)\right|, \quad f \in L^{p}(0, \infty), 1 \leq p \leq \infty
$$

The space $H_{\mathrm{o}}^{1}(0, \infty)$ can be described by $P_{*}^{\lambda}$ as follows (see [11, Theorem 1.10]).

Theorem C. Let $\lambda>0$ and $f \in L^{1}(0, \infty)$. Then, $f \in H_{\mathrm{o}}^{1}(0, \infty)$ if, and only if, $P_{*}^{\lambda}(f) \in$ $L^{1}(0, \infty)$.

Note that, according to Theorem C, the Hardy space defined by the Bessel Poisson semigroup $\left\{P_{t}^{\lambda}\right\}_{t>0}$ actually does not depend on $\lambda$.

If $\mathbb{B}$ is a Banach space, the $\mathbb{B}$-valued Hardy space $H_{\mathrm{o}}^{1}((0, \infty), \mathbb{B})$ is defined as in the scalar case. By considering the maximal operator $P_{*}^{\lambda}$ on $L^{p}((0, \infty), \mathbb{B}), 1 \leq p<\infty$, as follows

$$
P_{*}^{\lambda}(f)=\sup _{t>0}\left\|P_{t}^{\lambda}(f)\right\|_{\mathbb{B}}
$$

we introduce the space $H^{1}\left(\Delta_{\lambda}, \mathbb{B}\right)$ constituted by all those functions $f \in L^{1}((0, \infty), \mathbb{B})$ such that $P_{*}^{\lambda}(f) \in L^{1}(0, \infty)$. As in Theorem $\mathbb{C}$, a function $f \in L^{1}((0, \infty), \mathbb{B})$ is in $H_{\mathrm{o}}^{1}((0, \infty), \mathbb{B})$ if, and only if, $f \in H^{1}\left(\Delta_{\lambda}, \mathbb{B}\right)$ (see Proposition 2.1.

By $B M O(\mathbb{R})$ we denote as usual the space of all bounded mean oscillation functions on $\mathbb{R}$. In [9] the space $B M O_{\mathrm{o}}(0, \infty)$ was introduced. A complex measurable function $f$ on $(0, \infty)$ is said to be in $B M O_{\mathrm{o}}(0, \infty)$ when there exists $C>0$ such that:

$$
\begin{aligned}
& \text { (Bi) } \frac{1}{|I|} \int_{I}|f(x)| d x \leq C, \text { for every } I=(0, r), r>0, \\
& \text { (Bii) } \frac{1}{|I|} \int_{I}\left|f(x)-f_{I}\right| d x \leq C, \text { for each } I=(r, s), 0<r<s<\infty .
\end{aligned}
$$

Here, $|\mathrm{I}|$ denotes the length of $I$ and $f_{I}=\frac{1}{|I|} \int_{I} f(y) d y$.

Condition (Bii) says that $f \in B M O(0, \infty)$. It is not hard to see that $f \in B M O_{\mathrm{o}}(0, \infty)$ if, and only if, the odd extension $f_{\mathrm{o}}$ of $f$ to $\mathbb{R}$ is in $B M O(\mathbb{R})$. As in the classical case, the dual space of $H_{\mathrm{o}}^{1}(0, \infty)$ can be identified with the space $B M O_{\mathrm{o}}(0, \infty)$. In [8] harmonic analysis operators (maximal operators, $g$-functions, Riesz transforms, ...) in the Bessel setting were studied on $B M O_{\mathrm{o}}(0, \infty)$ and in [9], $B M O_{\mathrm{o}}(0, \infty)$ was described by using Carleson measures involving Bessel Poisson semigroups.

If $\mathbb{B}$ is a Banach space, the Banach valued function space $B M O_{\mathrm{o}}((0, \infty), \mathbb{B})$ is defined as in the scalar case, replacing the modulus by the norm $\|\cdot\|_{\mathbb{B}}$ in $\mathbb{B}$. The dual space of $H_{\mathrm{o}}^{1}((0, \infty), \mathbb{B})$ can be identified with $B M O_{\mathrm{o}}\left((0, \infty), \mathbb{B}^{*}\right)$, provided that $\mathbb{B}$ has the UMD property.

Our first result is the following. 
Theorem 1.1. Let $\lambda \geq 1$ and $\beta>0$. Suppose that $\mathbb{B}$ is a UMD Banach space. Then, there exists $C>0$ such that

$$
\frac{1}{C}\|f\|_{E((0, \infty), \mathbb{B})} \leq\left\|G_{\mathbb{B}}^{\lambda, \beta}(f)\right\|_{E((0, \infty), \gamma(H, \mathbb{B}))} \leq C\|f\|_{E((0, \infty), \mathbb{B})}, \quad f \in E((0, \infty), \mathbb{B}),
$$

where $E$ denotes $H_{\mathrm{O}}^{1}$ or $B M O_{\mathrm{o}}$.

The Bessel operator $\Delta_{\lambda}$ can be written as $\Delta_{\lambda}=D_{\lambda}^{*} D_{\lambda}$, where $D_{\lambda}=x^{\lambda} \frac{d}{d x} x^{-\lambda}$ and $D_{\lambda}^{*}=$ $-x^{-\lambda} \frac{d}{d x} x^{\lambda}$ is the adjoint operator of $D_{\lambda}$. $D_{\lambda}$ is used to define the Riesz transform in the Bessel setting (see [2]). We consider the operator $\mathcal{G}_{\mathbb{B}}^{\lambda}$ acting on $\mathbb{B}$-valued functions by

$$
\mathcal{G}_{\mathbb{B}}^{\lambda}(f)(t, x)=t D_{\lambda}^{*} P_{t}^{\lambda+1}(f)(x), \quad t, x \in(0, \infty) .
$$

This operator will be useful to get characterizations of UMD Banach spaces.

Theorem 1.2. Let $\lambda \geq 1$. Assume that $\mathbb{B}$ is a UMD Banach space. Then, the operator $\mathcal{G}_{\mathbb{B}}^{\lambda}$ is bounded from $H_{\mathrm{o}}^{1}((0, \infty), \mathbb{B})$ into $H_{\mathrm{o}}^{1}((0, \infty), \gamma(H, \mathbb{B}))$ and from $B M O_{\mathrm{o}}((0, \infty), \mathbb{B})$ into $B M O_{\mathrm{o}}((0, \infty), \gamma(H, \mathbb{B}))$.

We now establish new characterizations of the UMD Banach spaces by using our square functions.

Theorem 1.3. Let $\mathbb{B}$ be a Banach space and $\lambda \geq 1$. The following assertions are equivalent.

(i) $\mathbb{B}$ is UMD.

(ii) There exists $C>0$ such that, for every $f \in E((0, \infty)) \otimes \mathbb{B}$,

$$
\|f\|_{E((0, \infty), \mathbb{B})} \leq C\left\|G_{\mathbb{B}}^{\lambda, 1}(f)\right\|_{E((0, \infty), \gamma(H, \mathbb{B}))}
$$

and

$$
\left\|\mathcal{G}_{\mathbb{B}}^{\lambda}(f)\right\|_{E((0, \infty), \gamma(H, \mathbb{B}))} \leq C\|f\|_{E((0, \infty), \mathbb{B})},
$$

where $E$ denotes $H_{\mathrm{o}}^{1}$ or $B M O_{\mathrm{o}}$.

(iii) For a certain (equivalently, for every) $\beta>0$ there exists $C>0$ such that

$\frac{1}{C}\|f\|_{E((0, \infty), \mathbb{B})} \leq\left\|G_{\mathbb{B}}^{\lambda, \delta}(f)\right\|_{E((0, \infty), \gamma(H, \mathbb{B}))} \leq C\|f\|_{E((0, \infty), \mathbb{B})}, \quad f \in E((0, \infty), \mathbb{B})$,

for $\delta=\beta$ and $\delta=\beta+1$, where $E$ represents $H_{\mathrm{o}}^{1}$ or $B M O_{\mathrm{o}}$.

In the following sections we present proofs of Theorems $1.1,1.2$ and 1.3

Throughout this paper by $c$ and $C$ we represent positive constants not necessarily the same in each occurrence.

\section{Proof of Theorem 1.1}

2.1. In this part we prove that the operator $G_{\mathbb{B}}^{\lambda, \beta}$ is bounded from $H_{\mathrm{o}}^{1}((0, \infty), \mathbb{B})$ into $H_{\mathrm{o}}^{1}((0, \infty), \gamma(H, \mathbb{B}))$.

As in [23] we say that a strongly measurable function $a:(0, \infty) \longrightarrow \mathbb{B}$ is a $q$-atom, where $1<q \leq \infty$, when one of the following two conditions is satisfied:

(Ai) $a=\frac{b}{\delta} \chi_{(0, \delta)}$, where $\delta>0$ and $b \in \mathbb{B}$, being $\|b\|_{\mathbb{B}}=1$

(Aii) there exists an interval $I \subset(0, \infty)$ such that supp $a \subset I,\|a\|_{L^{q}((0, \infty), \mathbb{B})} \leq|I|^{1 / q-1}$ and $\int_{I} a(x) d x=0$.

The arguments presented in the proof of [11, Proposition 3.7] and [23, Theorem 2.1] allow us to get the following. 
Proposition 2.1. Let $X$ be a Banach space, $\lambda>0$ and $1<q<\infty$. Suppose that $f:(0, \infty) \longrightarrow$ $X$ is a strongly measurable function. The following assertions are equivalent:

(i) $f \in H^{1}\left(\Delta_{\lambda}, X\right)$.

(ii) $f \in H_{\mathrm{o}}^{1}((0, \infty), X)$.

(iii) For every $j \in \mathbb{N}$, there exist a q-atom $a_{j}$ and $\lambda_{j} \in \mathbb{C}$ such that $\sum_{j \in \mathbb{N}}\left|\lambda_{j}\right|<\infty$ and $f=\sum_{j \in \mathbb{N}} \lambda_{j} a_{j}$ in $L^{1}((0, \infty), X)$.

Moreover, in this case, we have that

$$
\left\|P_{*}^{\lambda}(f)\right\|_{L^{1}((0, \infty), X)} \sim\|f\|_{H_{\mathrm{o}}^{1}((0, \infty), X)}=\left\|f_{o}\right\|_{H^{1}(\mathbb{R}, X)} \sim \inf \sum_{j \in \mathbb{N}}\left|\lambda_{j}\right|,
$$

where the infimum is taken over all the complex sequences $\left\{\lambda_{j}\right\}_{j \in \mathbb{N}}$ such that $\sum_{j \in \mathbb{N}}\left|\lambda_{j}\right|<\infty$ and, for a certain sequence of q-atoms $\left\{a_{j}\right\}_{j \in \mathbb{N}}, f=\sum_{j \in \mathbb{N}} \lambda_{j} a_{j}$. Here $f_{\mathrm{o}}$ denotes the odd extesion to $\mathbb{R}$ of $f$.

In the sequel we write $H_{\mathrm{o}}^{1}((0, \infty), \mathbb{B})$ to refer us $H^{1}\left(\Delta_{\lambda}, \mathbb{B}\right), \lambda>0$, and we denote by $H^{1}((0, \infty), \mathbb{B})$ the classical $\mathbb{B}$-valued Hardy space on $(0, \infty)$, that is, a function $f \in L^{1}((0, \infty), \mathbb{B})$ is in $H^{1}((0, \infty), \mathbb{B})$ if, and only if, $f=\sum_{j \in \mathbb{N}} \lambda_{j} a_{j}$, where $\lambda_{j} \in \mathbb{C}, j \in \mathbb{N}$, being $\sum_{j \in \mathbb{N}}\left|\lambda_{j}\right|<\infty$, and for some $q \in(1, \infty], a_{j}$ satisfies the condition $($ Aii $)$, for every $j \in \mathbb{N}$.

Our objective is to see that, for every $f \in H_{\mathrm{o}}^{1}((0, \infty), \mathbb{B}), G_{\mathbb{B}}^{\lambda, \beta}(f) \in H_{\mathrm{o}}^{1}((0, \infty), \gamma(H, \mathbb{B}))$, and

$$
\left\|G_{\mathbb{B}}^{\lambda, \beta}(f)\right\|_{H_{\mathrm{o}}^{1}((0, \infty), \gamma(H, \mathbb{B}))} \leq C\|f\|_{H_{\mathrm{o}}^{1}((0, \infty), \mathbb{B})},
$$

where $C>0$ does not depend on $f$.

By taking into account Proposition 2.1, in order to show this, we can see that $G_{\mathbb{B}}^{\lambda, \beta}$ is bounded from $H_{\mathrm{o}}^{1}((0, \infty), \mathbb{B})$ into $L^{1}((0, \infty), \gamma(H, \mathbb{B}))$ (Lemma 2.2 and that $P_{*}^{\lambda} \circ G_{\mathbb{B}}^{\lambda, \beta}$ is bounded from $H_{\mathrm{o}}^{1}((0, \infty), \mathbb{B})$ into $L^{1}(0, \infty)$ (Lemma 2.3 ). We first need to establish the following boundedness property for $G_{\mathbb{B}}^{\lambda, \beta}$.

Lemma 2.1. Let $\mathbb{B}$ be a UMD Banach space, $\beta>0$ and $\lambda \geq 1$. The operator $G_{\mathbb{B}}^{\lambda, \beta}$ is bounded from $L^{1}((0, \infty), \mathbb{B})$ into $L^{1, \infty}((0, \infty), \gamma(H, \mathbb{B}))$ and from $H^{1}((0, \infty), \mathbb{B})$ into $L^{1}((0, \infty), \gamma(H, \mathbb{B}))$.

Proof. Let us define

$$
K_{\lambda}^{\beta}(t ; x, y)=t^{\beta} \partial_{t}^{\beta} P_{t}^{\lambda}(x, y), \quad t, x, y \in(0, \infty),
$$

and consider $m \in \mathbb{N}$ such that $m-1 \leq \beta<m$.

According to [24, (4.6)], for every $k \in \mathbb{N}, \theta \in(0, \pi)$ and $t, s, x, y \in(0, \infty)$, we can write $\partial_{t}^{k}\left[\frac{t+s}{\left[(t+s)^{2}+(x-y)^{2}+2 x y(1-\cos \theta)\right]^{\lambda+1}}\right]=-\frac{1}{2 \lambda} \partial_{t}^{k+1}\left[\frac{1}{\left[(t+s)^{2}+(x-y)^{2}+2 x y(1-\cos \theta)\right]^{\lambda}}\right]$ (6)

$$
=-\frac{1}{2} \sum_{\ell \in \mathbb{N}, 0 \leq \ell \leq(k+1) / 2}(-1)^{k+1-\ell} E_{k+1, \ell}(t+s)^{k+1-2 \ell} \frac{(\lambda+1)(\lambda+2) \cdots(\lambda+k-\ell)}{\left[(t+s)^{2}+(x-y)^{2}+2 x y(1-\cos \theta)\right]^{\lambda+1+k-\ell}},
$$

where $E_{k+1, \ell}=2^{k+1-2 \ell}(k+1) ! /(\ell !(k+1-2 \ell) !), \ell \in \mathbb{N}, 0 \leq \ell \leq(k+1) / 2$.

Then, for each $k \in \mathbb{N}, \theta \in(0, \pi)$ and $t, s, x, y \in(0, \infty)$, $\left|\partial_{t}^{k}\left[\frac{t+s}{\left[(t+s)^{2}+(x-y)^{2}+2 x y(1-\cos \theta)\right]^{\lambda+1}}\right]\right| \leq \frac{C}{(t+s+|x-y|+\sqrt{2 x y(1-\cos \theta)})^{2 \lambda+1+k}}$.

It follows that, for $k \in \mathbb{N}$ and $t, s, x, y \in(0, \infty)$,

$$
\partial_{t}^{k} P_{t+s}^{\lambda}(x, y)=\frac{2 \lambda(x y)^{\lambda}}{\pi} \int_{0}^{\pi}(\sin \theta)^{2 \lambda-1} \partial_{t}^{k}\left[\frac{t+s}{\left[(t+s)^{2}+(x-y)^{2}+2 x y(1-\cos \theta)\right]^{\lambda+1}}\right] d \theta .
$$


Then, for $k \in \mathbb{N}$, we have that

$$
\left|\partial_{t}^{k} P_{t+s}^{\lambda}(x, y)\right| \leq C \frac{(x y)^{\lambda}}{(t+s+|x-y|)^{2 \lambda+1+k}}, \quad t, s, x, y \in(0, \infty),
$$

and also

$$
\begin{aligned}
\left|\partial_{t}^{k} P_{t+s}^{\lambda}(x, y)\right| & \leq C(x y)^{\lambda}\left(\int_{0}^{\pi / 2} \frac{\theta^{2 \lambda-1}}{(t+s+|x-y|+\sqrt{x y} \theta)^{2 \lambda+1+k}} d \theta+\int_{\pi / 2}^{\pi} \frac{(\sin \theta)^{2 \lambda-1}}{(t+s+|x-y|+\sqrt{x y})^{2 \lambda+1+k}} d \theta\right) \\
& \leq C\left(\int_{0}^{\pi \sqrt{x y} / 2} \frac{u^{2 \lambda-1}}{(t+s+|x-y|+u)^{2 \lambda+1+k}} d u+\frac{(x y)^{\lambda}}{(t+s+|x-y|+\sqrt{x y})^{2 \lambda+1+k}}\right)
\end{aligned}
$$

$$
\leq C \frac{1}{(t+s+|x-y|)^{k+1}}, \quad t, s, x, y \in(0, \infty) .
$$

Let $g \in S_{\lambda}(0, \infty)$. We can write

$$
t^{\beta} \partial_{t}^{\beta} P_{t}^{\lambda}(g)(x)=\int_{0}^{\infty} K_{\lambda}^{\beta}(t ; x, y) g(y) d y, \quad t, x \in(0, \infty) .
$$

Indeed, by (7) we can differentiate under the integral sign and write

$$
\partial_{t}^{m} P_{t+s}^{\lambda}(g)(x)=\int_{0}^{\infty} \partial_{t}^{m} P_{t+s}^{\lambda}(x, y) g(y) d y, \quad t, s, x \in(0, \infty),
$$

and, again by (7),

$$
\int_{0}^{\infty} s^{m-\beta-1} \int_{0}^{\infty}\left|\partial_{t}^{m} P_{t+s}^{\lambda}(x, y)\right||g(y)| d y d s \leq C x^{\lambda} \int_{0}^{\infty} \frac{s^{m-\beta-1}}{(t+s)^{2 \lambda+1+m}} d s<\infty, \quad t, x \in(0, \infty) .
$$

Hence,

$$
\begin{aligned}
\partial_{t}^{\beta} P_{t}^{\lambda}(g)(x) & =\frac{e^{-i \pi(m-\beta)}}{\Gamma(m-\beta)} \int_{0}^{\infty} \partial_{t}^{m} P_{t+s}^{\lambda}(g)(x) s^{m-\beta-1} d s \\
& =\int_{0}^{\infty} g(y) \frac{e^{-i \pi(m-\beta)}}{\Gamma(m-\beta)} \int_{0}^{\infty} \partial_{t}^{m} P_{t+s}^{\lambda}(x, y) s^{m-\beta-1} d s d y \\
& =\int_{0}^{\infty} g(y) \partial_{t}^{\beta} P_{t}^{\lambda}(x, y) d y, \quad t, x \in(0, \infty)
\end{aligned}
$$

and (9) is proved.

On the other hand, by using Minkowski's inequality and (8) we deduce that

$$
\begin{aligned}
\left\|K_{\lambda}^{\beta}(\cdot ; x, y)\right\|_{H} & \leq C \int_{0}^{\infty} s^{m-\beta-1}\left(\int_{0}^{\infty}\left|t^{\beta} \partial_{t}^{m} P_{t+s}^{\lambda}(x, y)\right|^{2} \frac{d t}{t}\right)^{1 / 2} d s \\
& \leq C \int_{0}^{\infty} s^{m-\beta-1}\left(\int_{0}^{\infty} \frac{t^{2 \beta-1}}{(t+s+|x-y|)^{2 m+2}} d t\right)^{1 / 2} d s \\
& =C \int_{0}^{\infty} \frac{s^{m-\beta-1}}{(s+|x-y|)^{m-\beta+1}} d s=\frac{C}{|x-y|}, \quad x, y \in(0, \infty), x \neq y .
\end{aligned}
$$

From we infer that the integral in 9 converges as a $H$-Bochner integral, provided that $x \notin \operatorname{supp} g$. We define

$$
F(x)=\int_{0}^{\infty} K_{\lambda}^{\beta}(\cdot ; x, y) g(y) d y, \quad x \notin \operatorname{supp} g,
$$

where the integral is understood in the $H$-Bochner sense. 
Let $h \in H$. According to the properties of the Bochner integrals and Fubini's theorem we get

$$
\begin{aligned}
\int_{0}^{\infty} h(t) F(x)(t) \frac{d t}{t} & =\int_{0}^{\infty} g(y) \int_{0}^{\infty} K_{\lambda}^{\beta}(t ; x, y) h(t) \frac{d t}{t} d y \\
& =\int_{0}^{\infty} h(t) \int_{0}^{\infty} K_{\lambda}^{\beta}(t ; x, y) g(y) d y \frac{d t}{t}, \quad x \notin \operatorname{supp} g .
\end{aligned}
$$

Then, it follows that

$$
G_{\mathbb{C}}^{\lambda, \beta}(g)(\cdot, x)=\int_{0}^{\infty} K_{\lambda}^{\beta}(\cdot ; x, y) g(y) d y, \quad x \notin \operatorname{supp} g,
$$

where the integral is understood in the $H$-Bochner sense.

Suppose now that $g \in S_{\lambda}(0, \infty) \otimes \mathbb{B}$, that is, $g=\sum_{i=1}^{n} b_{i} g_{i}$, where $b_{i} \in \mathbb{B}$ and $g_{i} \in S_{\lambda}(0, \infty)$, $i=1, \ldots, n \in \mathbb{N}$. Since $\gamma(H, \mathbb{C})=H$, we have that $K_{\lambda}^{\beta}(\cdot ; x, y) g(y) \in \gamma(H, \mathbb{B}), x, y \in(0, \infty)$, $x \neq y$, and

$$
\left\|K_{\lambda}^{\beta}(\cdot ; x, y) g(y)\right\|_{\gamma(H, \mathbb{B})} \leq C\left\|K_{\lambda}^{\beta}(\cdot ; x, y)\right\|_{H}\|g(y)\|_{\mathbb{B}} \leq C \frac{\|g(y)\|_{\mathbb{B}}}{|x-y|}, \quad x, y \in(0, \infty), x \neq y .
$$

Also,

$$
\int_{0}^{\infty} K_{\lambda}^{\beta}(\cdot ; x, y) g(y) d y=\sum_{i=1}^{n} b_{i} \int_{0}^{\infty} K_{\lambda}^{\beta}(\cdot ; x, y) g_{i}(y) d y, \quad x \notin \operatorname{supp} g,
$$

where the integral in the left hand side is understood in the $\gamma(H, \mathbb{B})$-Bochner sense and the ones in the right hand side are understood in the $H$-Bochner sense. Hence, we obtain

$$
G_{\mathbb{B}}^{\lambda, \beta}(g)(\cdot, x)=\int_{0}^{\infty} K_{\lambda}^{\beta}(\cdot ; x, y) g(y) d y, \quad x \notin \operatorname{supp} g,
$$

as elements in $\gamma(H, \mathbb{B})$, where the integral is understood in the $\gamma(H, \mathbb{B})$-Bochner sense.

On the other hand, we have that, for every $t, x, y \in(0, \infty)$, and $x \neq y$,

$$
\begin{aligned}
& \partial_{x} K_{\lambda}^{\beta}(t ; x, y)=\frac{t^{\beta} e^{-i \pi(m-\beta)}}{\Gamma(m-\beta)} \int_{0}^{\infty} \partial_{x} \partial_{t}^{m} P_{t+s}^{\lambda}(x, y) s^{m-\beta-1} d s \\
& \quad=\frac{2 \lambda t^{\beta} e^{-i \pi(m-\beta)}}{\pi \Gamma(m-\beta)} \int_{0}^{\infty} s^{m-\beta-1} \int_{0}^{\pi}(\sin \theta)^{2 \lambda-1} \partial_{x} \partial_{t}^{m}\left[\frac{(x y)^{\lambda}(t+s)}{\left[(t+s)^{2}+(x-y)^{2}+2 x y(1-\cos \theta)\right]^{\lambda+1}}\right] d \theta d s .
\end{aligned}
$$

Derivations under the integral signs can be justified as above. By making a derivative with respect to $x$ in (6) for $k=m$, we get

$$
\begin{aligned}
\left|\partial_{x} \partial_{t}^{m} P_{t+s}^{\lambda}(x, y)\right| \leq C\left\{(x y)^{\lambda} \int_{0}^{\pi} \frac{(\sin \theta)^{2 \lambda-1}(|x-y|+y(1-\cos \theta))}{(t+s+|x-y|+\sqrt{2 x y(1-\cos \theta)})^{2 \lambda+m+3}} d \theta\right. \\
\left.\quad+x^{\lambda-1} y^{\lambda} \int_{0}^{\pi} \frac{(\sin \theta)^{2 \lambda-1}}{(t+s+|x-y|+\sqrt{2 x y(1-\cos \theta)})^{2 \lambda+m+1}} d \theta\right\} \\
\leq C\left\{(x y)^{\lambda} \int_{0}^{\pi} \frac{(\sin \theta)^{2 \lambda-1}}{(t+s+|x-y|+\sqrt{2 x y(1-\cos \theta)})^{2 \lambda+m+2}} d \theta\right. \\
\left.\quad+x^{\lambda-1} y^{\lambda} \int_{0}^{\pi} \frac{(\sin \theta)^{2 \lambda-1}}{(t+s+|x-y|+\sqrt{2 x y(1-\cos \theta)})^{2 \lambda+m+1}} d \theta\right\} \\
=C\left\{I_{1}(t, s ; x, y)+I_{2}(t, s ; x, y)\right\}, \quad t, s, x, y \in(0, \infty) .
\end{aligned}
$$

By proceeding as in the proof of $(8)$ for $k=m+1$ we obtain

$$
I_{1}(t, s ; x, y) \leq \frac{C}{(t+s+|x-y|)^{m+2}}, \quad t, s, x, y \in(0, \infty) .
$$


Since $\lambda \geq 1$, we also have that

$$
\begin{aligned}
I_{2}(t, s ; x, y) & \leq C y\left\{\int_{0}^{\pi / 2} \frac{\theta^{2 \lambda-1}(x y)^{\lambda-1}}{(t+s+|x-y|+\sqrt{x y} \theta)^{2 \lambda+m+1}} d \theta+\frac{(x y)^{\lambda-1}}{(t+s+|x-y|+\sqrt{x y})^{2 \lambda+m+1}}\right\} \\
& \leq C y\left\{\int_{0}^{\pi / 2} \frac{\theta}{(t+s+|x-y|+\sqrt{x y} \theta)^{m+3}} d \theta+\frac{1}{(t+s+|x-y|+\sqrt{x y})^{m+3}}\right\} \\
& \leq C\left\{\begin{array}{l}
\int_{0}^{\pi / 2} \frac{\theta y}{(t+s+|x-y|+y \theta)^{m+3}} d \theta+\frac{1}{(t+s+|x-y|)^{m+2}}, \quad 0<y \leq 2 x, x \neq y, \\
\frac{y}{(t+s+|x-y|)^{m+3}}, \quad 0<2 x \leq y, \\
(14) \quad
\end{array}\right. \\
& \leq C \frac{1}{(t+s+|x-y|)^{m+2}}, \quad t, s, x, y \in(0, \infty) .
\end{aligned}
$$

Hence, by Minkowski's inequality we get

$$
\begin{aligned}
\left\|\partial_{x} K_{\lambda}^{\beta}(\cdot ; x, y)\right\|_{H} & \leq C \int_{0}^{\infty} s^{m-\beta-1}\left(\int_{0}^{\infty} t^{2 \beta-1}\left|\partial_{x} \partial_{t}^{m} P_{t+s}^{\lambda}(x, y)\right|^{2} d t\right)^{1 / 2} d s \\
& \leq C \int_{0}^{\infty} s^{m-\beta-1}\left(\int_{0}^{\infty} \frac{t^{2 \beta-1}}{(t+s+|x-y|)^{2 m+4}} d t\right)^{1 / 2} d s=\frac{C}{|x-y|^{2}}, \quad x, y \in(0, \infty), x \neq y
\end{aligned}
$$

We conclude that

$$
\left\|\partial_{x} K_{\lambda}^{\beta}(\cdot ; x, y)\right\|_{H} \leq \frac{C}{|x-y|^{2}}, \quad x, y \in(0, \infty), x \neq y
$$

By taking into account symmetries we also get

$$
\left\|\partial_{y} K_{\lambda}^{\beta}(\cdot, x, y)\right\|_{H} \leq \frac{C}{|x-y|^{2}}, \quad x, y \in(0, \infty), x \neq y
$$

Fix now $x, y \in(0, \infty), x \neq y$. We define the operator $L(x, y)$ by

$$
\begin{aligned}
L(x, y): \mathbb{B} & \longrightarrow \gamma(H, \mathbb{B}) \\
b & \longmapsto L(x, y) b=K_{\lambda}^{\beta}(\cdot ; x, y) b .
\end{aligned}
$$

If $\left\{h_{j}\right\}_{j \in \mathbb{N}}$ is an orthonormal basis in $H$, since $\gamma(H, \mathbb{C})=H$, we deduce from 10 ,

$$
\begin{aligned}
\|L(x, y) b\|_{\gamma(H, \mathbb{B})} & =\left\|K_{\lambda}^{\beta}(\cdot, x, y) b\right\|_{\gamma(H, \mathbb{B})}=\left(\mathbb{E}\left\|\sum_{j=1}^{\infty} \gamma_{j} \int_{0}^{\infty} K_{\lambda}^{\beta}(t ; x, y) h_{j}(t) \frac{d t}{t} b\right\|_{\mathbb{B}}^{2}\right)^{1 / 2} \\
& =\|b\|_{\mathbb{B}}\left(\mathbb{E}\left|\sum_{j=1}^{\infty} \gamma_{j} \int_{0}^{\infty} K_{\lambda}^{\beta}(t ; x, y) h_{j}(t) \frac{d t}{t}\right|^{2}\right)^{1 / 2} \leq C\|b\|_{\mathbb{B}}\left\|K_{\lambda}^{\beta}(\cdot ; x, y)\right\|_{H} \\
& \leq C \frac{\|b\|_{\mathbb{B}}}{|x-y|}, \quad b \in \mathbb{B} .
\end{aligned}
$$

Hence,

$$
\|L(x, y)\|_{\mathbb{B} \rightarrow \gamma(H, \mathbb{B})} \leq \frac{C}{|x-y|} .
$$

In a similar way, by identifying $\partial_{x} K_{\lambda}^{\beta}(\cdot ; x, y)$ and $\partial_{y} K_{\lambda}^{\beta}(\cdot ; x, y)$ with the corresponding operators in $L(\mathbb{B}, \gamma(H, \mathbb{B}))$, the space of linear and bounded operators from $\mathbb{B}$ into $\gamma(H, \mathbb{B})$, and by using (15) and (16), we can get that

$$
\left\|\partial_{x} K_{\lambda}^{\beta}(\cdot ; x, y)\right\|_{\mathbb{B} \rightarrow \gamma(H, \mathbb{B})} \leq \frac{C}{|x-y|^{2}},
$$


and

$$
\left\|\partial_{y} K_{\lambda}^{\beta}(\cdot ; x, y)\right\|_{\mathbb{B} \rightarrow \gamma(H, \mathbb{B})} \leq \frac{C}{|x-y|^{2}}
$$

By Theorem $\mathbb{B}, G_{\mathbb{B}}^{\lambda, \beta}$ is a bounded operator from $L^{p}((0, \infty), \mathbb{B})$ into $L^{p}((0, \infty), \gamma(H, \mathbb{B}))$, for every $1<p<\infty$. Then, from (11), (17), 18) and 119 we conclude that the operator $G_{\mathbb{B}}^{\lambda, \beta}$ is a $(\mathbb{B}, \gamma(H, \mathbb{B}))$-Calderón-Zygmund operator. Hence, $G_{\mathbb{B}}^{\lambda, \beta}$ can be extended from $S_{\lambda}(0, \infty) \otimes \mathbb{B}$ to $L^{1}((0, \infty), \mathbb{B})$ as a bounded operator from $L^{1}((0, \infty), \mathbb{B})$ into $L^{1, \infty}((0, \infty), \gamma(H, \mathbb{B}))$ and to $H^{1}((0, \infty), \mathbb{B})$ as a bounded operator from $H^{1}((0, \infty), \mathbb{B})$ into $L^{1}((0, \infty), \gamma(H, \mathbb{B}))$. We denote by $\widetilde{G}_{\mathbb{B}}^{\lambda, \beta}$ the extension of $G_{\mathbb{B}}^{\lambda, \beta}$ to $L^{1}((0, \infty), \mathbb{B})$ as a bounded operator from $L^{1}((0, \infty), \mathbb{B})$ into $L^{1, \infty}((0, \infty), \gamma(H, \mathbb{B}))$.

We now prove that

$$
\widetilde{G}_{\mathbb{B}}^{\lambda, \beta}(g)(x)=G_{\mathbb{B}}^{\lambda, \beta}(g)(\cdot, x), \quad \text { a.e. } x \in(0, \infty),
$$

as elements of $\gamma(H, \mathbb{B})$, for every $g \in L^{1}((0, \infty), \mathbb{B})$.

Let $g \in L^{1}((0, \infty), \mathbb{B})$. We choose a sequence $\left(g_{k}\right)_{k \in \mathbb{N}} \subset S_{\lambda}(0, \infty) \otimes \mathbb{B}$ such that

$$
g_{k} \longrightarrow g, \quad \text { as } k \rightarrow \infty, \text { in } L^{1}((0, \infty), \mathbb{B})
$$

Then,

$$
G_{\mathbb{B}}^{\lambda, \beta}\left(g_{k}\right) \longrightarrow \widetilde{G}_{\mathbb{B}}^{\lambda, \beta}(g), \quad \text { as } k \rightarrow \infty, \text { in } L^{1, \infty}((0, \infty), \gamma(H, \mathbb{B})),
$$

and hence, there exist a set $\Omega \subset(0, \infty)$, being $|(0, \infty) \backslash \Omega|=0$, and an increasing sequence $\left(n_{k}\right)_{k \in \mathbb{N}} \subset \mathbb{N}$ such that, for every $x \in \Omega$,

$$
G_{\mathbb{B}}^{\lambda, \beta}\left(g_{n_{k}}\right)(\cdot, x) \longrightarrow \widetilde{G}_{\mathbb{B}}^{\lambda, \beta}(g)(x), \quad \text { as } k \rightarrow \infty, \text { in } \gamma(H, \mathbb{B}) .
$$

Let $\varepsilon>0$. By proceeding as in the proof of 10 we obtain

$$
\begin{aligned}
\left\|K_{\lambda}^{\beta}(\cdot ; x, y)\right\|_{L^{2}((\varepsilon, \infty), d t / t)} & \leq C \int_{0}^{\infty} s^{m-\beta-1}\left(\int_{\varepsilon}^{\infty} \frac{t^{2 \beta-1}}{(t+s+|x-y|)^{2 m+2}} d t\right)^{1 / 2} d s \\
& =C \int_{0}^{\infty} s^{m-\beta-1}\left(\int_{0}^{\infty} \frac{(t+\varepsilon)^{2 \beta-1}}{(t+\varepsilon+s+|x-y|)^{2 m+2}} d t\right)^{1 / 2} d s \\
& \leq C \int_{0}^{\infty} s^{m-\beta-1}\left(\int_{0}^{\infty} \frac{t^{2 \beta-1}+\varepsilon^{2 \beta-1}}{(t+\varepsilon+s+|x-y|)^{2 m+2}} d t\right)^{1 / 2} d s \\
& \leq C\left(\frac{1}{\varepsilon+|x-y|}+\frac{\varepsilon^{\beta-1 / 2}}{(\varepsilon+|x-y|)^{\beta+1 / 2}}\right) \leq \frac{C}{\varepsilon}, \quad x, y \in(0, \infty) .
\end{aligned}
$$

Then, by using Minkowski's inequality it follows that

$$
\begin{aligned}
\left\|G_{\mathbb{B}}^{\lambda, \beta}\left(g_{n_{k}}\right)(\cdot, x)-G_{\mathbb{B}}^{\lambda, \beta}(g)(\cdot, x)\right\|_{L^{2}((\varepsilon, \infty), d t / t, \mathbb{B})} & \leq C \int_{0}^{\infty}\left\|g(y)-g_{n_{k}}(y)\right\|_{\mathbb{B}}\left\|K_{\lambda}^{\beta}(\cdot ; x, y)\right\|_{L^{2}((\varepsilon, \infty), d t / t)} d y \\
& \leq \frac{C}{\varepsilon}\left\|g-g_{n_{k}}\right\|_{L^{1}((0, \infty), \mathbb{B}), \quad x \in(0, \infty)}
\end{aligned}
$$

that is,

$$
G_{\mathbb{B}}^{\lambda, \beta}\left(g_{n_{k}}\right)(\cdot, x) \longrightarrow G_{\mathbb{B}}^{\lambda, \beta}(g)(\cdot, x), \quad \text { as } k \rightarrow \infty, \text { in } L^{2}((\varepsilon, \infty), d t / t, \mathbb{B}),
$$

uniformly in $x \in(0, \infty)$.

By taking into account that $\gamma(H, \mathbb{B})$ is continuously contained in $L(H, \mathbb{B})$, the space of bounded linear operators from $H$ into $\mathbb{B}$, it follows that, for every $S \in \mathbb{B}^{*}$ and $h \in C_{c}^{\infty}(0, \infty)$, the space 
of smooth functions with compact support on $(0, \infty)$, we have that

$$
\begin{aligned}
\left\langle S, \widetilde{G}_{\mathbb{B}}^{\lambda, \beta}(g)(x)[h]\right\rangle_{\mathbb{B}^{*}, \mathbb{B}} & =\lim _{k \rightarrow \infty}\left\langle S, G_{\mathbb{B}}^{\lambda, \beta}\left(g_{n_{k}}\right)(\cdot, x)[h]\right\rangle_{\mathbb{B}^{*}, \mathbb{B}}=\lim _{k \rightarrow \infty} \int_{0}^{\infty}\left\langle S, G_{\mathbb{B}}^{\lambda, \beta}\left(g_{n_{k}}\right)(t, x)\right\rangle_{\mathbb{B}^{*}, \mathbb{B}} h(t) \frac{d t}{t} \\
& =\int_{0}^{\infty}\left\langle S, G_{\mathbb{B}}^{\lambda, \beta}(g)(t, x)\right\rangle_{\mathbb{B}^{*}, \mathbb{B}} h(t) \frac{d t}{t}, \quad x \in \Omega,
\end{aligned}
$$

and then,

$\left|\int_{0}^{\infty}\left\langle S, G_{\mathbb{B}}^{\lambda, \beta}(g)(t, x)\right\rangle_{\mathbb{B}^{*}, \mathbb{B}} h(t) \frac{d t}{t}\right| \leq\|S\|_{\mathbb{B}^{*}}\left\|\widetilde{G}_{\mathbb{B}}^{\lambda, \beta}(g)(x)[h]\right\|_{\mathbb{B}} \leq\|S\|_{\mathbb{B}^{*}}\left\|\widetilde{G}_{\mathbb{B}}^{\lambda, \beta}(g)(x)\right\|_{H \rightarrow \mathbb{B}}\|h\|_{H}, \quad x \in \Omega$.

Hence, $\left\langle S, G_{\mathbb{B}}^{\lambda, \beta}(g)(\cdot, x)\right\rangle_{\mathbb{B}^{*}, \mathbb{B}} \in H, x \in \Omega$, and

$$
\widetilde{G}_{\mathbb{B}}^{\lambda, \beta}(g)(x)=G_{\mathbb{B}}^{\lambda, \beta}(g)(\cdot, x), \quad x \in \Omega .
$$

We conclude that $G_{\mathbb{B}}^{\lambda, \beta}$ is bounded from $L^{1}((0, \infty), \mathbb{B})$ into $L^{1, \infty}((0, \infty), \gamma(H, \mathbb{B}))$ and from $H^{1}((0, \infty), \mathbb{B})$ into $L^{1}((0, \infty), \gamma(H, \mathbb{B}))$.

Next, we establish the behavior of $G_{\mathbb{B}}^{\lambda, \beta}$ on $H_{\mathrm{o}}^{1}((0, \infty), \mathbb{B})$.

Lemma 2.2. Let $\mathbb{B}$ be a UMD Banach space, $\beta>0$ and $\lambda \geq 1$. The operator $G_{\mathbb{B}}^{\lambda, \beta}$ is bounded from $H_{\mathrm{o}}^{1}((0, \infty), \mathbb{B})$ into $L^{1}((0, \infty), \gamma(H, \mathbb{B}))$.

Proof. Let $f \in H_{\mathrm{o}}^{1}((0, \infty), \mathbb{B})$. By Proposition 2.1 we write $f=\sum_{j \in \mathbb{N}} \lambda_{j} a_{j}$, where $a_{j}$ is a 2-atom and $\lambda_{j} \in \mathbb{C}, j \in \mathbb{N}$, being $\sum_{j \in \mathbb{N}}\left|\lambda_{j}\right|<\infty$. Since the series $\sum_{j \in \mathbb{N}} \lambda_{j} a_{j}$ converges in $L^{1}((0, \infty), \mathbb{B})$ and $G_{\mathbb{B}}^{\lambda, \beta}$ is bounded from $L^{1}((0, \infty), \mathbb{B})$ into $\left.L^{1, \infty}(0, \infty), \gamma(H, \mathbb{B})\right)$ (Lemma 2.1), we have that

$$
G_{\mathbb{B}}^{\lambda, \beta}(f)=\sum_{j \in \mathbb{N}} \lambda_{j} G_{\mathbb{B}}^{\lambda, \beta}\left(a_{j}\right)
$$

where the series converges in $L^{1, \infty}((0, \infty), \gamma(H, \mathbb{B}))$.

If $a$ is a 2-atom satisfying (Aii), since, by Lemma 2.1. $G_{\mathbb{B}}^{\lambda, \beta}$ is a bounded operator from $H^{1}((0, \infty), \mathbb{B})$ into $L^{1}((0, \infty), \gamma(H, \mathbb{B}))$, we get

$$
\left\|G_{\mathbb{B}}^{\lambda, \beta}(a)\right\|_{L^{1}((0, \infty), \gamma(H, \mathbb{B}))} \leq C,
$$

being $C>0$ independent of $a$.

Suppose now that $a=b \chi_{(0, \delta)} / \delta$, for some $\delta>0$ and $b \in \mathbb{B},\|b\|_{\mathbb{B}}=1$. By taking into account that $G_{\mathbb{B}}^{\lambda, \beta}$ is bounded from $L^{2}((0, \infty), \mathbb{B})$ into $L^{2}((0, \infty), \gamma(H, \mathbb{B}))$ (Theorem $\mathbb{B}$, we obtain

$$
\int_{0}^{2 \delta}\left\|G_{\mathbb{B}}^{\lambda, \beta}(a)(\cdot, x)\right\|_{\gamma(H, \mathbb{B})} d x \leq(2 \delta)^{1 / 2}\left\|G_{\mathbb{B}}^{\lambda, \beta}(a)\right\|_{L^{2}((0, \infty), \gamma(H, \mathbb{B}))} \leq C \delta^{1 / 2}\|a\|_{L^{2}((0, \infty), \mathbb{B})} \leq C,
$$

where $C>0$ does not depend on $\delta$ or $b$.

According to 11 , since $\gamma(H, \mathbb{C})=H$, we have that

$$
\left\|G_{\mathbb{B}}^{\lambda, \beta}(a)(\cdot, x)\right\|_{\gamma(H, \mathbb{B})} \leq \frac{1}{\delta} \int_{0}^{\delta}\left\|K_{\lambda}^{\beta}(\cdot ; x, y)\right\|_{H} d y, \quad x \geq 2 \delta .
$$

By proceeding as in (10) and taking into account 77 we can write

$$
\begin{aligned}
& \left\|K_{\lambda}^{\beta}(\cdot ; x, y)\right\|_{H} \leq C(x y)^{\lambda} \int_{0}^{\infty} s^{m-\beta-1}\left(\int_{0}^{\infty} \frac{t^{2 \beta-1}}{(t+s+|x-y|)^{4 \lambda+2 m+2}} d t\right)^{1 / 2} d s \\
& \quad \leq C(x y)^{\lambda} \int_{0}^{\infty} \frac{s^{m-\beta-1}}{(s+|x-y|)^{2 \lambda+1+m-\beta}} d s \leq C \frac{(x y)^{\lambda}}{|x-y|^{2 \lambda+1}}, \quad x, y \in(0, \infty), x \neq y .
\end{aligned}
$$


Hence, it follows that

$$
\left\|G_{\mathbb{B}}^{\lambda, \beta}(a)(\cdot, x)\right\|_{\gamma(H, \mathbb{B})} \leq \frac{C}{\delta} \int_{0}^{\delta} \frac{(x y)^{\lambda}}{|x-y|^{2 \lambda+1}} d y \leq \frac{C}{\delta x^{\lambda+1}} \int_{0}^{\delta} y^{\lambda} d y \leq C \frac{\delta^{\lambda}}{x^{\lambda+1}}, \quad x \geq 2 \delta
$$

and we get

$$
\int_{2 \delta}^{\infty}\left\|G_{\mathbb{B}}^{\lambda, \beta}(a)(\cdot, x)\right\|_{\gamma(H, \mathbb{B})} d x \leq C \int_{2 \delta}^{\infty} \frac{\delta^{\lambda}}{x^{\lambda+1}} d x \leq C
$$

where $C>0$ does not depend on $\delta$ and $b$.

From 22 and 24 we deduce

$$
\left\|G_{\mathbb{B}}^{\lambda, \beta}(a)\right\|_{L^{1}((0, \infty), \gamma(H, \mathbb{B}))} \leq C,
$$

where $C>0$ is independent of $\delta$ and $b$.

By using 20, 21) and 25) we conclude

$$
\left\|G_{\mathbb{B}}^{\lambda, \beta}(f)\right\|_{L^{1}((0, \infty), \gamma(H, \mathbb{B}))} \leq C \sum_{j \in \mathbb{N}}\left|\lambda_{j}\right|
$$

Hence,

$$
\left\|G_{\mathbb{B}}^{\lambda, \beta}(f)\right\|_{L^{1}((0, \infty), \gamma(H, \mathbb{B}))} \leq C\|f\|_{H_{\mathrm{o}}^{1}(\mathbb{R}, \mathbb{B})}
$$

According to [16, Theorem 2.4] the maximal operator $P_{*}^{\lambda}$ given by

$$
P_{*}^{\lambda}(g)(x)=\sup _{s>0}\left\|P_{s}^{\lambda}(g)(x)\right\|_{\gamma(H, \mathbb{B})},
$$

for every $g \in L^{p}((0, \infty), \gamma(H, \mathbb{B})), 1 \leq p \leq \infty$, is bounded from $L^{p}((0, \infty), \gamma(H, \mathbb{B}))$ into $L^{p}(0, \infty)$, for every $1<p<\infty$, and from $L^{1}((0, \infty), \gamma(H, \mathbb{B}))$ into $L^{1, \infty}(0, \infty)$. Then, the operator $P_{*}^{\lambda} \circ G_{\mathbb{B}}^{\lambda, \beta}$ is bounded from $H^{1}((0, \infty), \mathbb{B})$ into $L^{1, \infty}(0, \infty)$.

We now show that $P_{*}^{\lambda} \circ G_{\mathbb{B}}^{\lambda, \beta}$ is a bounded operator from $H_{\mathrm{o}}^{1}((0, \infty), \mathbb{B})$ into $L^{1}(0, \infty)$.

Lemma 2.3. Let $\mathbb{B}$ be a UMD Banach space, $\beta>0$ and $\lambda \geq 1$. We have that $P_{*}^{\lambda} \circ G_{\mathbb{B}}^{\lambda, \beta}$ is a bounded operator from $H_{\mathrm{o}}^{1}((0, \infty), \mathbb{B})$ into $L^{1}(0, \infty)$.

Proof. Note firstly that $P_{*}^{\lambda} \circ G_{\mathbb{B}}^{\lambda, \beta}$ is bounded from $H_{\mathrm{o}}^{1}((0, \infty), \mathbb{B})$ into $L^{1, \infty}(0, \infty)$.

According to [6. Lemma 3.1], we have that, for every $\phi \in S_{\lambda}(0, \infty)$,

$$
h_{\lambda}\left(t^{\beta} \partial_{t}^{\beta} P_{t}^{\lambda}(\phi)\right)=e^{i \beta \pi}(t y)^{\beta} e^{-y t} h_{\lambda}(\phi)(y), \quad t>0 .
$$

Since $h_{\lambda}$ is an isometry in $L^{2}(0, \infty)$, it follows that $t^{\beta} \partial_{t}^{\beta} P_{t}^{\lambda}(\phi) \in L^{2}(0, \infty)$, for every $\phi \in S_{\lambda}(0, \infty)$ and $t>0$, and by [22, $\S 8.5(19)]$, we get

$$
h_{\lambda}\left(P_{s}^{\lambda}\left[t^{\beta} \partial_{t}^{\beta} P_{t}^{\lambda}(\phi)\right]\right)(x)=e^{i \pi \beta}(t x)^{\beta} e^{-x(t+s)} h_{\lambda}(\phi)(x), \quad \phi \in S_{\lambda}(0, \infty) \text { and } t, s, x \in(0, \infty),
$$

and then, for every $\phi \in S_{\lambda}(0, \infty)$ and $t, s, x \in(0, \infty)$,

$$
P_{s}^{\lambda}\left[t^{\beta} \partial_{t}^{\beta} P_{t}^{\lambda}(\phi)\right](x)=t^{\beta} \partial_{t}^{\beta} P_{t+s}^{\lambda}(\phi)(x)=\int_{0}^{\infty} t^{\beta} \partial_{t}^{\beta} P_{t+s}^{\lambda}(x, y) \phi(y) d y .
$$

Also, for every $\phi \in S_{\lambda}(0, \infty) \otimes \mathbb{B}$,

$$
P_{s}^{\lambda}\left(G_{\mathbb{B}}^{\lambda, \beta}(\phi)(t, \cdot)\right)(x)=P_{s}^{\lambda}\left[t^{\beta} \partial_{t}^{\beta} P_{t}^{\lambda}(\phi)\right](x)=t^{\beta} \partial_{t}^{\beta} P_{t+s}^{\lambda}(\phi)(x), \quad t, s, x \in(0, \infty) .
$$

By defining the function

$$
K_{\lambda}^{\beta}(t, s ; x, y)=t^{\beta} \partial_{t}^{\beta} P_{t+s}^{\lambda}(x, y), \quad t, s, x, y \in(0, \infty),
$$


we have that

$$
\left\|K_{\lambda}^{\beta}(\cdot, \cdot ; x, y)\right\|_{L^{\infty}((0, \infty), H)} \leq \frac{C}{|x-y|}, \quad x, y \in(0, \infty), x \neq y .
$$

Indeed, as in the proof of 10, , by using $(8)$ we get

$$
\begin{aligned}
& \left\|K_{\lambda}^{\beta}(\cdot, \cdot ; x, y)\right\|_{L^{\infty}((0, \infty), H)}=\sup _{s>0}\left\|t^{\beta} \partial_{t}^{\beta} P_{t+s}^{\lambda}(x, y)\right\|_{H} \\
& \quad \leq C \sup _{s>0} \int_{0}^{\infty} u^{m-\beta-1}\left(\int_{0}^{\infty} \frac{t^{2 \beta-1}}{(t+s+u+|x-y|)^{2 m+2}} d t\right)^{1 / 2} d u \\
& \quad \leq C \sup _{s>0} \frac{1}{s+|x-y|} \leq \frac{C}{|x-y|}, \quad x, y \in(0, \infty), x \neq y .
\end{aligned}
$$

Also, we get, for every $x, y \in(0, \infty), x \neq y$,

$$
\left\|\partial_{x} K_{\lambda}^{\beta}(\cdot, \cdot ; x, y)\right\|_{L^{\infty}((0, \infty), H)}+\left\|\partial_{y} K_{\lambda}^{\beta}(\cdot, \cdot ; x, y)\right\|_{L^{\infty}((0, \infty), H)} \leq \frac{C}{|x-y|^{2}} .
$$

According to 26 , for every $\phi \in S_{\lambda}(0, \infty)$, the integral

$$
\int_{0}^{\infty} K_{\lambda}^{\beta}(\cdot, \cdot ; x, y) \phi(y) d y
$$

is convergent in the $L^{\infty}((0, \infty), H)$-Bochner sense provided that $x \notin \operatorname{supp} \phi$.

Let $N \in \mathbb{N}$. We define the operator $Q_{\lambda, N}^{\beta}$ as follows:

$$
Q_{\lambda, N}^{\beta}(\phi)(x)=\int_{0}^{\infty} K_{\lambda}^{\beta}(\cdot, \cdot ; x, y) \phi(y) d y, \quad \phi \in S_{\lambda}(0, \infty) \text { and } x \notin \operatorname{supp} \phi,
$$

where the integral is understood in the $C([1 / N, N], H)$-Bochner sense. Here, by $C([1 / N, N], H)$ we denote the space of $H$-valued continuous functions on $[1 / N, N]$.

Let $\phi \in S_{\lambda}(0, \infty)$. We have that

$$
\left[Q_{\lambda, N}^{\beta}(\phi)(x)\right](s)=\int_{0}^{\infty} K_{\lambda}^{\beta}(\cdot, s ; x, y) \phi(y) d y, \quad x \notin \operatorname{supp} \phi \text { and } s \in[1 / N, N],
$$

where the integral is understood in the $H$-Bochner sense and the equality is understood in $H$.

Moreover, according to some properties of Bochner integration and by applying Fubini's theorem, we get

$$
\begin{aligned}
\int_{0}^{\infty} & {\left[\left[Q_{\lambda, N}^{\beta}(\phi)(x)\right](s)\right](t) h(t) \frac{d t}{t}=\int_{0}^{\infty} \phi(y) \int_{0}^{\infty} K_{\lambda}^{\beta}(t, s ; x, y) h(t) \frac{d t}{t} d y } \\
& =\int_{0}^{\infty} h(t) \int_{0}^{\infty} K_{\lambda}^{\beta}(t, s ; x, y) \phi(y) d y \frac{d t}{t}, \quad x \notin \operatorname{supp} \phi \text { and } s \in[1 / N, N] .
\end{aligned}
$$

Then,

$$
\left[\left[Q_{\lambda, N}^{\beta}(\phi)(x)\right](s)\right](t)=\int_{0}^{\infty} K_{\lambda}^{\beta}(t, s ; x, y) \phi(y) d y, \quad x \notin \operatorname{supp} \phi \text { and } s \in[1 / N, N],
$$

as elements of $H$.

We define $Q_{\lambda, N}^{\beta}$ on $S_{\lambda}(0, \infty) \otimes \mathbb{B}$ in the natural way. For every $\phi \in S_{\lambda}(0, \infty) \otimes \mathbb{B}$ we have that

$$
\left[\left[Q_{\lambda, N}^{\beta}(\phi)(x)\right](s)\right](t)=P_{s}^{\lambda}\left(G_{\mathbb{B}}^{\lambda, \beta}(\phi)(t, \cdot)\right)(x), \quad x \notin \operatorname{supp} \phi \text { and } s \in[1 / N, N]
$$

in the sense of equality in $\gamma(H, \mathbb{B})$.

Since $P_{*}^{\lambda}$ is bounded from $L^{2}((0, \infty), \gamma(H, \mathbb{B}))$ into $L^{2}(0, \infty)$ and $G_{\mathbb{B}}^{\lambda, \beta}$ is bounded from $L^{2}((0, \infty), \mathbb{B})$ into $L^{2}\left((0, \infty), \gamma(H, \mathbb{B})\right.$ ) (Theorem $\mathbb{B}$, the operator $P_{*}^{\lambda} \circ G_{\mathbb{B}}^{\lambda, \beta}$ is bounded from $L^{2}((0, \infty), \mathbb{B})$ into $L^{2}(0, \infty)$. Hence, the operator

$$
Z_{\lambda, N}^{\beta}(f)(t, s ; x)=P_{s}^{\lambda}\left(G_{\mathbb{B}}^{\lambda, \beta}(f)(t, \cdot)\right)(x), \quad t, x \in(0, \infty), s \in[1 / N, N],
$$


is bounded from $L^{2}((0, \infty), \mathbb{B})$ into $L^{2}((0, \infty), C([1 / N, N], \gamma(H, \mathbb{B})))$. Moreover,

$$
\sup _{M \in \mathbb{N}}\left\|Z_{\lambda, M}^{\beta}\right\|_{L^{2}((0, \infty), \mathbb{B}) \rightarrow L^{2}((0, \infty), C([1 / M, M], \gamma(H, \mathbb{B})))}<\infty .
$$

By taking into account (26), (27) and (28) and by using vector valued Calderón-Zygmund theory we conclude that the operator $Z_{\lambda, N}^{\beta}$ can be extended to $L^{1}((0, \infty), \mathbb{B})$ as a bounded operator from $L^{1}((0, \infty), \mathbb{B})$ into $L^{1, \infty}((0, \infty), C([1 / N, N], \gamma(H, \mathbb{B})))$ and to $H^{1}((0, \infty), \mathbb{B})$ as a bounded operator from $H^{1}((0, \infty), \mathbb{B})$ into $L^{1}((0, \infty), C([1 / N, N], \gamma(H, \mathbb{B})))$. We denote by $\widetilde{Z}_{\lambda, N}^{\beta}$ to this extension of $Z_{\lambda, N}^{\beta}$ to $L^{1}((0, \infty), \mathbb{B})$. It has that

$$
\sup _{M \in \mathbb{N}}\left\|\widetilde{Z}_{\lambda, M}^{\beta}\right\|_{L^{1}((0, \infty), \mathbb{B}) \rightarrow L^{1, \infty}((0, \infty), C([1 / M, M], \gamma(M, \mathbb{B})))}<\infty,
$$

and

$$
\sup _{M \in \mathbb{N}}\left\|\widetilde{Z}_{\lambda, M}^{\beta}\right\|_{H^{1}((0, \infty), \mathbb{B}) \rightarrow L^{1}((0, \infty), C([1 / M, M], \gamma(H, \mathbb{B})))}<\infty .
$$

Our objective now is to show that

$$
\widetilde{Z}_{\lambda, N}^{\beta}(f)=P_{s}^{\lambda}\left(G_{\mathbb{B}}^{\lambda, \beta}(f)\right), \quad f \in L^{1}((0, \infty), \mathbb{B}),
$$

as elements in $L^{1, \infty}((0, \infty), C([1 / N, N], \gamma(H, \mathbb{B})))$.

Let $f \in L^{1}((0, \infty), \mathbb{B})$. We choose a sequence $\left(f_{k}\right)_{k \in \mathbb{N}} \subset S_{\lambda}(0, \infty) \otimes \mathbb{B}$ such that

$$
f_{k} \longrightarrow f, \quad \text { as } k \rightarrow \infty, \text { in } L^{1}((0, \infty), \mathbb{B}) .
$$

Then,

$$
Z_{\lambda, N}^{\beta}\left(f_{k}\right) \longrightarrow \widetilde{Z}_{\lambda, N}^{\beta}(f), \quad \text { as } k \rightarrow \infty, \text { in } L^{1, \infty}((0, \infty), C([1 / N, N], \gamma(H, \mathbb{B}))) .
$$

It is not hard to see that, for every $t, x \in(0, \infty)$ and $s \in[1 / N, N]$,

$$
Z_{\lambda, N}^{\beta}(f)(t, s ; x)=\int_{0}^{\infty} K_{\lambda}^{\beta}(t, s ; x, y) f(y) d y=G_{\mathbb{B}}^{\lambda, \beta}\left(P_{s}^{\lambda}(f)\right)(t, x) .
$$

We know that, for every $s \in(0, \infty), P_{s}^{\lambda}$ is a bounded operator from $L^{1}((0, \infty), \mathbb{B})$ into itself, and that the operator $G_{\mathbb{B}}^{\lambda, \beta}$ is bounded from $L^{1}((0, \infty), \mathbb{B})$ into $L^{1, \infty}((0, \infty), \gamma(H, \mathbb{B}))$ (Lemma 2.1). Then, it follows that, for every $s \in[1 / N, N]$,

$$
Z_{\lambda, N}^{\beta}\left(f_{k}\right)(\cdot, s ; \cdot) \longrightarrow Z_{\lambda, N}^{\beta}(f)(\cdot, s ; \cdot), \quad \text { as } k \rightarrow \infty, \text { in } L^{1, \infty}((0, \infty), \gamma(H, \mathbb{B})) .
$$

Hence, we can find an increasing sequence $\left(n_{k}\right)_{k \in \mathbb{N}} \subset \mathbb{N}$ and a subset $\Omega$ of $(0, \infty)$ such that $|(0, \infty) \backslash \Omega|=0$, and

$$
Z_{\lambda, N}^{\beta}\left(f_{n_{k}}\right)(\cdot, s ; x) \longrightarrow Z_{\lambda, N}^{\beta}(f)(\cdot, s ; x), \quad \text { as } k \rightarrow \infty, \text { in } \gamma(H, \mathbb{B}),
$$

for every $x \in \Omega$ and $s \in \mathbb{Q} \cap[1 / N, N]$. Here $\Omega$ and $\left(n_{k}\right)_{k \in \mathbb{N}}$ do not depend on $N$.

Also, there exists an increasing sequence $\left(k_{j}\right)_{j \in \mathbb{N}} \subset \mathbb{N}$ and a subset $W$ of $\Omega$ with $|\Omega|=|W|$, such that

$$
Z_{\lambda, N}^{\beta}\left(f_{n_{k_{j}}}\right)(\cdot, s ; x) \longrightarrow\left[\widetilde{Z}_{\lambda, N}^{\beta}(f)(x)\right](s), \quad \text { as } j \rightarrow \infty, \text { in } \gamma(H, \mathbb{B}),
$$

for every $x \in W$ and $s \in \mathbb{Q} \cap[1 / N, N]$. Again, $W$ and $\left(k_{j}\right)_{j \in \mathbb{N}}$ do not depend on $N$.

We conclude that

$$
Z_{\lambda, N}^{\beta}(f)(\cdot, s, x)=\left[\widetilde{Z}_{\lambda, N}^{\beta}(f)(x)\right](s), \quad x \in W, s \in \mathbb{Q} \cap[1 / N, N] .
$$

This equality is understood in $\gamma(H, \mathbb{B})$. 
Hence, we can write

$$
\begin{aligned}
\mid\{x & \left.\in(0, \infty): P_{*}^{\lambda}\left(G_{\mathbb{B}}^{\lambda, \beta}(f)\right)(x)>\alpha\right\} \mid \\
& =\left|\bigcup_{M \in \mathbb{N}}\left\{x \in(0, \infty): \sup _{s \in[1 / M, M]}\left\|P_{s}^{\lambda}\left(G_{\mathbb{B}}^{\lambda, \beta}(f)\right)(x)\right\|_{\gamma(H, \mathbb{B})}>\alpha\right\}\right| \\
& \leq \lim _{M \rightarrow \infty}\left|\left\{x \in(0, \infty): \sup _{s \in[1 / M, M] \cap \mathbb{Q}}\left\|P_{s}^{\lambda}\left(G_{\mathbb{B}}^{\lambda, \beta}(f)\right)(x)\right\|_{\gamma(H, \mathbb{B})}>\alpha\right\}\right| \\
& \leq \lim _{M \rightarrow \infty}\left|\left\{x \in W: \sup _{s \in[1 / M, M] \cap \mathbb{Q}}\left\|\widetilde{Z}_{\lambda, M}^{\beta}(f)(\cdot, s, x)\right\|_{\gamma(H, \mathbb{B})}>\alpha\right\}\right| \\
& \leq \frac{C}{\alpha}\|f\|_{L^{1}((0, \infty), \mathbb{B}),} \quad \alpha>0 .
\end{aligned}
$$

Thus, we prove that the operator $Z_{\lambda}^{\beta}$ defined by

$$
Z_{\lambda}^{\beta}(f)(t, s ; x)=P_{s}^{\lambda}\left(G_{\mathbb{B}}^{\lambda, \beta}(f)(t, \cdot)\right)(x), \quad s, t, x \in(0, \infty),
$$

is bounded from $L^{1}((0, \infty), \mathbb{B})$ into $L^{1, \infty}\left((0, \infty), L^{\infty}((0, \infty), \gamma(H, \mathbb{B}))\right)$.

By proceeding in a similar way, since $L_{c}^{\infty}(0, \infty) \otimes \mathbb{B}$ is a dense subset of $H^{1}((0, \infty), \mathbb{B})$, we can see that $Z_{\lambda}^{\beta}$ defines a bounded operator from $H^{1}((0, \infty), \mathbb{B})$ into $L^{1}\left((0, \infty), L^{\infty}((0, \infty), \gamma(H, \mathbb{B}))\right)$. Here $L_{c}^{\infty}(0, \infty)$ represents the space of bounded measurable functions with compact support in $(0, \infty)$.

Thus, if $a$ is a 2-atom satisfying (Aii) we get that

$$
\left\|P_{s}^{\lambda}\left(G_{\mathbb{B}}^{\lambda, \beta}(a)\right)\right\|_{L^{1}\left((0, \infty), L^{\infty}((0, \infty), \gamma(H, \mathbb{B}))\right)} \leq C
$$

where $C$ does not depend on $a$.

On the other hand, by using (7) it follows that

$$
\left\|K_{\lambda}^{\beta}(\cdot, \cdot ; x, y)\right\|_{L^{\infty}((0, \infty), H)} \leq C \frac{(x y)^{\lambda}}{|x-y|^{2 \lambda+1}}, \quad x, y \in(0, \infty), x \neq y .
$$

The, since the operator $Z_{\lambda}^{\beta}$ is bounded from $L^{2}((0, \infty), \mathbb{B})$ into $L^{2}\left((0, \infty), L^{\infty}((0, \infty), \gamma(H, \mathbb{B}))\right)$, by proceeding as in the proof of 25 we can deduce that there exists $C>0$ such that, for every $\delta>0$, and $b \in \mathbb{B},\|b\|_{\mathbb{B}}=1$,

$$
\left\|P_{s}^{\lambda}\left(G_{\mathbb{B}}^{\lambda, \beta}(a)\right)\right\|_{L^{1}\left((0, \infty), L^{\infty}((0, \infty), \gamma(H, \mathbb{B}))\right)} \leq C
$$

when $a=b \chi_{(0, \delta)} / \delta$.

We conclude that, for every $f \in H_{o}^{1}((0, \infty), \mathbb{B})$,

$$
\left\|P_{*}^{\lambda}\left(G_{\mathbb{B}}^{\lambda, \beta}(f)\right)\right\|_{L^{1}(0, \infty)} \leq C\|f\|_{H_{\mathrm{o}}^{1}((0, \infty), \mathbb{B})} .
$$

2.2. We show now that $G_{\mathbb{B}}^{\lambda, \beta}$ is bounded from $B M O_{\mathrm{o}}((0, \infty), \mathbb{B})$ into $B M O_{\mathrm{o}}((0, \infty), \gamma(H, \mathbb{B}))$. This requires verifying the corresponding vector-valued conditions $(B i)$ and $(B i i)$ which we collected in Lemma 2.4 and Lemma 2.5, respectively.

Lemma 2.4. Consider $\mathbb{B}$ a UMD Banach space and $\beta, \lambda>0$. There exists $C>0$ such that, for every $r>0$,

$$
\frac{1}{r} \int_{0}^{r}\left\|G_{\mathbb{B}}^{\lambda, \beta}(f)(\cdot, x)\right\|_{\gamma(H, \mathbb{B})} d x \leq C\|f\|_{B M O_{\mathrm{o}}((0, \infty), \mathbb{B})}, \quad f \in B M O_{\mathrm{o}}((0, \infty), \mathbb{B}) .
$$


Proof. Assume that $f \in B M O_{\circ}((0, \infty), \mathbb{B})$. According to $[7)$, for every $k \in \mathbb{N}$, we have that

$$
\left|\partial_{t}^{k} P_{t}^{\lambda}(x, y)\right| \leq C \frac{(x y)^{\lambda}}{(t+|x-y|)^{2 \lambda+1+k}} \leq \frac{C}{t^{k}} \frac{(x y)^{\lambda}}{(t+|x-y|)^{2 \lambda+1}}, \quad t, x, y \in(0, \infty) .
$$

Then, for every

$$
\begin{aligned}
\int_{0}^{\infty} & \left|\partial_{t}^{k} P_{t}^{\lambda}(x, y)\right|\|f(y)\|_{\mathbb{B}} d y \leq \frac{C}{t^{k}} \int_{0}^{\infty} \frac{(x y)^{\lambda}}{(t+|x-y|)^{2 \lambda+1}}\|f(y)\|_{\mathbb{B}} d y \\
\leq & \frac{C}{t^{k}}\left\{\int_{0}^{2 x} \frac{x^{2 \lambda}}{t^{2 \lambda+1}}\|f(y)\|_{\mathbb{B}} d y+\int_{2 x}^{\infty} \frac{(x y)^{\lambda}}{y^{2 \lambda+1}}\|f(y)\|_{\mathbb{B}} d y\right\} \\
& \leq \frac{C}{t^{k}}\left\{\left(\frac{x}{t}\right)^{2 \lambda+1}\|f\|_{B M O_{o}((0, \infty), \mathbb{B})}+\sum_{j=1}^{\infty} x^{\lambda} \int_{2 x j^{2 / \lambda}}^{2 x(j+1)^{2 / \lambda}} \frac{1}{y^{\lambda+1}}\|f(y)\|_{\mathbb{B}} d y\right\} \\
& \leq \frac{C}{t^{k}}\left\{\left(\frac{x}{t}\right)^{2 \lambda+1}\|f\|_{B M O_{\circ}((0, \infty), \mathbb{B})}+\sum_{j=1}^{\infty} \frac{x^{\lambda}}{\left(x j^{2 / \lambda}\right)^{\lambda+1}} \int_{0}^{2 x(j+1)^{2 / \lambda}}\|f(y)\|_{\mathbb{B}} d y\right\} \\
& \leq \frac{C}{t^{k}}\left\{\left(\frac{x}{t}\right)^{2 \lambda+1}+\sum_{j=1}^{\infty} \frac{1}{j^{2}}\right\}\|f\|_{B M O_{o}((0, \infty), \mathbb{B})}, \quad t, x \in(0, \infty) \text { and } k \in \mathbb{N} .
\end{aligned}
$$

We can write

$$
\partial_{t}^{k} P_{t}^{\lambda}(f)(x)=\int_{0}^{\infty} \partial_{t}^{k} P_{t}^{\lambda}(x, y) f(y) d y, \quad t, x \in(0, \infty) \text { and } k \in \mathbb{N} .
$$

By using again (7), if $m \in \mathbb{N}$ such that $m-1 \leq \beta<m$, we get

$$
\begin{aligned}
& \int_{0}^{\infty} s^{m-\beta-1} \int_{0}^{\infty}\left|\partial_{t}^{m} P_{t+s}^{\lambda}(x, y)\right|\|f(y)\|_{\mathbb{B}} d y d s \\
& \quad \leq C\|f\|_{B M O_{\circ}(\mathbb{R}, \mathbb{B})} \int_{0}^{\infty} \frac{s^{m-\beta-1}}{(t+s)^{m}}\left\{\left(\frac{x}{t+s}\right)^{2 \lambda+1}+1\right\} d s<\infty, \quad t, x \in(0, \infty) .
\end{aligned}
$$

This leads to

$$
G_{\mathbb{B}}^{\lambda, \beta}(f)(t, x)=\int_{0}^{\infty} K_{\lambda}^{\beta}(t ; x, y) f(y) d y, \quad t, x \in(0, \infty),
$$

where $K_{\lambda}^{\beta}(t ; x, y)=t^{\beta} \partial_{t}^{\beta} P_{t}^{\lambda}(x, y), t, x, y \in(0, \infty)$.

Let $r>0$. We split $G_{\mathbb{B}}^{\lambda, \beta}(f)(t, x)$ as follows

$$
G_{\mathbb{B}}^{\lambda, \beta}(f)(t, x)=G_{\mathbb{B}, 1}^{\lambda, \beta}(f)(t, x)+G_{\mathbb{B}, 2}^{\lambda, \beta}(f)(t, x), \quad t, x \in(0, \infty),
$$

being

$$
G_{\mathbb{B}, 1}^{\lambda, \beta}(f)(t, x)=\int_{0}^{2 r} K_{\lambda}^{\beta}(t ; x, y) f(y) d y, \quad t, x \in(0, \infty) .
$$

Since $G_{\mathbb{B}}^{\lambda, \beta}$ is a bounded operator from $L^{2}((0, \infty), \mathbb{B})$ into $L^{2}((0, \infty), \gamma(H, \mathbb{B}))$ (Theorem $\mathbb{B}$ ), we obtain

$$
\begin{aligned}
\frac{1}{r} \int_{0}^{r}\left\|G_{\mathbb{B}, 1}^{\lambda, \beta}(f)(\cdot, x)\right\|_{\gamma(H, \mathbb{B})} d x & \leq\left(\frac{1}{r} \int_{0}^{\infty}\left\|G_{\mathbb{B}}^{\lambda, \beta}\left(f \chi_{(0,2 r)}\right)(\cdot, x)\right\|_{\gamma(H, \mathbb{B})}^{2} d x\right)^{1 / 2} \\
& \leq C\left(\frac{1}{r} \int_{0}^{2 r}\|f(y)\|_{\mathbb{B}}^{2} d y\right)^{1 / 2} \leq C\|f\|_{B M O_{o}((0, \infty), \mathbb{B}) .}
\end{aligned}
$$

Note that the last inequality follows from John-Niremberg's property. 
If $h \in H$, by using 23 and proceeding as in 31 we have

$$
\begin{aligned}
\int_{0}^{\infty} & \int_{2 r}^{\infty}\left|K_{\lambda}^{\beta}(t ; x, y)\right|\|f(y)\|_{\mathbb{B}} d y|h(t)| \frac{d t}{t} \leq\|h\|_{H} \int_{2 r}^{\infty}\left\|K_{\lambda}^{\beta}(\cdot ; x, y)\right\|_{H}\|f(y)\|_{\mathbb{B}} d y \\
& \leq C\|h\|_{H} \int_{2 r}^{\infty} \frac{(x y)^{\lambda}}{|x-y|^{2 \lambda+1}}\|f(y)\|_{\mathbb{B}} d y \leq C\|h\|_{H} x^{\lambda} \int_{2 r}^{\infty} \frac{1}{y^{\lambda+1}}\|f(y)\|_{\mathbb{B}} d y \\
& \leq C\|h\|_{H}\|f\|_{B M O_{\circ}((0, \infty), \mathbb{B})}, \quad x \in(0, r) .
\end{aligned}
$$

Then, if $\left(h_{j}\right)_{j=1}^{n}$ is a set of orthonormal functions in $H$, we can write

$$
\begin{aligned}
(\mathbb{E} & \left.\left\|\sum_{j=1}^{n} \gamma_{j} \int_{0}^{\infty} h_{j}(t) \int_{2 r}^{\infty} K_{\lambda}^{\beta}(t ; x, y) f(y) d y d t\right\|_{\mathbb{B}}^{2}\right)^{1 / 2} \\
& =\left(\mathbb{E}\left\|\int_{2 r}^{\infty} f(y) \sum_{j=1}^{n} \gamma_{j} \int_{0}^{\infty} K_{\lambda}^{\beta}(t ; x, y) h_{j}(t) d t d y\right\|_{\mathbb{B}}^{2}\right)^{1 / 2} \\
& \leq \int_{2 r}^{\infty}\|f(y)\|_{\mathbb{B}}\left(\mathbb{E}\left|\sum_{j=1}^{n} \gamma_{j} \int_{0}^{\infty} K_{\lambda}^{\beta}(t ; x, y) h_{j}(t) d t\right|^{2}\right)^{1 / 2} d y \\
& \leq \int_{2 r}^{\infty}\|f(y)\|_{\mathbb{B}}\left\|K_{\lambda}^{\beta}(\cdot ; x, y)\right\|_{\gamma(H, \mathbb{C})} d y .
\end{aligned}
$$

Since $\gamma(H, \mathbb{C})=H$ and again by 23 we get, for each $x \in(0, r)$,

$\left\|G_{\mathbb{B}, 2}^{\lambda, \beta}(f)\right\|_{\gamma(H, \mathbb{B})}=\left\|\int_{2 r}^{\infty} K_{\lambda}^{\beta}(\cdot ; x, y) f(y) d y\right\|_{\gamma(H, \mathbb{B})} \leq C \int_{2 r}^{\infty}\|f(y)\|_{\mathbb{B}}\left\|K_{\lambda}^{\beta}(\cdot ; x, y)\right\|_{H} d y \leq C\|f\|_{B M O_{\circ}((0, \infty), \mathbb{B})}$.

Hence,

$$
\frac{1}{r} \int_{0}^{r}\left\|G_{\mathbb{B}, 2}^{\lambda, \beta}(f)(\cdot, x)\right\|_{\gamma(H, \mathbb{B})} d x \leq C\|f\|_{B M O_{\circ}((0, \infty), \mathbb{B})} .
$$

From 32 and 33 we conclude the proof of this Lemma.

Note that 30 implies that $\left\|G_{\mathbb{B}}^{\lambda, \beta}(f)(\cdot, x)\right\|_{\gamma(H, \mathbb{B})}<\infty$, a.e. $x \in(0, \infty)$.

Lemma 2.5. Let $\mathbb{B}$ be a UMD Banach space and $\beta, \lambda>0$. The operator $G_{\mathbb{B}}^{\lambda, \beta}$ is bounded from $B M O_{\circ}((0, \infty), \mathbb{B})$ into $B M O((0, \infty), \gamma(H, \mathbb{B}))$.

Proof. Let $f \in B M O_{\mathrm{o}}((0, \infty), \mathbb{B})$. We consider the odd extension function $f_{\mathrm{o}}$ of $f$ to $\mathbb{R}$ and

$$
G_{\mathbb{B}}^{\beta}\left(f_{\mathrm{o}}\right)(t, x)=\int_{\mathbb{R}} K^{\beta}(t ; x, y) f_{\mathrm{o}}(y) d y, \quad t \in(0, \infty) \text { and } x \in \mathbb{R},
$$

where

$$
K^{\beta}(t ; x, y)=t^{\beta} \partial_{t}^{\beta} P_{t}(x-y), \quad t \in(0, \infty) \text { and } x, y \in \mathbb{R} .
$$

Here $P_{t}(z)=t /\left[\pi\left(t^{2}+z^{2}\right)\right], t \in(0, \infty)$ and $z \in \mathbb{R}$, is the classical Poisson semigroup. 
We can write

$$
\begin{aligned}
G_{\mathbb{B}}^{\beta}\left(f_{\mathrm{o}}\right)(t, x)= & \int_{0}^{\infty} t^{\beta} \partial_{t}^{\beta} P_{t}(x-y) f(y) d y-\int_{0}^{\infty} t^{\beta} \partial_{t}^{\beta} P_{t}(x+y) f(y) d y \\
= & \int_{x / 2}^{2 x} t^{\beta} \partial_{t}^{\beta} P_{t}(x-y) f(y) d y-\int_{x / 2}^{2 x} t^{\beta} \partial_{t}^{\beta} P_{t}(x+y) f(y) d y \\
& +\int_{0}^{x / 2} t^{\beta} \partial_{t}^{\beta}\left[P_{t}(x-y)-P_{t}(x+y)\right] f(y) d y+\int_{2 x}^{\infty} t^{\beta} \partial_{t}^{\beta}\left[P_{t}(x-y)-P_{t}(x+y)\right] f(y) d y \\
= & \sum_{j=1}^{4} I_{j}(f)(t, x), \quad t, x \in(0, \infty) .
\end{aligned}
$$

In [5, Lemma 1] it was established that, if $m \in \mathbb{N}$ is such that $m-1 \leq \beta<m$,

$$
t^{\beta} \partial_{t}^{\beta} P_{t}(z)=\sum_{k \in \mathbb{N}, 0 \leq k \leq(m+1) / 2} \frac{c_{k}}{t} \varphi^{k}\left(\frac{z}{t}\right), \quad t \in(0, \infty) \text { and } z \in \mathbb{R},
$$

where, for every $k \in \mathbb{N}, 0 \leq k \leq(m+1) / 2, c_{k} \in \mathbb{C}$ and

$$
\varphi^{k}(z)=\int_{0}^{\infty} \frac{(1+v)^{m+1-2 k} v^{m-\beta-1}}{\left[(1+v)^{2}+z^{2}\right]^{m-k+1}} d v, \quad z \in \mathbb{R} .
$$

Let $k \in \mathbb{N}$ such that $0 \leq k \leq(m+1) / 2$. We have that

$$
\begin{aligned}
& \frac{1}{t} \varphi^{k}\left(\frac{x+y}{t}\right)-\frac{1}{t} \varphi^{k}\left(\frac{x-y}{t}\right)=t^{2(m-k)+1} \int_{0}^{\infty}(1+v)^{m+1-2 k} v^{m-\beta-1} \\
& \times\left[\frac{1}{\left[(1+v)^{2} t^{2}+(x+y)^{2}\right]^{m-k+1}}-\frac{1}{\left[(1+v)^{2} t^{2}+(x-y)^{2}\right]^{m-k+1}}\right] d v \\
& =t^{2(m-k)+1} \sum_{\ell=0}^{m-k+1}\left(\begin{array}{c}
m-k+1 \\
\ell
\end{array}\right) \int_{0}^{\infty}(1+v)^{m+1-2 k} v^{m-\beta-1} \\
& \quad \times \frac{[(1+v) t]^{2(m-k+1-\ell)}\left[(x-y)^{2 \ell}-(x+y)^{2 \ell}\right]}{\left[\left((1+v)^{2} t^{2}+(x+y)^{2}\right)\left((1+v)^{2} t^{2}+(x-y)^{2}\right)\right]^{m-k+1}} d v, \quad t, x, y \in(0, \infty) .
\end{aligned}
$$

We get

$$
\begin{aligned}
& \left|\frac{1}{t} \varphi^{k}\left(\frac{x+y}{t}\right)-\frac{1}{t} \varphi^{k}\left(\frac{x-y}{t}\right)\right| \leq C y t^{2(m-k)+1} \sum_{\ell=1}^{m-k+1} x^{2 \ell-1} \int_{0}^{\infty}(1+v)^{m+1-2 k} v^{m-\beta-1} \\
& \quad \times \frac{[(1+v) t]^{2(m-k+1-\ell)}}{[(1+v) t+x]^{4(m-k+1)}} d v \\
& \leq C y t^{2(m-k)+1} \int_{0}^{\infty} \frac{(1+v)^{m+1-2 k} v^{m-\beta-1}}{[(1+v) t+x]^{2(m-k)+3}} d v, \quad t \in(0, \infty), 0<y<x / 2 .
\end{aligned}
$$

Minkowski's inequality leads to

$$
\begin{aligned}
\left\|\frac{1}{t} \varphi^{k}\left(\frac{x+y}{t}\right)-\frac{1}{t} \varphi^{k}\left(\frac{x-y}{t}\right)\right\|_{H} & \leq C y \int_{0}^{\infty}(1+v)^{m+1-2 k} v^{m-\beta-1} \\
& \times\left(\int_{0}^{\infty} \frac{t^{4(m-k)+1}}{[(1+v) t+x]^{4(m-k)+6}} d t\right)^{1 / 2} d v \\
& \leq C \frac{y}{x^{2}} \int_{0}^{\infty} \frac{v^{m-\beta-1}}{(1+v)^{m}} d v \\
& \leq C \frac{y}{x^{2}}, \quad 0<y<x / 2 .
\end{aligned}
$$

Hence, we obtain

$$
\left\|t^{\beta} \partial_{t}^{\beta}\left[P_{t}(x-y)-P_{t}(x+y)\right]\right\|_{H} \leq C \frac{y}{x^{2}}, \quad 0<y<x / 2
$$


It follows that

$$
\left\|I_{3}(f)(\cdot, x)\right\|_{\gamma(H, \mathbb{B}))} \leq \frac{C}{x^{2}} \int_{0}^{x / 2} y\|f(y)\|_{\mathbb{B}} d y \leq C\|f\|_{B M O_{\circ}((0, \infty), \mathbb{B})}, \quad x \in(0, \infty) .
$$

By symmetries, we also get

$$
\left\|t^{\beta} \partial_{t}^{\beta}\left[P_{t}(x-y)-P_{t}(x+y)\right]\right\|_{H} \leq C \frac{x}{y^{2}}, \quad 0<2 x<y
$$

and then

$$
\begin{aligned}
\left\|I_{4}(f)(\cdot, x)\right\|_{\gamma(H, \mathbb{B}))} & \leq C x \int_{2 x}^{\infty} \frac{\|f(y)\|_{\mathbb{B}}}{y^{2}} d y \leq C x \sum_{k=1}^{\infty} \int_{2 k^{2} x}^{2(k+1)^{2} x} \frac{\|f(y)\|_{\mathbb{B}}}{y^{2}} d y \\
& \leq C \sum_{k=1}^{\infty} \frac{1}{x k^{4}} \int_{0}^{2(k+1)^{2} x}\|f(y)\|_{\mathbb{B}} d y \leq C\|f\|_{B M O_{\circ}((0, \infty), \mathbb{B}), \quad x \in(0, \infty) .}
\end{aligned}
$$

Note that to establish

$$
\left\|I_{3}(f)(\cdot, x)\right\|_{\gamma(H, \mathbb{B}))} \leq C\|f\|_{B M O_{\circ}((0, \infty), \mathbb{B})}, \quad x \in(0, \infty)
$$

it is enough to use that

$$
\left\|t^{\beta} \partial_{t}^{\beta}\left[P_{t}(x-y)-P_{t}(x+y)\right]\right\|_{H} \leq\left\|t^{\beta} \partial_{t}^{\beta} P_{t}(x-y)\right\|_{H}+\left\|t^{\beta} \partial_{t}^{\beta} P_{t}(x+y)\right\|_{H} \leq \frac{C}{x}, \quad 0<y<\frac{x}{2} .
$$

However, the estimation

$$
\left\|t^{\beta} \partial_{t}^{\beta}\left[P_{t}(x-y)-P_{t}(x+y)\right]\right\|_{H} \leq \frac{C}{y}, \quad 0<2 x<y,
$$

does not allow to show that

$$
\left\|I_{4}(f)(\cdot, x)\right\|_{\gamma(H, \mathbb{B}))} \leq C\|f\|_{B M O_{\circ}((0, \infty), \mathbb{B})}, \quad x \in(0, \infty)
$$

Also, we obtain

$$
\begin{aligned}
\left\|t^{\beta} \partial_{t}^{\beta} P_{t}(x+y)\right\|_{H} & \leq C \sum_{k \in \mathbb{N}, 0 \leq k \leq(m+1) / 2} \int_{0}^{\infty}(1+v)^{m+1-2 k} v^{m-\beta-1}\left(\int_{0}^{\infty} \frac{t^{4(m-k)+1}}{[(1+v) t+x+y]^{4(m-k)+4}} d t\right)^{1 / 2} d v \\
& \leq \frac{C}{x+y}, \quad x, y \in(0, \infty)
\end{aligned}
$$

Hence,

$$
\left\|I_{2}(f)(\cdot, x)\right\|_{\gamma(H, \mathbb{B}))} \leq \frac{C}{x} \int_{x / 2}^{2 x}\|f(y)\|_{\mathbb{B}} d y \leq C\|f\|_{B M O_{\circ}((0, \infty), \mathbb{B})}, \quad x \in(0, \infty) .
$$

We conclude that

$$
G_{\mathbb{B}}^{\beta}\left(f_{\mathrm{o}}\right)(t, x)-\int_{x / 2}^{2 x} t^{\beta} \partial_{t}^{\beta} P_{t}(x-y) f(y) d y \in L^{\infty}((0, \infty), \gamma(H, \mathbb{B})),
$$

and we deduce that $G_{\mathbb{B}}^{\beta}\left(f_{\mathrm{o}}\right) \in B M O((0, \infty), \gamma(H, \mathbb{B}))$ if, and only if, $G_{\mathbb{B}, \text { loc }}^{\beta}(f) \in B M O((0, \infty), \gamma(H, \mathbb{B}))$, where

$$
G_{\mathbb{B}, \text { loc }}^{\beta}(f)(t, x)=\int_{x / 2}^{2 x} t^{\beta} \partial_{t}^{\beta} P_{t}(x-y) f(y) d y, \quad t, x \in(0, \infty) .
$$

We are going to prove that $G_{\mathbb{B}}^{\beta}\left(f_{\mathrm{o}}\right) \in B M O((0, \infty), \gamma(H, \mathbb{B}))$. Let $0<r<s<\infty$. We define $I=(r, s), x_{I}=(r+s) / 2$ and $d_{I}=(s-r) / 2$. We decompose $f_{\mathrm{o}}$ as follows:

$$
f_{\mathrm{o}}=\left(f_{\mathrm{o}}-f_{I}\right) \chi_{2 I}+\left(f_{\mathrm{o}}-f_{I}\right) \chi_{(0, \infty) \backslash 2 I}+f_{I}=f_{1}+f_{2}+f_{3},
$$


where $2 I=\left(x_{I}-2 d_{I}, x_{I}+2 d_{I}\right)$. By [28, Theorem 4.2] and [6], proof of Theorem 1.2], it follows that the operator $G_{\mathbb{B}}^{\beta}$ is bounded from $L^{2}(\mathbb{R}, \mathbb{B})$ into $L^{2}(\mathbb{R}, \gamma(H, \mathbb{B}))$. Then,

$$
\begin{gathered}
\frac{1}{|I|} \int_{I}\left\|G_{\mathbb{B}}^{\beta}\left(f_{1}\right)(\cdot, x)\right\|_{\gamma(H, \mathbb{B})} d x \leq\left(\frac{1}{|I|} \int_{I}\left\|G_{\mathbb{B}}^{\beta}\left(f_{1}\right)(\cdot, x)\right\|_{\gamma(H, \mathbb{B})}^{2} d x\right)^{1 / 2} \\
\leq C\left(\frac{1}{|I|} \int_{2 I}\left\|f_{\mathrm{o}}(x)-f_{I}\right\|_{\mathbb{B}}^{2} d x\right)^{1 / 2} \leq C\|f\|_{B M O_{\mathrm{o}}((0, \infty), \mathbb{B}) .}
\end{gathered}
$$

Hence,

$$
\left\|G_{\mathbb{B}}^{\beta}\left(f_{1}\right)(\cdot, x)\right\|_{\gamma(H, \mathbb{B})}<\infty, \quad \text { a.e. } x \in(0, \infty) .
$$

On the other hand, since

$$
\int_{\mathbb{R}} P_{t}(x-y) d y=1, \quad t \in(0, \infty) \text { and } x \in \mathbb{R},
$$

if $m \in \mathbb{N}$, being $m-1 \leq \beta<m$,

$\partial_{t}^{\beta} \int_{\mathbb{R}} P_{t}(x-y) d y=\frac{e^{-i \pi(m-\beta)}}{\Gamma(m-\beta)} \int_{0}^{\infty} s^{m-\beta-1} \partial_{t}^{m} \int_{\mathbb{R}} P_{t+s}(x-y) d y d s=0, \quad t \in(0, \infty)$ and $x \in \mathbb{R}$.

It follows that $G_{\mathbb{B}}^{\beta}\left(f_{3}\right)=0$.

In [6, Section 3.1] it was proved that

$$
\left\|K_{\lambda}^{\beta}(\cdot ; x, y)-K^{\beta}(\cdot ; x, y)\right\|_{H} \leq \frac{C}{x}, \quad x / 2<y<2 x, x \in(0, \infty) .
$$

Then,

$$
\left\|\int_{x / 2}^{2 x}\left(K_{\lambda}^{\beta}(\cdot ; x, y)-K^{\beta}(\cdot ; x, y)\right) f(y) d y\right\|_{\gamma(H, \mathbb{B})} \leq \int_{x / 2}^{2 x}\left\|K_{\lambda}^{\beta}(\cdot ; x, y)-K^{\beta}(\cdot ; x, y)\right\|_{H}\|f(y)\|_{\mathbb{B}} d y
$$

$$
\leq \frac{C}{x} \int_{x / 2}^{2 x}\|f(y)\|_{\mathbb{B}} d y \leq C\|f\|_{B M O_{\circ}((0, \infty), \mathbb{B})}, \quad x \in(0, \infty) .
$$

Moreover, by using 23] and as it was seen in 31,

$$
\left\|\int_{2 x}^{\infty} K_{\lambda}^{\beta}(\cdot ; x, y) f(y) d y\right\|_{\gamma(H, \mathbb{B})} \leq C x^{\lambda} \int_{2 x}^{\infty}\|f(y)\|_{\mathbb{B}} \frac{d y}{y^{\lambda+1}} \leq C\|f\|_{B M O_{o}((0, \infty), \mathbb{B})}, \quad x \in(0, \infty),
$$

and

(40)

$$
\left\|\int_{0}^{x / 2} K_{\lambda}^{\beta}(\cdot ; x, y) f(y) d y\right\|_{\gamma(H, \mathbb{B})} \leq \frac{C}{x} \int_{0}^{x / 2}\|f(y)\|_{\mathbb{B}} d y \leq C\|f\|_{B M O_{\circ}((0, \infty), \mathbb{B})}, \quad x \in(0, \infty) .
$$

Hence,

$$
\left\|G_{\mathbb{B}}^{\lambda, \beta}(f)(\cdot, x)-G_{\mathbb{B}}^{\beta}\left(f_{\mathrm{o}}\right)(\cdot, x)\right\|_{\gamma(H, \mathbb{B})} \leq C\|f\|_{B M O_{\mathrm{o}}((0, \infty), \mathbb{B})}, \quad x \in(0, \infty) .
$$

Since, $\left\|G_{\mathbb{B}}^{\lambda, \beta}(f)(\cdot, x)\right\|_{\gamma(H, \mathbb{B})}<\infty$ and $\left\|G_{\mathbb{B}}^{\beta}\left(f_{1}\right)(\cdot, x)\right\|_{\gamma(H, \mathbb{B})}<\infty$, a.e. $x \in(0, \infty)$, also

$$
\left\|G_{\mathbb{B}}^{\beta}\left(f_{2}\right)(\cdot, x)\right\|_{\gamma(H, \mathbb{B})}<\infty, \quad \text { a.e. } x \in(0, \infty) .
$$

By proceeding as in the proof of 15 we get

$$
\left\|\partial_{x} K^{\beta}(\cdot ; x, y)\right\|_{H} \leq \frac{C}{|x-y|^{2}}, \quad x, y \in(0, \infty), x \neq y .
$$


We choose $x_{0} \in I$ such that $\left\|G_{\mathbb{B}}^{\beta}\left(f_{2}\right)\left(\cdot, x_{0}\right)\right\|_{\gamma(H, \mathbb{B})}<\infty$. By using 441] we obtain

$$
\begin{aligned}
& \frac{1}{|I|} \int_{I}\left\|G_{\mathbb{B}}^{\beta}\left(f_{2}\right)(\cdot, x)-G_{\mathbb{B}}^{\beta}\left(f_{2}\right)\left(\cdot, x_{0}\right)\right\|_{\gamma(H, \mathbb{B})} d x \\
& \quad \leq \frac{C}{|I|} \int_{I} \int_{(0, \infty) \backslash 2 I}\left\|f_{\mathrm{o}}(y)-f_{I}\right\|_{\mathbb{B}}\left\|K^{\beta}(\cdot ; x, y)-K^{\beta}\left(\cdot ; x_{0}, y\right)\right\|_{H} d y d x \\
& \quad \leq \frac{C}{|I|} \int_{I} \int_{(0, \infty) \backslash 2 I}\left\|f_{\mathrm{o}}(y)-f_{I}\right\|_{\mathbb{B}}\left|\int_{x}^{x_{0}}\left\|\partial_{u} K^{\beta}(\cdot, u, y)\right\|_{H} d u\right| d y d x \\
& \quad \leq \frac{C}{|I|} \int_{I} \int_{(0, \infty) \backslash 2 I}\left\|f_{\mathrm{o}}(y)-f_{I}\right\|_{\mathbb{B}} \frac{\left|x-x_{0}\right|}{|x-y|^{2}} d y d x
\end{aligned}
$$

By employing standard arguments (see, for instance, [12, (9)]) we deduce that

$$
\frac{1}{|I|} \int_{I}\left\|G_{\mathbb{B}}^{\beta}\left(f_{2}\right)(\cdot, x)-G_{\mathbb{B}}^{\beta}\left(f_{2}\right)\left(\cdot, x_{0}\right)\right\|_{\gamma(H, \mathbb{B})} d x \leq C\|f\|_{B M O_{o}((0, \infty), \mathbb{B})} .
$$

By putting together (36), 37) and 42 we conclude that

$$
\frac{1}{|I|} \int_{I}\left\|G_{\mathbb{B}}^{\beta}\left(f_{\mathrm{o}}\right)(\cdot, x)-G_{\mathbb{B}}^{\beta}\left(f_{2}\right)\left(\cdot, x_{0}\right)\right\|_{\gamma(H, \mathbb{B})} d x \leq C\|f\|_{B M O_{o}((0, \infty), \mathbb{B})},
$$

where $C>0$ does not depend on $I$. Hence, $G_{\mathbb{B}}^{\beta}\left(f_{\mathrm{o}}\right) \in B M O((0, \infty), \gamma(H, \mathbb{B}))$, and then $G_{\mathbb{B}, \text { loc }}^{\beta}(f) \in B M O((0, \infty), \gamma(H, \mathbb{B}))$. Finally by using $[38$, , 39] and 40 we obtain that

$$
\left\|G_{\mathbb{B}}^{\lambda, \beta}(f)(\cdot, x)-G_{\mathbb{B}, \mathrm{loc}}^{\beta}(f)(\cdot, x)\right\|_{\gamma(H, \mathbb{B})} \leq C\|f\|_{B M O_{\circ}((0, \infty), \mathbb{B})}, \quad x \in(0, \infty),
$$

and hence $G_{\mathbb{B}}^{\lambda, \beta}(f) \in B M O((0, \infty), \gamma(H, \mathbb{B}))$.

2.3. In this section we are going to show that

$$
\|f\|_{B M O_{\circ}((0, \infty), \mathbb{B})} \leq C\left\|G_{\mathbb{B}}^{\lambda, \beta}(f)\right\|_{B M O_{\circ}((0, \infty), \gamma(H, \mathbb{B}))}, \quad f \in B M O_{\circ}((0, \infty), \mathbb{B}) .
$$

To establish this property we need to prove some auxiliary results.

Suppose firstly that $f \in B M O_{\mathrm{o}}(0, \infty)$. According to [7, Theorem 6.1] we have that the measure

$$
d \mu_{f}(x, t)=\left|t^{\beta} \partial_{t}^{\beta} P_{t}^{\lambda}(f)(x)\right|^{2} \frac{d x d t}{t}
$$

is Carleson on $(0, \infty)^{2}$. Then, by [7, Proposition 5.3], for every $a \in L_{+c}^{\infty}(0, \infty)$, where $L_{+c}^{\infty}(0, \infty)$ denotes the space of bounded measurable functions with upper bounded support on $(0, \infty)$,

$$
\int_{0}^{\infty} \int_{0}^{\infty} t^{\beta} \partial_{t}^{\beta} P_{t}^{\lambda}(f)(x) t^{\beta} \partial_{t}^{\beta} P_{t}^{\lambda}(a)(x) \frac{d x d t}{t}=\frac{e^{2 \pi i \beta} \Gamma(2 \beta)}{2^{2 \beta}} \int_{0}^{\infty} f(x) a(x) d x .
$$

The following is a vector-valued version of 43 .

Proposition 2.2. Let $\lambda, \beta>0$. If $f \in B M O_{\circ}((0, \infty), \mathbb{B})$ and $a \in L_{+c}^{\infty}(0, \infty) \otimes \mathbb{B}^{*}$, then

$$
\int_{0}^{\infty} \int_{0}^{\infty}\left\langle G_{\mathbb{B}^{*}}^{\lambda, \beta}(a)(t, x), G_{\mathbb{B}}^{\lambda, \beta}(f)(t, x)\right\rangle_{\mathbb{B}^{*}, \mathbb{B}} \frac{d x d t}{t}=\frac{e^{2 \pi i \beta} \Gamma(2 \beta)}{2^{2 \beta}} \int_{0}^{\infty}\langle a(x), f(x)\rangle_{\mathbb{B}^{*}, \mathbb{B}} d x .
$$


Proof. Assume that $f \in B M O_{\mathrm{o}}((0, \infty), \mathbb{B})$ and $a \in L_{+c}^{\infty}(0, \infty) \otimes \mathbb{B}^{*}$. If $a=\sum_{j=1}^{n} a_{j} b_{j}$, being $a_{j} \in L_{+c}^{\infty}(0, \infty)$ and $b_{j} \in \mathbb{B}^{*}, j=1, \ldots, n$, by using 43 we can write

$$
\begin{aligned}
\int_{0}^{\infty} & \int_{0}^{\infty}\left\langle G_{\mathbb{B}^{*}}^{\lambda, \beta}(a)(t, x), G_{\mathbb{B}}^{\lambda, \beta}(f)(t, x)\right\rangle_{\mathbb{B}^{*}, \mathbb{B}} \frac{d x d t}{t} \\
& =\sum_{j=1}^{n} \int_{0}^{\infty} \int_{0}^{\infty} G_{\mathbb{C}}^{\lambda, \beta}\left(a_{j}\right)(t, x)\left\langle b_{j}, G_{\mathbb{B}}^{\lambda, \beta}(f)(t, x)\right\rangle_{\mathbb{B}^{*}, \mathbb{B}} \frac{d x d t}{t} \\
& =\sum_{j=1}^{n} \int_{0}^{\infty} \int_{0}^{\infty} G_{\mathbb{C}}^{\lambda, \beta}\left(a_{j}\right)(t, x) G_{\mathbb{C}}^{\lambda, \beta}\left(\left\langle b_{j}, f\right\rangle_{\mathbb{B}^{*}, \mathbb{B}}\right)(t, x) \frac{d x d t}{t} \\
& =\frac{e^{2 \pi i \beta} \Gamma(2 \beta)}{2^{2 \beta}} \sum_{j=1}^{n} \int_{0}^{\infty} a_{j}(x)\left\langle b_{j}, f(x)\right\rangle_{\mathbb{B}^{*}, \mathbb{B}} d x=\frac{e^{2 \pi i \beta} \Gamma(2 \beta)}{2^{2 \beta}} \int_{0}^{\infty}\langle a(x), f(x)\rangle_{\mathbb{B}^{*}, \mathbb{B}} d x,
\end{aligned}
$$

because, for every $j=1, \ldots, n,\left\langle b_{j}, f\right\rangle_{\mathbb{B}^{*}, \mathbb{B}} \in B M O_{\circ}(0, \infty)$.

Let $f \in B M O_{\mathrm{o}}((0, \infty), \mathbb{B})$. We have that

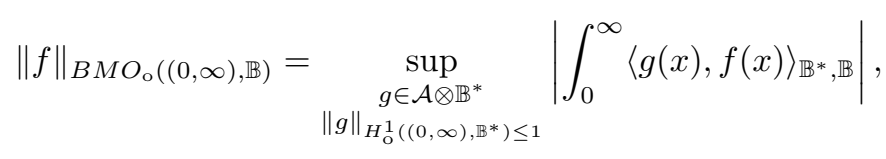

where $\mathcal{A}=\operatorname{span}\{a: a$ is an $\infty$-atom $\}$. Note that, since $\mathbb{B}$ is a UMD space, $\mathbb{B}^{*}$ is also UMD, $\mathbb{B}$ is reflexive and $B M O_{\circ}((0, \infty), \mathbb{B})$ is the dual space of $H_{\mathrm{o}}^{1}\left((0, \infty), \mathbb{B}^{*}\right)$. Moreover, since $\mathcal{A}$ is a dense subspace of $H_{\mathrm{o}}^{1}((0, \infty)), \mathcal{A} \otimes \mathbb{B}^{*}$ is dense in $H_{\mathrm{o}}^{1}\left((0, \infty), \mathbb{B}^{*}\right)$.

According to Proposition 2.2 we get, for every $a \in \mathcal{A} \otimes \mathbb{B}^{*}$,

$$
\int_{0}^{\infty} \int_{0}^{\infty}\left\langle G_{\mathbb{B}^{*}}^{\lambda, \beta}(a)(t, x), G_{\mathbb{B}}^{\lambda, \beta}(f)(t, x)\right\rangle_{\mathbb{B}^{*}, \mathbb{B}} \frac{d x d t}{t}=\frac{e^{2 \pi i \beta} \Gamma(2 \beta)}{2^{2 \beta}} \int_{0}^{\infty}\langle a(x), f(x)\rangle_{\mathbb{B}^{*}, \mathbb{B}} d x .
$$

Also, for every $a \in \mathcal{A} \otimes \mathbb{B}^{*}$, we can write

$$
\int_{0}^{\infty}\left\langle G_{\mathbb{B}^{*}}^{\lambda, \beta}(a)(t, x), G_{\mathbb{B}}^{\lambda, \beta}(f)(t, x)\right\rangle_{\mathbb{B}^{*}, \mathbb{B}} \frac{d t}{t}=\left\langle G_{\mathbb{B}^{*}}^{\lambda, \beta}(a)(\cdot, x), G_{\mathbb{B}}^{\lambda, \beta}(f)(\cdot, x)\right\rangle_{\gamma\left(H, \mathbb{B}^{*}\right), \gamma(H, \mathbb{B})}, \quad x \in(0, \infty) .
$$

Indeed, we take $a=\sum_{j=1}^{n} a_{j} b_{j}$, where $a_{j} \in \mathcal{A}$ and $b_{j} \in \mathbb{B}^{*}, j=1, \ldots, n$. By taking into account the results in Section 2.1, we have that

$$
G_{\mathbb{B}^{*}}^{\lambda, \beta}(a)(t, x)=\sum_{j=1}^{n} b_{j} G_{\mathbb{C}}^{\lambda, \beta}\left(a_{j}\right)(t, x) \in H_{\mathrm{o}}^{1}\left((0, \infty), \gamma\left(H, \mathbb{B}^{*}\right)\right) .
$$


The dual $(\gamma(H, \mathbb{B}))^{*}$ of $\gamma(H, \mathbb{B})$ can be identified with $\gamma\left(H, \mathbb{B}^{*}\right)$ by using the trace functional. If $\left(h_{m}\right)_{m \in \mathbb{N}}$ is an orthonormal basis in $H$ we can write

$$
\begin{aligned}
\left\langle G_{\mathbb{B}^{*}}^{\lambda, \beta}\right. & \left.(a)(\cdot, x), G_{\mathbb{B}}^{\lambda, \beta}(f)(\cdot, x)\right\rangle_{\gamma\left(H, \mathbb{B}^{*}\right), \gamma(H, \mathbb{B})}=\sum_{j=1}^{n}\left\langle b_{j} G_{\mathbb{C}}^{\lambda, \beta}\left(a_{j}\right)(\cdot, x), G_{\mathbb{B}}^{\lambda, \beta}(f)(\cdot, x)\right\rangle_{\gamma\left(H, \mathbb{B}^{*}\right), \gamma(H, \mathbb{B})} \\
= & \sum_{j=1}^{n} \sum_{m \in \mathbb{N}} \int_{0}^{\infty} h_{m}(t) \int_{0}^{\infty}\left\langle b_{j} G_{\mathbb{C}}^{\lambda, \beta}\left(a_{j}\right)(u, x), G_{\mathbb{B}}^{\lambda, \beta}(f)(t, x)\right\rangle_{\mathbb{B}^{*}, \mathbb{B}} h_{m}(u) \frac{d u}{u} \frac{d t}{t} \\
= & \sum_{j=1}^{n} \sum_{m \in \mathbb{N}} \int_{0}^{\infty} h_{m}(t) \int_{0}^{\infty} G_{\mathbb{C}}^{\lambda, \beta}\left(a_{j}\right)(u, x) G_{\mathbb{C}}^{\lambda, \beta}\left(\left\langle b_{j}, f\right\rangle_{\mathbb{B}^{*}, \mathbb{B}}\right)(t, x) h_{m}(u) \frac{d u}{u} \frac{d t}{t} \\
= & \sum_{j=1}^{n}\left\langle G_{\mathbb{C}}^{\lambda, \beta}\left(a_{j}\right)(\cdot, x), G_{\mathbb{C}}^{\lambda, \beta}\left(\left\langle b_{j}, f\right\rangle_{\mathbb{B}^{*}, \mathbb{B}}\right)(\cdot, x)\right\rangle_{\gamma(H, \mathbb{C}), \gamma(H, \mathbb{C})} \\
= & \sum_{j=1}^{n} \int_{0}^{\infty} G_{\mathbb{C}}^{\lambda, \beta}\left(a_{j}\right)(u, x) G_{\mathbb{C}}^{\lambda, \beta}\left(\left\langle b_{j}, f\right\rangle_{\mathbb{B}^{*}, \mathbb{B}}\right)(u, x) \frac{d u}{u} \\
= & \int_{0}^{\infty}\left\langle G_{\mathbb{B}^{*}}^{\lambda, \beta}(a)(u, x), G_{\mathbb{B}}^{\lambda, \beta}(f)(u, x)\right\rangle_{\mathbb{B}^{*}, \mathbb{B}} \frac{d u}{u}, \quad x \in(0, \infty) .
\end{aligned}
$$

We have used that $\gamma(H, \mathbb{C})=H$.

By 45 we obtain

$$
\begin{aligned}
\int_{0}^{\infty} & \left|\left\langle G_{\mathbb{B}^{*}}^{\lambda, \beta}(a)(\cdot, x), G_{\mathbb{B}}^{\lambda, \beta}(f)(\cdot, x)\right\rangle_{\gamma\left(H, \mathbb{B}^{*}\right), \gamma(H, \mathbb{B})}\right| d x \\
& \leq \sum_{j=1}^{n} \int_{0}^{\infty} \int_{0}^{\infty}\left|G_{\mathbb{C}}^{\lambda, \beta}\left(a_{j}\right)(t, x)\right|\left|G_{\mathbb{C}}^{\lambda, \beta}\left(\left\langle b_{j}, f\right\rangle_{\mathbb{B}^{*}, \mathbb{B}}\right)(t, x)\right| \frac{d t}{t} d x .
\end{aligned}
$$

Since, $\left\langle b_{j}, f\right\rangle_{\mathbb{B}^{*}, \mathbb{B}} \in B M O_{\circ}(0, \infty)$, for every $j=1, \ldots, n$, by [7, Theorem 6.1], $\left|G_{\mathbb{C}}^{\lambda, \beta}\left(\left\langle b_{j}, f\right\rangle_{\mathbb{B}^{*}, \mathbb{B}}\right)(t, x)\right|^{2} d t d x / t$ is a Carleson measure on $(0, \infty)^{2}$, for every $j=1, \ldots, n$. Then, according to [7, Propositions 5.1 and 5.2],

$$
\int_{0}^{\infty}\left|\left\langle G_{\mathbb{B}^{*}}^{\lambda, \beta}(a)(\cdot, x), G_{\mathbb{B}}^{\lambda, \beta}(f)(\cdot, x)\right\rangle_{\gamma\left(H, \mathbb{B}^{*}\right), \gamma(H, \mathbb{B})}\right| d x<\infty .
$$

From [4, Proposition 2.5] (adapted to this Bessel context), Proposition 2.2, 45], and by taking into account, as it has already been proved, that $G_{\mathbb{B}^{*}}^{\lambda, \beta}(a) \in H_{\mathrm{o}}^{1}\left((0, \infty), \gamma\left(H, \mathbb{B}^{*}\right)\right)$ (Section 2.1 ) and $G_{\mathbb{B}}^{\lambda, \beta}(f) \in B M O_{\mathrm{o}}((0, \infty), \gamma(H, \mathbb{B}))$ (Section 2.2), we conclude that

$$
\begin{aligned}
\left|\int_{0}^{\infty}\langle a(x), f(x)\rangle_{\mathbb{B}^{*}, \mathbb{B}} d x\right| & \leq C\left\|G_{\mathbb{B}^{*}}^{\lambda, \beta}(a)\right\|_{H_{\mathrm{o}}^{1}\left((0, \infty), \gamma\left(H, \mathbb{B}^{*}\right)\right)}\left\|G_{\mathbb{B}}^{\lambda, \beta}(f)\right\|_{B M O_{\circ}((0, \infty), \gamma(H, \mathbb{B}))} \\
& \leq C\|a\|_{H_{\mathrm{o}}^{1}\left((0, \infty), \mathbb{B}^{*}\right)}\left\|G_{\mathbb{B}}^{\lambda, \beta}(f)\right\|_{B M O_{\circ}((0, \infty), \gamma(H, \mathbb{B}))} .
\end{aligned}
$$

From (44) it follows that

$$
\|f\|_{B M O_{\circ}((0, \infty), \mathbb{B})} \leq C\left\|G_{\mathbb{B}}^{\lambda, \beta}(f)\right\|_{B M O_{o}((0, \infty), \gamma(H, \mathbb{B}))} .
$$

2.4. Our objective is to prove that, for every $a \in H_{\mathrm{o}}^{1}((0, \infty), \mathbb{B})$,

$$
\|a\|_{H_{\mathrm{o}}^{1}((0, \infty), \mathbb{B})} \leq C\left\|G_{\mathbb{B}}^{\lambda, \beta}(a)\right\|_{H_{\mathrm{o}}^{1}((0, \infty), \gamma(H, \mathbb{B})} .
$$

We have that

$$
\|a\|_{H_{\mathrm{o}}^{1}((0, \infty), \mathbb{B})}=\sup _{\substack{f \in B M O_{\mathrm{o}}\left((0, \infty), \mathbb{B}^{*}\right) \\\|f\|_{B M O_{\mathrm{o}}\left((0, \infty), \mathbb{B}^{*}\right) \leq 1}}}\left|\int_{0}^{\infty}\langle f(x), a(x)\rangle_{\mathbb{B}^{*}, \mathbb{B}} d x\right|, \quad a \in H_{\mathrm{o}}^{1}((0, \infty), \mathbb{B}) .
$$


Suppose that $a \in \mathcal{A} \otimes \mathbb{B}$, where $\mathcal{A}$ is defined as in Section 2.3. By proceeding as in Section 2.3 we get

$$
\begin{aligned}
\left|\int_{0}^{\infty}\langle f(x), a(x)\rangle_{\mathbb{B}^{*}, \mathbb{B}}\right| & \leq C\left\|G_{\mathbb{B}^{*}}^{\lambda, \beta}(f)\right\|_{B M O_{\circ}\left((0, \infty), \gamma\left(H, \mathbb{B}^{*}\right)\right)}\left\|G_{\mathbb{B}}^{\lambda, \beta}(a)\right\|_{H_{\mathrm{o}}^{1}((0, \infty), \gamma(H, \mathbb{B}))} \\
& \leq C\|f\|_{B M O_{\circ}\left((0, \infty), \mathbb{B}^{*}\right)}\left\|G_{\mathbb{B}}^{\lambda, \beta}(a)\right\|_{H_{\mathrm{o}}^{1}((0, \infty), \gamma(H, \mathbb{B}))} .
\end{aligned}
$$

Hence, 46 holds.

In order to see that 46 holds for every $a \in H_{\mathrm{o}}^{1}((0, \infty), \mathbb{B})$ it is enough to take into account that $\mathcal{A} \otimes \mathbb{B}$ is a dense subset of $H_{\mathrm{o}}^{1}((0, \infty), \mathbb{B})$ and that the operator $G_{\mathbb{B}}^{\lambda, \beta}$ is bounded from $H_{\mathrm{o}}^{1}((0, \infty), \mathbb{B})$ into $H_{\mathrm{o}}^{1}((0, \infty), \gamma(H, \mathbb{B}))$ (Section 2.1).

\section{Proof of Theorem 1.2}

In this section we prove that

$$
\mathcal{G}_{\mathbb{B}}^{\lambda}(f)(t, x)=t D_{\lambda, x}^{*} P_{t}^{\lambda+1}(f)(x), \quad t, x \in(0, \infty),
$$

is a bounded operator from $H_{\mathrm{o}}^{1}((0, \infty), \mathbb{B})$ into $H_{\mathrm{o}}^{1}((0, \infty), \gamma(H, \mathbb{B}))$ and from $B M O_{\mathrm{o}}((0, \infty), \mathbb{B})$ into $B M O_{\mathrm{o}}((0, \infty), \gamma(H, \mathbb{B}))$. Here $D_{\lambda, x}^{*}=-x^{-\lambda} \frac{d}{d x} x^{\lambda}$.

3.1. We establish now the behavior of $\mathcal{G}_{\mathbb{B}}^{\lambda}$ between Hardy spaces. In [6] Theorem 1.3] we proved that the operator $\mathcal{G}_{\mathbb{B}}^{\lambda}$ is bounded from $L^{p}((0, \infty), \mathbb{B})$ into $L^{p}((0, \infty), \gamma(H, \mathbb{B}))$, for every $1<p<\infty$.

We show that $\mathcal{G}_{\mathbb{B}}^{\lambda}$ is a $(\mathbb{B}, \gamma(H, \mathbb{B}))$-Calderón-Zygmund operator.

Lemma 3.1. Let $B$ be a UMD Banach space and $\lambda \geq 1$. The operator $\mathcal{G}_{\mathbb{B}}^{\lambda}$ is a $(\mathbb{B}, \gamma(H, \mathbb{B}))$ Calderón-Zygmund operator.

Proof. We consider the function

$$
M^{\lambda}(t ; x, y)=t D_{\lambda, x}^{*} P_{t}^{\lambda+1}(x, y), \quad t, x, y \in(0, \infty) .
$$

$M^{\lambda}$ defines, as it will be specified, a standard $(\mathbb{B}, \gamma(H, \mathbb{B}))$-Calderón-Zygmund kernel. Indeed, we have that

$$
\begin{aligned}
M^{\lambda}(t ; x, y)= & -t x^{-\lambda} \partial_{x}\left[\frac{2(\lambda+1) t}{\pi} x^{2 \lambda+1} y^{\lambda+1} \int_{0}^{\pi} \frac{(\sin \theta)^{2 \lambda+1}}{\left((x-y)^{2}+t^{2}+2 x y(1-\cos \theta)\right)^{\lambda+2}} d \theta\right] \\
= & -\frac{2(\lambda+1)}{\pi} t^{2} y^{\lambda+1}\left[(2 \lambda+1) x^{\lambda} \int_{0}^{\pi} \frac{(\sin \theta)^{2 \lambda+1}}{\left((x-y)^{2}+t^{2}+2 x y(1-\cos \theta)\right)^{\lambda+2}} d \theta\right. \\
& \left.-2(\lambda+2) x^{\lambda+1} \int_{0}^{\pi} \frac{(\sin \theta)^{2 \lambda+1}((x-y)+y(1-\cos \theta))}{\left((x-y)^{2}+t^{2}+2 x y(1-\cos \theta)\right)^{\lambda+3}} d \theta\right], \quad t, x, y \in(0, \infty) .
\end{aligned}
$$

Then,

$$
\begin{aligned}
\left|M^{\lambda}(t ; x, y)\right| \leq & C t^{2}\left[(x y)^{\lambda+1} \int_{0}^{\pi} \frac{(\sin \theta)^{2 \lambda+1}}{(|x-y|+t+\sqrt{2 x y(1-\cos \theta)})^{2 \lambda+5}} d \theta\right. \\
& \left.+y^{\lambda+1} x^{\lambda} \int_{0}^{\pi} \frac{(\sin \theta)^{2 \lambda+1}}{(|x-y|+t+\sqrt{2 x y(1-\cos \theta)})^{2 \lambda+4}} d \theta\right] \\
= & C\left[\mathcal{I}_{1}(t ; x, y)+\mathcal{I}_{2}(t ; x, y)\right], \quad t, x, y \in(0, \infty) .
\end{aligned}
$$

We observe that $\mathcal{I}_{j}(t ; x, y)=t^{2} I_{j}(t / 2, t / 2 ; x, y), t, x, y \in(0, \infty), j=1,2$, where $I_{j}, j=1,2$, are the functions appearing in 12 for $m=1$ and $\lambda+1$ instead of $\lambda$. 
Then, by 13 and 14 , for $j=1,2$, we have that

$$
\mathcal{I}_{j}(t ; x, y) \leq C \frac{t^{2}}{(t+|x-y|)^{3}}, \quad t, x, y \in(0, \infty)
$$

and

Hence,

$$
\left\|\mathcal{I}_{j}(t ; x, y)\right\|_{H} \leq C\left(\int_{0}^{\infty} \frac{t^{3}}{(t+|x-y|)^{6}} d t\right)^{1 / 2}=\frac{C}{|x-y|}, \quad x, y \in(0, \infty), x \neq y .
$$

$$
\left\|M^{\lambda}(\cdot ; x, y)\right\|_{H} \leq \frac{C}{|x-y|}, \quad x, y \in(0, \infty), x \neq y .
$$

We consider, for every $x, y \in(0, \infty), x \neq y$, the operator

$$
\begin{aligned}
M^{\lambda}(x, y): \mathbb{B} & \longrightarrow \gamma(H, \mathbb{B}) \\
b & \longrightarrow M^{\lambda}(x, y)(b): H \longrightarrow \mathbb{B} \\
h & \longrightarrow \int_{0}^{\infty} M^{\lambda}(t ; x, y) h(t) \frac{d t}{t} b .
\end{aligned}
$$

We have that, for every $b \in \mathbb{B}$,

$$
\left\|M^{\lambda}(x, y)(b)\right\|_{\gamma(H, \mathbb{B})} \leq\left\|M^{\lambda}(\cdot ; x, y)\right\|_{H}\|b\|_{\mathbb{B}} \leq C \frac{\|b\|_{\mathbb{B}}}{|x-y|}, \quad x, y \in(0, \infty), x \neq y .
$$

Then,

$$
\left\|M^{\lambda}(x, y)\right\|_{\mathbb{B} \rightarrow \gamma(H, \mathbb{B})} \leq \frac{C}{|x-y|}, \quad x, y \in(0, \infty), x \neq y
$$

We can write, for every $t, x, y \in(0, \infty)$,

$$
\begin{aligned}
\partial_{x} M^{\lambda}(t ; x, y)= & -\frac{2(\lambda+1)}{\pi} t^{2} y^{\lambda+1}\left[(2 \lambda+1) \lambda x^{\lambda-1} \int_{0}^{\pi} \frac{(\sin \theta)^{2 \lambda+1}}{\left((x-y)^{2}+t^{2}+2 x y(1-\cos \theta)\right)^{\lambda+2}} d \theta\right. \\
& -2(2 \lambda+1)(\lambda+2) x^{\lambda} \int_{0}^{\pi} \frac{(\sin \theta)^{2 \lambda+1}((x-y)+y(1-\cos \theta))}{\left((x-y)^{2}+t^{2}+2 x y(1-\cos \theta)\right)^{\lambda+3}} d \theta \\
& -2(\lambda+1)(\lambda+2) x^{\lambda} \int_{0}^{\pi} \frac{(\sin \theta)^{2 \lambda+1}((x-y)+y(1-\cos \theta))}{\left((x-y)^{2}+t^{2}+2 x y(1-\cos \theta)\right)^{\lambda+3}} d \theta \\
& +4(\lambda+2)(\lambda+3) x^{\lambda+1} \int_{0}^{\pi} \frac{(\sin \theta)^{2 \lambda+1}((x-y)+y(1-\cos \theta))^{2}}{\left((x-y)^{2}+t^{2}+2 x y(1-\cos \theta)\right)^{\lambda+4}} d \theta \\
& \left.-2(\lambda+2) x^{\lambda+1} \int_{0}^{\pi} \frac{(\sin \theta)^{2 \lambda+1}}{\left((x-y)^{2}+t^{2}+2 x y(1-\cos \theta)\right)^{\lambda+3}} d \theta\right]
\end{aligned}
$$

It follows that

$$
\begin{aligned}
\left|\partial_{x} M^{\lambda}(t ; x, y)\right| \leq & C t^{2}\left[y^{\lambda+1} x^{\lambda-1} \int_{0}^{\pi} \frac{(\sin \theta)^{2 \lambda+1}}{(|x-y|+t+\sqrt{2 x y(1-\cos \theta)})^{2 \lambda+4}} d \theta\right. \\
& +y^{\lambda+1} x^{\lambda} \int_{0}^{\pi} \frac{(\sin \theta)^{2 \lambda+1}}{(|x-y|+t+\sqrt{2 x y(1-\cos \theta)})^{2 \lambda+5}} d \theta \\
& \left.+(y x)^{\lambda+1} \int_{0}^{\pi} \frac{(\sin \theta)^{2 \lambda+1}}{(|x-y|+t+\sqrt{2 x y(1-\cos \theta)})^{2 \lambda+6}} d \theta\right] \\
= & C\left[J_{1}(t ; x, y)+J_{2}(t ; x, y)+J_{3}(t ; x, y)\right], \quad t, x, y \in(0, \infty) .
\end{aligned}
$$

We note that $J_{2}(t ; x, y)=t^{2} I_{2}(t / 2, t / 2 ; x, y)$ and $J_{3}(t ; x, y)=t^{2} I_{1}(t / 2, t / 2 ; x, y), t, x, y \in(0, \infty)$, being $I_{j}, j=1,2$, the functions in 12 for $m=2$ and $\lambda+1$ instead of $\lambda$. Hence, by (13) and (14), for $\ell=2,3$,

$$
J_{\ell}(t ; x, y) \leq C \frac{t^{2}}{(t+|x-y|)^{4}}, \quad t, x, y \in(0, \infty)
$$


On the other hand, since $\lambda \geq 1$, by proceeding as in the estimation (14), we obtain

$$
J_{1}(t ; x, y) \leq C \frac{t^{2}}{(t+|x-y|)^{4}}, \quad t, x, y \in(0, \infty)
$$

Thus, for $\ell=1,2,3$,

$$
\left\|J_{\ell}(\cdot ;, x, y)\right\|_{H} \leq C\left(\int_{0}^{\infty} \frac{t^{3}}{(t+|x-y|)^{8}} d t\right)^{1 / 2} \leq \frac{C}{|x-y|^{2}}, \quad x, y \in(0, \infty), x \neq y .
$$

Hence,

$$
\left\|\partial_{x} M^{\lambda}(\cdot ; x, y)\right\|_{H} \leq \frac{C}{|x-y|^{2}}, \quad x, y \in(0, \infty), x \neq y
$$

We have also that

$$
\left\|\partial_{y} M^{\lambda}(\cdot ; x, y)\right\|_{H} \leq \frac{C}{|x-y|^{2}}, \quad x, y \in(0, \infty), x \neq y .
$$

Estimations 51, 56 and 57 show that $M^{\lambda}$ is a standard $(\mathbb{B}, \gamma(H, \mathbb{B}))$-Calderón-Zygmund kernel.

Let $f \in S_{\lambda}(0, \infty) \otimes \mathbb{B}$. According to 51 we have that

$$
\int_{0}^{\infty}\left\|M^{\lambda}(x, y)\right\|_{\mathbb{B} \rightarrow \gamma(H, \mathbb{B})}\|f(y)\|_{\mathbb{B}} d y<\infty, \quad x \notin \operatorname{supp} f .
$$

We define

$$
Q^{\lambda}(f)(x)=\int_{0}^{\infty} M^{\lambda}(x, y) f(y) d y, \quad x \notin \operatorname{supp} f,
$$

where the integral is understood in the $\gamma(H, \mathbb{B})$-Bochner sense.

We can differentiate under the integral sign to get

$$
\mathcal{G}_{\mathbb{B}}^{\lambda}(f)(t, x)=\int_{0}^{\infty} M^{\lambda}(t ; x, y) f(y) d y, \quad t, x \in(0, \infty),
$$

and by using Minkowski's inequality and (51) we obtain

$$
\left\|\mathcal{G}_{\mathbb{B}}^{\lambda}(f)(\cdot, x)\right\|_{L^{2}((0, \infty), d t / t, \mathbb{B})} \leq C \int_{0}^{\infty} \frac{\|f(y)\|_{\mathbb{B}}}{|x-y|} d y, \quad x \notin \operatorname{supp} f .
$$

We consider, for every $x \notin \operatorname{supp} f$, the operator

$$
\begin{aligned}
\mathbb{G}_{\mathbb{B}}^{\lambda}(f)(x): H & \longrightarrow \mathbb{B} \\
h & \longrightarrow\left[\mathbb{G}_{\mathbb{B}}^{\lambda}(f)(x)\right](h)=\int_{0}^{\infty} \mathcal{G}_{\mathbb{B}}^{\lambda}(f)(t, x) h(t) \frac{d t}{t} .
\end{aligned}
$$

We can write

$$
\begin{aligned}
{\left[Q^{\lambda}(f)(x)\right](h) } & =\int_{0}^{\infty}\left[M^{\lambda}(x, y) f(y)\right](h) d y \\
& =\int_{0}^{\infty} \int_{0}^{\infty} M^{\lambda}(t ; x, y) h(t) \frac{d t}{t} f(y) d y \\
& =\int_{0}^{\infty} \mathcal{G}_{\mathbb{B}}^{\lambda}(f)(t, x) h(t) \frac{d t}{t}, \quad h \in H \text { and } x \notin \operatorname{supp} f
\end{aligned}
$$

The interchange of the order of integration is justified because

$\int_{0}^{\infty} \int_{0}^{\infty}\left|M^{\lambda}(t ; x, y)\left\|h(t) \mid \frac{d t}{t}\right\| f(y)\left\|_{\mathbb{B}} d y \leq C\right\| h \|_{H} \int_{0}^{\infty} \frac{\|f(y)\|_{\mathbb{B}}}{|x-y|} d y<\infty, \quad h \in H\right.$ and $x \notin \operatorname{supp} f$.

Hence, we deduce that

$$
Q^{\lambda}(f)(x)=\mathcal{G}_{\mathbb{B}}^{\lambda}(f)(\cdot, x), \quad x \notin \operatorname{supp} f
$$

as elements of $\gamma(H, \mathbb{B})$. 
According to [6. Theorem 1.3], by using vector-valued Calderón-Zygmund theory (34]) we infer that the operator defined by 58 can be extended from $S^{\lambda}(0, \infty) \otimes \mathbb{B}$ to $L^{1}((0, \infty), \mathbb{B})$ as a bounded operator from $L^{1}((0, \infty), \mathbb{B})$ into $L^{1, \infty}((0, \infty), \gamma(H, \mathbb{B}))$ and from $H^{1}((0, \infty), \mathbb{B})$ into $L^{1}((0, \infty), \gamma(H, \mathbb{B}))$. By proceeding as in the proof of Lemma 2.1 we can conclude that the operator $\mathcal{G}_{\mathbb{B}}^{\lambda}$ is bounded from $L^{1}((0, \infty), \mathbb{B})$ into $L^{1, \infty}((0, \infty), \gamma(H, \mathbb{B}))$ and from $H^{1}((0, \infty), \mathbb{B})$ into $L^{1}((0, \infty), \gamma(H, \mathbb{B}))$.

By 48 we have that

$$
\left|M^{\lambda}(t ; x, y)\right| \leq C\left[\mathcal{I}_{1}(t ; x, y)+\mathcal{I}_{2}(t ; x, y)\right], \quad t, x, y \in(0, \infty),
$$

where

$$
\begin{aligned}
& \mathcal{I}_{1}(t ; x, y)=t^{2}(x y)^{\lambda+1} \int_{0}^{\pi} \frac{(\sin \theta)^{2 \lambda+1}}{(|x-y|+t+\sqrt{2 x y(1-\cos \theta)})^{2 \lambda+5}} d \theta, \quad t, x, y \in(0, \infty), \\
& \mathcal{I}_{2}(t ; x, y)=t^{2} y^{\lambda+1} x^{\lambda} \int_{0}^{\pi} \frac{(\sin \theta)^{2 \lambda+1}}{(|x-y|+t+\sqrt{2 x y(1-\cos \theta)})^{2 \lambda+4}} d \theta, \quad t, x, y \in(0, \infty),
\end{aligned}
$$

and $M^{\lambda}(t ; x, y), t, x, y \in(0, \infty)$, is the kernel introduced in 477 .

We obtain (see the proof of (7) with $\lambda+1$ instead of $\lambda$ and $k=2$ )

$$
\left|\mathcal{I}_{1}(t ; x, y)\right| \leq C \frac{t^{2}(x y)^{\lambda+1}}{(t+|x-y|)^{2 \lambda+5}}, \quad t, x, y \in(0, \infty)
$$

and

$$
\left|\mathcal{I}_{2}(t ; x, y)\right| \leq C \frac{t^{2} y^{\lambda+1} x^{\lambda}}{(t+|x-y|)^{2 \lambda+4}}, \quad t, x, y \in(0, \infty)
$$

Then,

$$
\left\|\mathcal{I}_{1}(\cdot ; x, y)\right\|_{H} \leq C \frac{(x y)^{\lambda+1}}{|x-y|^{2 \lambda+3}}, \quad x, y \in(0, \infty), x \neq y
$$

and

$$
\left\|\mathcal{I}_{2}(\cdot ; x, y)\right\|_{H} \leq C \frac{y^{\lambda+1} x^{\lambda}}{|x-y|^{2 \lambda+2}}, \quad x, y \in(0, \infty), x \neq y .
$$

Let $\delta>0$ and $b \in \mathbb{B},\|b\|_{\mathbb{B}}=1$. We define $a=\frac{b}{\delta} \chi_{(0, \delta)}$. Since $\mathcal{G}_{\mathbb{B}}^{\lambda}$ is bounded from $L^{2}((0, \infty), \mathbb{B})$ into $L^{2}((0, \infty), \gamma(H, \mathbb{B}))([\underline{6}$, Theorem 1.3]), we deduce that

$$
\int_{0}^{2 \delta}\left\|\mathcal{G}_{\mathbb{B}}^{\lambda}(a)(\cdot, x)\right\|_{\gamma(H, \mathbb{B})} d x \leq C \delta^{1 / 2}\left\|\mathcal{G}_{\mathbb{B}}^{\lambda}(a)\right\|_{L^{2}((0, \infty), \gamma(H, \mathbb{B}))} \leq C \delta^{1 / 2}\|a\|_{L^{2}((0, \infty), \mathbb{B})} \leq C,
$$

where $C>0$ does not depend on $\delta$ or $b$. Also, by (61) and (62), we get

$$
\begin{aligned}
\int_{2 \delta}^{\infty}\left\|\mathcal{G}_{\mathbb{B}}^{\lambda}(a)(\cdot, x)\right\|_{\gamma(H, \mathbb{B})} d x & \leq \frac{C}{\delta} \int_{2 \delta}^{\infty} \int_{0}^{\delta}\left(\frac{y^{\lambda+1} x^{\lambda}}{|x-y|^{2 \lambda+2}}+\frac{(x y)^{\lambda+1}}{|x-y|^{2 \lambda+3}}\right) d y d x \\
& \leq \frac{C}{\delta} \int_{2 \delta}^{\infty} \frac{d x}{x^{\lambda+2}} \int_{0}^{\delta} y^{\lambda+1} d y \leq C,
\end{aligned}
$$

where $C>0$ does not depend on $\delta$ or $b$. We conclude that $\left\|\mathcal{G}_{\mathbb{B}}^{\lambda}(a)\right\|_{L^{1}((0, \infty), \gamma(H, \mathbb{B}))} \leq C$, where $C>0$ does not depend on $\delta$ or $b$.

Also, since $\mathcal{G}_{\mathbb{B}}^{\lambda}$ is bounded from $H^{1}((0, \infty), \mathbb{B})$ into $L^{1}((0, \infty), \gamma(H, \mathbb{B}))$, there exists $C>0$ such that, for every 2-atom $a$ satisfying (Aii),

$$
\left\|\mathcal{G}_{\mathbb{B}}^{\lambda}(a)\right\|_{L^{1}((0, \infty), \gamma(H, \mathbb{B}))} \leq C .
$$

Then, by taking into account that $\mathcal{G}_{\mathbb{B}}^{\lambda}$ is bounded from $L^{1}((0, \infty), \mathbb{B})$ into $L^{1, \infty}((0, \infty), \gamma(H, \mathbb{B}))$, we conclude that $\mathcal{G}_{\mathbb{B}}^{\lambda}$ is bounded from $H_{\mathrm{o}}^{1}((0, \infty), \mathbb{B})$ into $L^{1}((0, \infty), \gamma(H, \mathbb{B}))$. 
Since the Hardy space $H_{\mathrm{o}}^{1}((0, \infty), \mathbb{B})$ does not depend on $\lambda$, in order to see that $\mathcal{G}_{\mathbb{B}}^{\lambda}$ is bounded from $H_{\mathrm{o}}^{1}((0, \infty), \mathbb{B})$ into $H_{\mathrm{o}}^{1}((0, \infty), \gamma(H, \mathbb{B}))$ it is enough to show that $P_{*}^{\lambda+1} \circ \mathcal{G}_{\mathbb{B}}^{\lambda}$ is bounded from $H_{\mathrm{o}}^{1}((0, \infty), \mathbb{B})$ into $L^{1}(0, \infty)$, where

$$
P_{*}^{\lambda+1}(f)=\sup _{s>0}\left\|P_{s}^{\lambda+1}(f)\right\|_{\gamma(H, \mathbb{B})}, \quad f \in L^{p}((0, \infty), \gamma(H, \mathbb{B})), 1 \leq p<\infty .
$$

Since the maximal operator $P_{*}^{\lambda+1}$ is bounded from $L^{1}((0, \infty), \gamma(H, \mathbb{B}))$ into $L^{1, \infty}(0, \infty)$, the operator $P_{*}^{\lambda+1} \circ \mathcal{G}_{\mathbb{B}}^{\lambda}$ is bounded from $H_{\mathrm{o}}^{1}((0, \infty), \mathbb{B})$ into $L^{1, \infty}(0, \infty)$. By using vector-valued Calderón-Zygmund theory as in the proof of Lemma 2.3 we can see that $P_{*}^{\lambda+1} \circ \mathcal{G}_{\mathbb{B}}^{\lambda}$ is bounded from $H^{1}((0, \infty), \mathbb{B})$ into $L^{1}(0, \infty)$. Moreover, as above, we can prove that there exists $C>0$ such that, for every $\delta>0$ and $b \in \mathbb{B},\|b\|_{\mathbb{B}}=1$,

$$
\left\|P_{*}^{\lambda+1}\left(\mathcal{G}_{\mathbb{B}}^{\lambda}(a)\right)\right\|_{L^{1}(0, \infty)} \leq C,
$$

being $a=\frac{b}{\delta} \chi_{(0, \delta)}$. Then, we conclude that $P_{*}^{\lambda+1} \circ \mathcal{G}_{\mathbb{B}}^{\lambda}$ is bounded from $H_{\mathrm{o}}^{1}((0, \infty), \mathbb{B})$ into $L^{1}(0, \infty)$.

Thus the proof of Theorem 1.2 related to Hardy spaces is complete.

3.2. Our objective is to show that $\mathcal{G}_{\mathbb{B}}^{\lambda}$ is bounded from $B M O_{\circ}((0, \infty), \mathbb{B})$ into $B M O_{\circ}((0, \infty), \gamma(H, \mathbb{B}))$. As in Section 2.2 this is naturally divided into the following two lemmas.

Lemma 3.2. Assume that $\mathbb{B}$ is a UMD Banach space and $\lambda>0$. We can find $C>0$ such that, for every $r>0$,

$$
\frac{1}{r} \int_{0}^{r}\left\|\mathcal{G}_{\mathbb{B}}^{\lambda}(f)(\cdot, x)\right\|_{\gamma(H, \mathbb{B})} d x \leq C\|f\|_{B M O_{\circ}((0, \infty), \mathbb{B})}, \quad f \in B M O_{\circ}((0, \infty), \mathbb{B}) .
$$

Proof. Let $f \in B M O_{\circ}((0, \infty), \mathbb{B})$. According to [48], 59 and 60 we get

$$
\left|D_{\lambda, x}^{*} P_{t}^{\lambda+1}(x, y)\right| \leq C t\left(\frac{(x y)^{\lambda+1}}{(|x-y|+t)^{2 \lambda+5}}+\frac{y^{\lambda+1} x^{\lambda}}{(|x-y|+t)^{2 \lambda+4}}\right), \quad t, x, y \in(0, \infty) .
$$

Since $\int_{0}^{\infty}\left(1+y^{2}\right)^{-1}\|f(y)\|_{\mathbb{B}} d y<\infty$, we can write

$$
\mathcal{G}_{\mathbb{B}}^{\lambda}(f)(t, x)=\int_{0}^{\infty} t D_{\lambda, x}^{*} P_{t}^{\lambda+1}(x, y) f(y) d y, \quad t, x \in(0, \infty) .
$$

Let $r>0$. Since $\mathcal{G}_{\mathbb{B}}^{\lambda}$ is bounded from $L^{2}((0, \infty), \mathbb{B})$ into $L^{2}((0, \infty), \gamma(H, \mathbb{B}))$ ([6, Theorem 1.3]) we get

$$
\begin{aligned}
\frac{1}{r} \int_{0}^{r}\left\|\mathcal{G}_{\mathbb{B}}^{\lambda}\left(f \chi_{(0,2 r)}\right)(\cdot, x)\right\|_{\gamma(H, \mathbb{B})} d x & \leq \frac{1}{\sqrt{r}}\left\|\mathcal{G}_{\mathbb{B}}^{\lambda}\left(f \chi_{(0,2 r)}\right)\right\|_{L^{2}((0, \infty), \gamma(H, \mathbb{B}))} \\
& \leq \frac{C}{\sqrt{r}}\left\|f \chi_{(0,2 r)}\right\|_{L^{2}((0, \infty), \mathbb{B})} \leq C\|f\|_{B M O_{o}((0, \infty), \mathbb{B})} .
\end{aligned}
$$

Moreover, by using 61, and 62, as in 31 we obtain

$$
\begin{gathered}
\frac{1}{r} \int_{0}^{r}\left\|\mathcal{G}_{\mathbb{B}}^{\lambda}\left(f \chi_{(2 r, \infty)}\right)(\cdot, x)\right\|_{\gamma(H, \mathbb{B})} d x \leq \frac{1}{r} \int_{0}^{r} \int_{2 r}^{\infty}\left\|M^{\lambda}(\cdot ; x, y)\right\|_{H}\|f(y)\|_{\mathbb{B}} d y d x \\
\leq \frac{1}{r} \int_{0}^{r} \int_{2 r}^{\infty}\left(\frac{(x y)^{\lambda+1}}{|x-y|^{2 \lambda+3}}+\frac{y^{\lambda+1} x^{\lambda}}{|x-y|^{2 \lambda+2}}\right)\|f(y)\|_{\mathbb{B}} d y d x \\
\leq \frac{1}{r} \int_{0}^{r} \int_{2 x}^{\infty} \frac{x^{\lambda}}{y^{\lambda+1}}\|f(y)\|_{\mathbb{B}} d y d x \leq C\|f\|_{B M O_{o}((0, \infty), \mathbb{B}) .}
\end{gathered}
$$

Hence, 63 holds with $C$ independent of $f$ and $r$, and then, $\mathcal{G}_{\mathbb{B}}^{\lambda}(f)(\cdot, x) \in \gamma(H, \mathbb{B})$, a.e. $x \in$ $(0, \infty)$ 
Lemma 3.3. Let $\mathbb{B}$ be a UMD Banach space and $\lambda>0$. $\mathcal{G}_{\mathbb{B}}^{\lambda}$ is a bounded operator from $B M O_{\circ}((0, \infty), \mathbb{B})$ into $B M O((0, \infty), \gamma(H, \mathbb{B}))$.

Proof. Let $f \in B M O_{\mathrm{o}}((0, \infty), \mathbb{B})$. We consider the even extension $f_{\mathrm{e}}$ of the function $f$ to $\mathbb{R}$. Note that $f_{\mathrm{e}} \in B M O(\mathbb{R}, \mathbb{B})$.

We define

$$
\mathcal{G}_{\mathbb{B}}\left(f_{\mathrm{e}}\right)(t, x)=-\int_{\mathbb{R}} t \partial_{x} P_{t}(x-y) f_{\mathrm{e}}(y) d y, \quad t \in(0, \infty) \text { and } x \in \mathbb{R},
$$

where $P_{t}(z)=\frac{1}{\pi} \frac{t}{t^{2}+z^{2}}, t \in(0, \infty)$ and $z \in \mathbb{R}$.

We have that

$$
\mathcal{G}_{\mathbb{B}}\left(f_{\mathrm{e}}\right)(t, x)=-\int_{0}^{\infty} t \partial_{x}\left[P_{t}(x-y)+P_{t}(x+y)\right] f(y) d y, \quad t \in(0, \infty) \text { and } x \in \mathbb{R} .
$$

By using mean value theorem we obtain

$$
\begin{aligned}
\left|\partial_{x}\left[P_{t}(x-y)+P_{t}(x+y)\right]\right| & =\frac{2 t}{\pi}\left|\frac{y-x}{\left(t^{2}+(x-y)^{2}\right)^{2}}-\frac{x+y}{\left(t^{2}+(x+y)^{2}\right)^{2}}\right| \\
& \leq C \frac{t x}{(t+|x-y|)^{4}}, \quad t, x, y \in(0, \infty) .
\end{aligned}
$$

We split $\mathcal{G}_{\mathbb{B}}\left(f_{\mathrm{e}}\right)$ as follows:

$$
\begin{aligned}
\mathcal{G}_{\mathbb{B}}\left(f_{\mathrm{e}}\right)(t, x)= & -\int_{0}^{x / 2} t \partial_{x}\left[P_{t}(x-y)+P_{t}(x+y)\right] f(y) d y \\
& -\int_{2 x}^{\infty} t \partial_{x}\left[P_{t}(x-y)+P_{t}(x+y)\right] f(y) d y \\
& -\int_{x / 2}^{2 x} t \partial_{x} P_{t}(x+y) f(y) d y-\int_{x / 2}^{2 x} t \partial_{x} P_{t}(x-y) f(y) d y \\
= & \sum_{j=1}^{4} I_{j}(f)(t, x), \quad t, x \in(0, \infty) .
\end{aligned}
$$

According to 64 we get

$$
\begin{aligned}
\left\|I_{1}(f)(\cdot, x)\right\|_{\gamma(H, \mathbb{B})} & \leq C x \int_{0}^{x / 2}\|f(y)\|_{\mathbb{B}}\left(\int_{0}^{\infty} \frac{t^{3}}{(t+x)^{8}} d t\right)^{1 / 2} d y \\
& \leq \frac{C}{x} \int_{0}^{x / 2}\|f(y)\|_{\mathbb{B}} d y \leq C\left\|_{f}\right\|_{B M O_{\circ}((0, \infty), \mathbb{B})}, \quad x \in(0, \infty),
\end{aligned}
$$

and, as in 35

$$
\begin{aligned}
\left\|I_{2}(f)(\cdot, x)\right\|_{\gamma(H, \mathbb{B})} & \leq C x \int_{2 x}^{\infty}\|f(y)\|_{\mathbb{B}}\left(\int_{0}^{\infty} \frac{t^{3}}{(t+y)^{8}} d t\right)^{1 / 2} d y \\
& \leq C x \int_{2 x}^{\infty} \frac{\|f(y)\|_{\mathbb{B}}}{y^{2}} d y \leq C\|f\|_{B M O_{\circ}((0, \infty), \mathbb{B})}, \quad x \in(0, \infty) .
\end{aligned}
$$

We also have that

$$
\begin{aligned}
& \left\|I_{3}(f)(\cdot, x)\right\|_{\gamma(H, \mathbb{B})} \leq C \int_{x / 2}^{2 x}\left\|t \partial_{x} P_{t}(x+y)\right\|_{H}\|f(y)\|_{\mathbb{B}} d y \\
& \leq C \int_{x / 2}^{2 x}\|f(y)\|_{\mathbb{B}}\left(\int_{0}^{\infty} \frac{t^{3}}{(t+x+y)^{6}} d t\right)^{1 / 2} d y \\
& \leq \frac{C}{x} \int_{x / 2}^{2 x}\|f(y)\|_{\mathbb{B}} d y \leq C\|f\|_{B M O_{\circ}((0, \infty), \mathbb{B})}, \quad x \in(0, \infty) .
\end{aligned}
$$


We are going to show that $\mathcal{G}_{\mathbb{B}}\left(f_{\mathrm{e}}\right) \in B M O((0, \infty), \gamma(H, \mathbb{B}))$. In order to do this we proceed as in Section 2.2. Let $0<r<s<\infty$. We define $I=(r, s)$ and $2 I=\left(x_{I}-2 d_{I}, x_{I}+2 d_{I}\right)$, where $x_{I}=(r+s) / 2$ and $d_{I}=(s-r) / 2$, and we decompose $f_{\mathrm{e}}$ as follows:

$$
f_{\mathrm{e}}=\left(f_{\mathrm{e}}-f_{I}\right) \chi_{2 I}+\left(f_{\mathrm{e}}-f_{I}\right) \chi_{(0, \infty) \backslash 2 I}+f_{I}=f_{1}+f_{2}+f_{3} .
$$

Since $\mathcal{G}_{\mathbb{B}}$ is a bounded operator from $L^{2}(\mathbb{R}, \mathbb{B})$ into $L^{2}(\mathbb{R}, \gamma(H, \mathbb{B}))([28$, Theorem 4.2]) we deduce that

$$
\frac{1}{|I|} \int_{I}\left\|\mathcal{G}_{\mathbb{B}}\left(f_{1}\right)(\cdot, x)\right\|_{\gamma(H, \mathbb{B})} d x \leq C\|f\|_{B M O_{\mathrm{o}}((0, \infty), \mathbb{B})}
$$

and then, $\mathcal{G}_{\mathbb{B}}\left(f_{1}\right)(\cdot, x) \in \gamma(H, \mathbb{B})$, a.e. $x \in I$. Moreover, since $\int_{\mathbb{R}} P_{t}(x-y) d y=1, t \in(0, \infty)$ and $x \in \mathbb{R}, \mathcal{G}_{\mathbb{B}}\left(f_{3}\right)=0$. Finally, we study $\mathcal{G}_{\mathbb{B}}\left(f_{2}\right)$. We have that

$$
\mathcal{G}_{\mathbb{B}}^{\lambda}(f)(t, x)-\mathcal{G}_{\mathbb{B}}\left(f_{\mathrm{e}}\right)(t, x)=\sum_{j=1}^{3} J_{j}^{\lambda}(f)(t, x)-\sum_{j=1}^{3} I_{j}(f)(t, x), \quad t, x \in(0, \infty),
$$

where

$$
\begin{aligned}
& J_{1}^{\lambda}(f)(t, x)=\int_{0}^{x / 2} M^{\lambda}(t ; x, y) f(y) d y, \quad t, x \in(0, \infty), \\
& J_{2}^{\lambda}(f)(t, x)=\int_{2 x}^{\infty} M^{\lambda}(t ; x, y) f(y) d y, \quad t, x \in(0, \infty),
\end{aligned}
$$

and

$$
J_{3}^{\lambda}(f)(t, x)=\int_{x / 2}^{2 x}\left[M^{\lambda}(t ; x, y)+t \partial_{x} P_{t}(x-y)\right] f(y) d y, \quad t, x \in(0, \infty) .
$$

Here, $I_{j}, j=1,2,3$, are defined as in 65 and $M^{\lambda}$ is the kernel in 477.

According to 61) and 62 we get

$$
\left\|M^{\lambda}(\cdot ; x, y)\right\|_{H} \leq C\left(\frac{(x y)^{\lambda+1}}{|x-y|^{2 \lambda+3}}+\frac{y^{\lambda+1} x^{\lambda}}{|x-y|^{2 \lambda+2}}\right), \quad x, y \in(0, \infty), x \neq y .
$$

It follows that, for every $x \in(0, \infty)$

$\left\|J_{1}^{\lambda}(f)(\cdot, x)\right\|_{\gamma(H, \mathbb{B})} \leq \int_{0}^{x / 2}\left\|M^{\lambda}(\cdot ; x, y)\right\|_{H}\|f(y)\|_{\mathbb{B}} d y \leq \frac{C}{x} \int_{0}^{x / 2}\|f(y)\|_{\mathbb{B}} d y \leq C\|f\|_{B M O_{\circ}((0, \infty), \mathbb{B})}$, and, as in 31),

$$
\left\|J_{2}^{\lambda}(f)(\cdot, x)\right\|_{\gamma(H, \mathbb{B})} \leq C x^{\lambda} \int_{2 x}^{\infty} \frac{\|f(y)\|_{\mathbb{B}}}{y^{\lambda+1}} d y \leq C\|f\|_{B M O_{\circ}((0, \infty), \mathbb{B})}, \quad x \in(0, \infty) .
$$

We write

$$
\begin{aligned}
M^{\lambda}(t ; x, y)= & -\frac{2(\lambda+1)}{\pi} t^{2} y^{\lambda+1}\left[(2 \lambda+1) x^{\lambda} \int_{0}^{\pi} \frac{(\sin \theta)^{2 \lambda+1}}{\left((x-y)^{2}+t^{2}+2 x y(1-\cos \theta)\right)^{\lambda+2}} d \theta\right. \\
& \left.-2(\lambda+2) x^{\lambda+1} \int_{0}^{\pi} \frac{(\sin \theta)^{2 \lambda+1}((x-y)+y(1-\cos \theta))}{\left((x-y)^{2}+t^{2}+2 x y(1-\cos \theta)\right)^{\lambda+3}} d \theta\right] \\
= & M_{1}^{\lambda}(t ; x, y)+M_{2}^{\lambda}(t ; x, y), \quad t, x, y \in(0, \infty),
\end{aligned}
$$

where

$$
\begin{aligned}
M_{1}^{\lambda}(t ; x, y)= & -\frac{2(\lambda+1)}{\pi} t^{2} y^{\lambda+1}\left[(2 \lambda+1) x^{\lambda} \int_{0}^{\pi / 2} \frac{(\sin \theta)^{2 \lambda+1}}{\left((x-y)^{2}+t^{2}+2 x y(1-\cos \theta)\right)^{\lambda+2}} d \theta\right. \\
& \left.-2(\lambda+2) x^{\lambda+1} \int_{0}^{\pi / 2} \frac{(\sin \theta)^{2 \lambda+1}((x-y)+y(1-\cos \theta))}{\left((x-y)^{2}+t^{2}+2 x y(1-\cos \theta)\right)^{\lambda+3}} d \theta\right], \quad t, x, y \in(0, \infty) .
\end{aligned}
$$


We have that

$$
\begin{aligned}
\left\|M_{2}^{\lambda}(\cdot ; x, y)\right\|_{H} \leq & C\left[x^{2 \lambda+1}\left(\int_{0}^{\infty} \frac{t^{3}}{(|x-y|+t+\sqrt{x y})^{4(\lambda+2)}} d t\right)^{1 / 2}\right. \\
& \left.+x^{2 \lambda+2}\left(\int_{0}^{\infty} \frac{t^{3}}{(|x-y|+t+\sqrt{x y})^{4(\lambda+5 / 2)}} d t\right)^{1 / 2}\right] \leq \frac{C}{x}, \quad 0<\frac{x}{2}<y<2 x
\end{aligned}
$$

We now write

$$
\begin{aligned}
M_{1}^{\lambda}(t ; x, y)= & -\frac{2(\lambda+1)}{\pi} t^{2} y^{\lambda+1}\left[(2 \lambda+1) x^{\lambda} \int_{0}^{\pi / 2} \frac{(\sin \theta)^{2 \lambda+1}}{\left((x-y)^{2}+t^{2}+2 x y(1-\cos \theta)\right)^{\lambda+2}} d \theta\right. \\
& -2(\lambda+2) x^{\lambda+1} \int_{0}^{\pi / 2} \frac{(\sin \theta)^{2 \lambda+1} y(1-\cos \theta)}{\left((x-y)^{2}+t^{2}+2 x y(1-\cos \theta)\right)^{\lambda+3}} d \theta \\
& \left.-2(\lambda+2) x^{\lambda+1} \int_{0}^{\pi / 2} \frac{(x-y)(\sin \theta)^{2 \lambda+1}}{\left((x-y)^{2}+t^{2}+2 x y(1-\cos \theta)\right)^{\lambda+3}} d \theta\right] \\
= & \sum_{j=1}^{3} M_{1, j}^{\lambda}(t ; x, y), \quad t, x, y \in(0, \infty),
\end{aligned}
$$

We obtain that

$$
\left|M_{1,2}^{\lambda}(t ; x, y)\right| \leq C\left|M_{1,1}^{\lambda}(t ; x, y)\right| \leq C t^{2} x^{\lambda} y^{\lambda+1} \int_{0}^{\pi / 2} \frac{\theta^{2 \lambda+1}}{(|x-y|+t+\sqrt{x y} \theta)^{2 \lambda+4}} d \theta, \quad t, x, y \in(0, \infty)
$$

Then,

$$
\begin{aligned}
\left\|M_{1,2}^{\lambda}(\cdot ; x, y)\right\|_{H} & \leq C\left\|M_{1,1}^{\lambda}(t, x, y)\right\|_{H} \leq C x^{\lambda} y^{\lambda+1} \int_{0}^{\pi / 2} \theta^{2 \lambda+1}\left(\int_{0}^{\infty} \frac{t^{3}}{(|x-y|+t+\sqrt{x y} \theta)^{4 \lambda+8}} d t\right)^{1 / 2} d \theta \\
& \leq C x^{\lambda} y^{\lambda+1} \int_{0}^{\pi / 2} \frac{\theta^{2 \lambda+1}}{(|x-y|+\sqrt{x y} \theta)^{2 \lambda+2}} d \theta \\
(68) & \leq C \sqrt{\frac{y}{x}} \int_{0}^{\pi / 2} \frac{d \theta}{|x-y|+\sqrt{x y} \theta} \leq \frac{C}{x} \log \left(1+\frac{\sqrt{x y}}{|x-y|}\right), \quad x, y \in(0, \infty) .
\end{aligned}
$$

We put

$$
J_{3}^{\lambda}(f)=M_{1,1}^{\lambda}(f)+M_{1,2}^{\lambda}(f)+M_{2}^{\lambda}(f)+H^{\lambda}(f)
$$

being

$$
\begin{array}{r}
M_{1, j}^{\lambda}(f)(t, x)=\int_{x / 2}^{2 x} M_{1, j}^{\lambda}(t ; x, y) f(y) d y, \quad t, x \in(0, \infty), j=1,2, \\
M_{2}^{\lambda}(f)(t, x)=\int_{x / 2}^{2 x} M_{2}^{\lambda}(t ; x, y) f(y) d y, \quad t, x \in(0, \infty),
\end{array}
$$

and

$$
H^{\lambda}(f)(t, x)=\int_{x / 2}^{2 x}\left[M_{1,3}^{\lambda}(t ; x, y)+t \partial_{x} P_{t}(x-y)\right] f(y) d y, \quad t, x \in(0, \infty) .
$$

According to 67 we obtain that

$$
\begin{aligned}
\left\|M_{2}^{\lambda}(f)(x)\right\|_{\gamma(H, \mathbb{B})} & \leq C \int_{x / 2}^{2 x}\left\|M_{2}^{\lambda}(\cdot ; x, y)\right\|_{H}\|f(y)\|_{\mathbb{B}} d y \\
& \leq \frac{C}{x} \int_{x / 2}^{2 x}\|f(y)\|_{\mathbb{B}} d y \leq C\|f\|_{B M O_{\circ}((0, \infty), \mathbb{B})}, \quad x \in(0, \infty) .
\end{aligned}
$$


Also, by taking into account 68) we can write

$$
\begin{aligned}
\left\|M_{1, j}^{\lambda}(f)(\cdot, x)\right\|_{\gamma(H, \mathbb{B})} & \leq \frac{C}{x} \int_{x / 2}^{2 x} \log \left(1+\frac{\sqrt{x y}}{|x-y|}\right)\|f(y)\|_{\mathbb{B}} d y \\
& \leq C\left(\frac{1}{x} \int_{x / 2}^{2 x}\left(\log \left(1+\frac{\sqrt{x y}}{|x-y|}\right)\right)^{2} d y\right)^{1 / 2}\left(\frac{1}{x} \int_{x / 2}^{2 x}\|f(y)\|_{\mathbb{B}}^{2} d y\right)^{1 / 2} \\
& \leq C\left(\int_{1 / 2}^{2}\left(\log \left(1+\frac{\sqrt{u}}{|1-u|}\right)\right)^{2} d u\right)^{1 / 2}\|f\|_{B M O_{\circ}((0, \infty), \mathbb{B})} \\
& \leq C\|f\|_{B M O_{\circ}((0, \infty), \mathbb{B})}, \quad x \in(0, \infty) \text { and } j=1,2 .
\end{aligned}
$$

We now consider

$$
H^{\lambda}(t ; x, y)=M_{1,3}^{\lambda}(t ; x, y)+t \partial_{x} P_{t}(x-y), \quad t, x, y \in(0, \infty)
$$

We have that

$$
\begin{aligned}
& H^{\lambda}(t ; x, y)=-\frac{2 t^{2}}{\pi} \frac{x-y}{\left(t^{2}+(x-y)^{2}\right)^{2}}+\frac{4(\lambda+1)(\lambda+2)}{\pi} t^{2}(x y)^{\lambda+1} \int_{0}^{\pi / 2} \frac{(x-y)(\sin \theta)^{2 \lambda+1}}{\left((x-y)^{2}+t^{2}+2 x y(1-\cos \theta)\right)^{\lambda+3}} d \theta \\
& =-\frac{2 t^{2}}{\pi}(x-y)\left[\frac{1}{\left(t^{2}+(x-y)^{2}\right)^{2}}-2(\lambda+1)(\lambda+2)(x y)^{\lambda+1} \int_{0}^{\pi / 2} \frac{\theta^{2 \lambda+1}}{\left((x-y)^{2}+t^{2}+x y \theta^{2}\right)^{\lambda+3}} d \theta\right. \\
& \quad+2(\lambda+1)(\lambda+2)(x y)^{\lambda+1} \int_{0}^{\pi / 2} \theta^{2 \lambda+1}\left(\frac{1}{\left((x-y)^{2}+t^{2}+x y \theta^{2}\right)^{\lambda+3}}-\frac{1}{\left((x-y)^{2}+t^{2}+2 x y(1-\cos \theta)\right)^{\lambda+3}}\right) d \theta \\
& \left.\quad+2(\lambda+1)(\lambda+2)(x y)^{\lambda+1} \int_{0}^{\pi / 2} \frac{\theta^{2 \lambda+1}-(\sin \theta)^{2 \lambda+1}}{\left((x-y)^{2}+t^{2}+2 x y(1-\cos \theta)\right)^{\lambda+3}} d \theta\right] \\
& =\sum_{j=1}^{3} H_{j}^{\lambda}(t, x, y), \quad t, x, y \in(0, \infty) .
\end{aligned}
$$

By using mean value theorem we get

$$
\begin{aligned}
\left|H_{2}^{\lambda}(t ; x, y)\right| & \leq C t^{2}|x-y|(x y)^{\lambda+1} \int_{0}^{\pi / 2} \frac{\theta^{2 \lambda+1} x y\left|1-\cos \theta-\theta^{2} / 2\right|}{\left((x-y)^{2}+t^{2}+x y \theta^{2}\right)^{\lambda+4}} d \theta \\
& \leq C t^{2}|x-y|(x y)^{\lambda+2} \int_{0}^{\pi / 2} \frac{\theta^{2 \lambda+5}}{(|x-y|+t+\sqrt{x y} \theta)^{2 \lambda+8}} d \theta \\
& \leq C t^{2}|x-y|(x y)^{\lambda+1} \int_{0}^{\pi / 2} \frac{\theta^{2 \lambda+3}}{(|x-y|+t+\sqrt{x y} \theta)^{2 \lambda+6}} d \theta, \quad t, x, y \in(0, \infty),
\end{aligned}
$$

and

$$
\left|H_{3}^{\lambda}(t ; x, y)\right| \leq C t^{2}|x-y|(x y)^{\lambda+1} \int_{0}^{\pi / 2} \frac{\theta^{2 \lambda+3}}{(|x-y|+t+\sqrt{x y} \theta)^{2 \lambda+6}} d \theta, \quad t, x, y \in(0, \infty) .
$$

Then

$$
\begin{aligned}
\left\|H_{j}^{\lambda}(t ; x, y)\right\|_{H} & \leq C(x y)^{\lambda+1} \int_{0}^{\infty} \theta^{2 \lambda+3}\left(\int_{0}^{\infty} \frac{t^{3}}{(|x-y|+t+\sqrt{x y} \theta)^{4 \lambda+10}} d t\right)^{1 / 2} d \theta \\
& \leq C(x y)^{\lambda+1} \int_{0}^{\pi / 2} \frac{\theta^{2 \lambda+3}}{(|x-y|+\sqrt{x y} \theta)^{2 \lambda+3}} d \theta \\
& \leq C \frac{(x y)^{\lambda+1}}{(\sqrt{x y})^{2 \lambda+3}} \leq \frac{C}{x}, \quad 0<\frac{x}{2}<y<2 x, j=2,3 .
\end{aligned}
$$


On the other hand, after straightforward change of variables we get

$$
\begin{aligned}
H_{1}^{\lambda}(t ; x, y)= & -\frac{2 t^{2}}{\pi}(x-y)\left[\frac{1}{\left(t^{2}+(x-y)^{2}\right)^{2}}-2(\lambda+1)(\lambda+2)(x y)^{\lambda+1}\right. \\
& \left.\times\left(\int_{0}^{\infty}-\int_{\pi / 2}^{\infty}\right) \frac{\theta^{2 \lambda+1}}{\left((x-y)^{2}+t^{2}+x y \theta^{2}\right)^{\lambda+3}} d \theta\right] \\
= & -\frac{2 t^{2}}{\pi}(x-y)\left[\frac{1}{\left(t^{2}+(x-y)^{2}\right)^{2}}\left(1-2(\lambda+1)(\lambda+2) \int_{0}^{\infty} \frac{u^{2 \lambda+1}}{\left(1+u^{2}\right)^{\lambda+3}} d u\right)\right. \\
& \left.+2(\lambda+1)(\lambda+2)(x y)^{\lambda+1} \int_{\pi / 2}^{\infty} \frac{\theta^{2 \lambda+1}}{\left((x-y)^{2}+t^{2}+x y \theta^{2}\right)^{\lambda+3}} d \theta\right] \\
= & -\frac{4(\lambda+1)(\lambda+2)}{\pi} t^{2}(x-y)(x y)^{\lambda+1} \int_{\pi / 2}^{\infty} \frac{\theta^{2 \lambda+1}}{\left((x-y)^{2}+t^{2}+x y \theta^{2}\right)^{\lambda+3}} d \theta, \quad t, x, y \in(0, \infty) .
\end{aligned}
$$

It follows that

$$
\begin{aligned}
\left\|H_{1}^{\lambda}(t ; x, y)\right\|_{H} & \leq C(x y)^{\lambda+1} \int_{\pi / 2}^{\infty} \theta^{2 \lambda+1}\left(\int_{0}^{\infty} \frac{t^{3}}{(|x-y|+t+\sqrt{x y} \theta)^{4 \lambda+10}} d t\right)^{1 / 2} d \theta \\
& \leq C(x y)^{\lambda+1} \int_{\pi / 2}^{\infty} \frac{\theta^{2 \lambda+1}}{(|x-y|+\sqrt{x y} \theta)^{2 \lambda+3}} d \theta \\
& \leq C \frac{(x y)^{\lambda+1}}{(\sqrt{x y})^{2 \lambda+3}} \int_{\pi / 2}^{\infty} \frac{d \theta}{\theta^{2}} \leq \frac{C}{x}, \quad 0<\frac{x}{2}<y<2 x<\infty .
\end{aligned}
$$

We conclude that

$$
\left\|H^{\lambda}(\cdot ; x, y)\right\|_{H} \leq \frac{C}{x}, \quad 0<\frac{x}{2}<y<2 x<\infty,
$$

and hence, for each $x \in(0, \infty)$,

$$
\left\|H^{\lambda}(f)(\cdot, x)\right\|_{\gamma(H, \mathbb{B})} \leq C \int_{x / 2}^{2 x}\left\|H^{\lambda}(\cdot ; x, y)\right\|_{H}\|f(y)\|_{\mathbb{B}} d y \leq \frac{C}{x} \int_{0}^{2 x}\|f(y)\|_{\mathbb{B}} d y \leq C\|f\|_{B M O_{\circ}((0, \infty), \mathbb{B})} .
$$

By putting together the above estimates we obtain that

$$
\left\|\mathcal{G}_{\mathbb{B}}\left(f_{\mathrm{e}}\right)(\cdot, x)-\mathcal{G}_{\mathbb{B}}^{\lambda}(f)(\cdot, x)\right\|_{\gamma(H, \mathbb{B})} \leq C\|f\|_{B M O_{\circ}((0, \infty), \mathbb{B})}, \quad x \in(0, \infty) .
$$

Since $\mathcal{G}_{\mathbb{B}}\left(f_{1}\right)(\cdot, x) \in \gamma(H, \mathbb{B})$, a.e. $\quad x \in(0, \infty), \mathcal{G}_{\mathbb{B}}^{\lambda}(f)(\cdot, x) \in \gamma(H, \mathbb{B})$, a.e. $\quad x \in(0, \infty)$, and $\mathcal{G}_{\mathbb{B}}\left(f_{3}\right)(t, x)=0, t, x \in(0, \infty)$, we deduce that $\mathcal{G}_{\mathbb{B}}\left(f_{2}\right)(\cdot, x) \in \gamma(H, \mathbb{B})$, a.e. $x \in(0, \infty)$.

On the other hand,

$$
\left\|\partial_{x}\left[t \partial_{x} P_{t}(x-y)\right]\right\|_{H} \leq C\left(\int_{0}^{\infty} \frac{t^{3}}{(t+|x-y|)^{8}} d t\right)^{1 / 2} \leq \frac{C}{|x-y|^{2}}, \quad x, y \in(0, \infty), x \neq y .
$$

We have established all the properties needed in order to show, by using the arguments developed in Section 2.2, that

$$
\frac{1}{|I|} \int_{I}\left\|\mathcal{G}_{\mathbb{B}}\left(f_{2}\right)(\cdot, x)-\mathcal{G}_{\mathbb{B}}\left(f_{2}\right)\left(\cdot, x_{0}\right)\right\|_{\mathbb{B}} d x \leq C\|f\|_{B M O_{\circ}((0, \infty), \mathbb{B})},
$$

where $x_{0} \in I$ is chosen such that $\mathcal{G}_{\mathbb{B}}\left(f_{2}\right)\left(\cdot, x_{0}\right) \in \gamma(H, \mathbb{B})$ and the constant $C>0$ does not depend on $I$.

From 66, 69 and 6 we deduce that there exists $C>0$ such that, for every interval $I \subset(0, \infty)$, we can find $\alpha_{I} \in \gamma(H, \mathbb{B})$ such that

$$
\frac{1}{|I|} \int_{I}\left\|\mathcal{G}_{\mathbb{B}}^{\lambda}(f)(\cdot, x)-\alpha_{I}\right\|_{\gamma(H, \mathbb{B})} d x \leq C\|f\|_{B M O_{\circ}((0, \infty), \mathbb{B})}
$$




\section{Proof of Theorem 1.3}

Properties $(i) \Longrightarrow(i i)$ and $(i) \Longrightarrow($ iii) have been established in Theorem 1.1 and Theorem 1.2

4.1. We now prove that $(i i) \Longrightarrow(i)$. In order to do this we use Riesz transfoms in the Bessel setting. The Riesz transform $R_{\lambda}$ is defined by

$$
R_{\lambda}(f)(x)=\lim _{\varepsilon \rightarrow 0^{+}} \int_{0,|x-y|>\varepsilon}^{\infty} R_{\lambda}(x, y) f(y) d y, \text { a.e. } x \in(0, \infty),
$$

for every $f \in L^{p}(0, \infty), 1 \leq p<\infty$. Here the kernel $R_{\lambda}$ is given by

$$
R_{\lambda}(x, y)=\int_{0}^{\infty} D_{\lambda, x} P_{t}^{\lambda}(x, y) d t, \quad x, y \in(0, \infty), x \neq y .
$$

$R_{\lambda}$ is a Calderón-Zygmund operator that is bounded from $L^{p}(0, \infty)$ into itself and from $L^{1}(0, \infty)$ into $L^{1, \infty}(0, \infty)$ ([2, Theorem 4.2]).

We define the Riesz transform on $L^{p}(0, \infty) \otimes \mathbb{B}, 1 \leq p<\infty$, in the natural way, and the new operator is also denoted by $R_{\lambda}$. In [13, Theorem 2.1] it was proved that the Banach space $\mathbb{B}$ is UMD if, and only if, $R_{\lambda}$ can be extended from $L^{p}(0, \infty) \otimes \mathbb{B}$ to $L^{p}((0, \infty), \mathbb{B})$ as a bounded operator from $L^{p}((0, \infty), \mathbb{B})$ into itself, for some (equivalently, for any) $1<p<\infty$.

We consider the operator $R_{\lambda}^{*}$ defined by

$$
R_{\lambda}^{*}(f)(x)=\lim _{\varepsilon \rightarrow 0^{+}} \int_{0,|x-y|>\varepsilon}^{\infty} R_{\lambda}(y, x) f(y) d y, \text { a.e. } x \in(0, \infty),
$$

for every $f \in L^{p}(0, \infty), 1 \leq p<\infty$. This operator has the same $L^{p}$-boundedness properties of $R_{\lambda}$. Also, the Banach space $\mathbb{B}$ is UMD if and only if $R_{\lambda}^{*}$ can be extended from $L^{p}(0, \infty) \otimes \mathbb{B}$ to $L^{p}((0, \infty), \mathbb{B})$ as a bounded operator from $L^{p}((0, \infty), \mathbb{B})$ into itself, for some (equivalently, for any) $1<p<\infty$.

In [33, (16.6)] Cauchy-Riemann type equations in the Bessel setting were given. Motivated by these equations and using Hankel transform we can see that

$$
\partial_{t} P_{t}^{\lambda}\left(R_{\lambda}^{*}(f)\right)=D_{\lambda}^{*} P_{t}^{\lambda+1}(f), \quad f \in S_{\lambda}(0, \infty) .
$$

In other words, we have that

$$
G_{\mathbb{C}}^{\lambda, 1}\left(R_{\lambda}^{*}(f)\right)=\mathcal{G}_{\mathbb{C}}^{\lambda}(f), \quad f \in S_{\lambda}(0, \infty) .
$$

Since $S_{\lambda}(0, \infty)$ is a dense subspace of $L^{p}(0, \infty), 1<p<\infty$, by using [6, Theorems 1.2 and 1.3] we obtain

$$
G_{\mathbb{C}}^{\lambda, 1}\left(R_{\lambda}^{*}(f)\right)=\mathcal{G}_{\mathbb{C}}^{\lambda}(f), \quad f \in L^{p}(0, \infty), 1<p<\infty .
$$

Now, we need to know the behaviour of $R_{\lambda}^{*}$ on $H_{\mathrm{o}}^{1}(\mathbb{R})$ and on $B M O_{\mathrm{o}}(\mathbb{R})$.

Proposition 4.1. Let $\lambda>0$. The Riesz transform $R_{\lambda}^{*}$ is a bounded operator from $E(0, \infty)$ into itself, where $E$ denotes $H_{\mathrm{o}}^{1}$ or $B M O_{\mathrm{o}}$.

Proof of Proposition 4.1: the case of $E=H_{\mathrm{o}}^{1}$. Since $R_{\lambda}^{*}$ is a Calderón-Zygmund operator, $R_{\lambda}^{*}$ is bounded from $H^{1}(0, \infty)$ into $L^{1}(0, \infty)$, and there exists $C>0$ such that, for every $\infty$-atom $a$ satisfying the (Aii) property,

$$
\left\|R_{\lambda}^{*}(a)\right\|_{L^{1}(0, \infty)} \leq C .
$$


According to [2, (3.16)],

$$
\left|R_{\lambda}(y, x)\right| \leq C y^{\lambda+1} x^{-\lambda-2}, \quad 0<y<\frac{x}{2}<\infty .
$$

Let $\delta>0$. If $a=\frac{1}{\delta} \chi_{(0, \delta)}$, being $R_{\lambda}^{*}$ bounded from $L^{2}(0, \infty)$ into itself, by 73 we have that

$$
\begin{aligned}
\left\|R_{\lambda}^{*}(a)\right\|_{L^{1}(0, \infty)} & =\int_{0}^{2 \delta}\left|R_{\lambda}^{*}(a)(x)\right| d x+\int_{2 \delta}^{\infty} \int_{0}^{\delta}\left|R_{\lambda}(y, x)\right| \frac{d y}{\delta} d x \\
& \leq\left(\delta \int_{0}^{\infty}\left|R_{\lambda}^{*}(a)(x)\right|^{2} d x\right)^{1 / 2}+\frac{C}{\delta} \int_{2 \delta}^{\infty} \frac{1}{x^{\lambda+2}} \int_{0}^{\delta} y^{\lambda+1} d y d x \\
& \leq C\left(1+\left(\delta \int_{0}^{\delta}|a(x)|^{2} d x\right)^{1 / 2}\right) \leq C .
\end{aligned}
$$

Here $C>0$ does not depend on $\delta$.

Moreover, since $R_{\lambda}^{*}$ is a bounded operator from $L^{1}(0, \infty)$ into $L^{1, \infty}(0, \infty)$ we obtain that $R_{\lambda}^{*}$ is bounded from $H_{\mathrm{o}}^{1}(0, \infty)$ into $L^{1}(0, \infty)$.

On the other hand, it can be seen that $R_{\lambda}(f)=h_{\lambda+1}\left(h_{\lambda}(f)\right), f \in L^{2}(0, \infty)([33$, §16]). Then,

$$
R_{\lambda}^{*}(f)=h_{\lambda}\left(h_{\lambda+1}(f)\right), \quad f \in L^{2}(0, \infty) .
$$

Then, by using Hankel transforms we get, for every $f \in L^{2}(0, \infty)$,

$$
P_{s}^{\lambda}\left(R_{\lambda}^{*}(f)\right)=h_{\lambda}\left(e^{-s y} h_{\lambda+1}(f)\right)=h_{\lambda} h_{\lambda+1}\left(P_{s}^{\lambda+1}(f)\right)=R_{\lambda}^{*}\left(P_{s}^{\lambda+1}(f)\right), \quad s \in(0, \infty) .
$$

We consider the operators

$$
\mathcal{H}_{*}^{\lambda}(f)(x)=\sup _{s>0}\left|P_{s}^{\lambda}\left(R_{\lambda}^{*}(f)\right)(x)\right|, \quad x \in(0, \infty),
$$

and, for every $N \in \mathbb{N}$,

$$
\mathcal{H}_{N, *}^{\lambda}(f)(x)=\sup _{s \in[1 / N, N]}\left|P_{s}^{\lambda}\left(R_{\lambda}^{*}(f)\right)(x)\right|, \quad x \in(0, \infty),
$$

where $f \in L^{p}(0, \infty), 1<p<\infty . \mathcal{H}_{*}^{\lambda}$ and $\mathcal{H}_{N, *}^{\lambda}, N \in \mathbb{N}$, are bounded operators from $L^{p}(0, \infty)$ into itself, for each $1<p<\infty$.

Since $D_{\lambda, y} P_{t}^{\lambda}(x, y)=-D_{\lambda, x}^{*} P_{t}^{\lambda+1}(x, y), t, x, y \in(0, \infty)$, 74 leads to

$$
P_{s}^{\lambda}\left(R_{\lambda}^{*}(f)\right)(x)=\int_{0}^{\infty} \mathcal{H}^{\lambda}(s ; x, y) f(y) d y, \quad \text { a.e. } x \notin \operatorname{supp} f
$$

for every $f \in L^{2}(0, \infty)$, where

$$
\mathcal{H}^{\lambda}(s ; x, y)=-\int_{0}^{\infty} D_{\lambda, x}^{*} P_{t+s}^{\lambda+1}(x, y) d t, \quad s, x, y \in(0, \infty) .
$$

By 48 and 49 wave that

$$
\begin{aligned}
\int_{0}^{\infty}\left|D_{\lambda, x}^{*} P_{t+s}^{\lambda+1}(x, y)\right| d t & \leq C \int_{0}^{\infty} \frac{t+s}{(t+s+|x-y|)^{3}} d t \leq C \int_{0}^{\infty} \frac{d t}{(t+s+|x-y|)^{2}} \\
& \leq \frac{C}{s+|x-y|}, \quad s, x, y \in(0, \infty)
\end{aligned}
$$

Then

$$
\left\|\mathcal{H}^{\lambda}(\cdot ; x, y)\right\|_{L^{\infty}(0, \infty)} \leq \frac{C}{|x-y|}, \quad x, y \in(0, \infty), x \neq y .
$$

On the other hand, from (52), 53) and (54) it follows that

$$
\int_{0}^{\infty}\left|\partial_{x} D_{\lambda, x}^{*} P_{t+s}^{\lambda+1}(x, y)\right| \leq C \int_{0}^{\infty} \frac{t+s}{(t+s+|x-y|)^{4}} d t \leq \frac{C}{(s+|x-y|)^{2}}, \quad s, x, y \in(0, \infty) .
$$


Hence,

$$
\left\|\partial_{x} \mathcal{H}^{\lambda}(\cdot ; x, y)\right\|_{L^{\infty}(0, \infty)} \leq \frac{C}{|x-y|^{2}}, \quad x, y \in(0, \infty), x \neq y .
$$

The same arguments lead to

$$
\left\|\partial_{y} \mathcal{H}^{\lambda}(\cdot ; x, y)\right\|_{L^{\infty}(0, \infty)} \leq \frac{C}{|x-y|^{2}}, \quad x, y \in(0, \infty), x \neq y .
$$

Let $N \in \mathbb{N}$. We consider the operator

$$
\mathcal{H}_{N}^{\lambda}(f)(s, x)=P_{s}^{\lambda}\left(R_{\lambda}^{*}(f)\right)(x), \quad x \in(0, \infty) \text { and } s \in\left[\frac{1}{N}, N\right] .
$$

For every $f \in S_{\lambda}(0, \infty)$, we have that

$$
\mathcal{H}_{N}^{\lambda}(f)(\cdot, x)=\int_{0}^{\infty} \mathcal{H}_{\lambda}(\cdot ; x, y) f(y) d y, \quad \text { a.e. } x \notin \operatorname{supp} f,
$$

where the integral is understood in the $C[1 / N, N]$-Bochner sense, the space of continuous functions in $[1 / N, N]$. Indeed, let $f \in S_{\lambda}(0, \infty)$. According to $[76$, the integral in 79 is convergent in the $C[1 / N, N]$-Bochner sense, for almost all $x \notin \operatorname{supp} f$. Assume that $\mu \in \mathcal{M}([1 / N, N])$, the space of complex measures supported on $[1 / N, N]$. Since $\mathcal{M}([1 / N, N])=(C[1 / N, N])^{*}$, we can write

$$
\begin{aligned}
\int_{0}^{\infty}\left(\int_{0}^{\infty} \mathcal{H}^{\lambda}(\cdot ; x, y) f(y) d y\right) d \mu(s) & =\int_{0}^{\infty} \int_{0}^{\infty} \mathcal{H}^{\lambda}(s ; x, y) d \mu(s) f(y) d y \\
& =\int_{0}^{\infty}\left(\int_{0}^{\infty} \mathcal{H}^{\lambda}(s ; x, y) f(y) d y\right) d \mu(s), \quad x \notin \operatorname{supp} f
\end{aligned}
$$

Note that the interchange of the order of integration is justified by 76 .

According to the vector-valued Calderón-Zygmund theory, since $\mathcal{H}_{*}^{\lambda}$ is bounded from $L^{2}(0, \infty)$ into itself, estimations (76, 77) and 78 imply that the operator $\mathcal{H}_{N}^{\lambda}$ can be extended to $L^{1}(0, \infty)$ as a bounded operator from $L^{1}(0, \infty)$ into $L^{1, \infty}((0, \infty), C[1 / N, N])$ and from $H^{1}(0, \infty)$ into $L^{1}((0, \infty), C[1 / N, N])$. Moreover, if we denote by $\widetilde{\mathcal{H}}_{N}^{\lambda}$ to this extension we have that

$$
\sup _{N \in \mathbb{N}}\left\|\widetilde{\mathcal{H}}_{N}^{\lambda}\right\|_{L^{1}(0, \infty) \rightarrow L^{1, \infty}((0, \infty), C[1 / N, N])}<\infty
$$

and

$$
\sup _{N \in \mathbb{N}}\left\|\widetilde{\mathcal{H}}_{N}^{\lambda}\right\|_{H^{1}(0, \infty) \rightarrow L^{1}((0, \infty), C[1 / N, N])}<\infty .
$$

We now show that $\widetilde{\mathcal{H}}_{N}^{\lambda}(f)=\mathcal{H}_{N}^{\lambda}(f), f \in L^{1}(0, \infty)$. Indeed, let $f \in L^{1}(0, \infty)$. We choose a sequence $\left(f_{n}\right)_{n \in \mathbb{N}} \subset S_{\lambda}(0, \infty)$ such that $f_{n} \longrightarrow f$, as $n \rightarrow \infty$, in $L^{1}(0, \infty)$. Then

$$
\mathcal{H}_{N}^{\lambda}\left(f_{n}\right) \longrightarrow \widetilde{\mathcal{H}}_{N}^{\lambda}(f), \text { as } n \rightarrow \infty,
$$

in $L^{1, \infty}((0, \infty), C[1 / N, N])$. There exists an increasing sequence $\left(n_{k}\right)_{k \in \mathbb{N}}$ such that

$$
\left\|\mathcal{H}_{N}^{\lambda}\left(f_{n_{k}}\right)(\cdot, x)-\widetilde{\mathcal{H}}_{N}^{\lambda}(f)(x)\right\|_{C[1 / N, N]} \longrightarrow 0, \text { as } k \rightarrow \infty,
$$

for almost all $x \in(0, \infty)$. Moreover by 75$)$,

$$
\mathcal{H}_{N}^{\lambda}\left(f_{n_{k}}\right)(s, x) \longrightarrow \mathcal{H}_{N}^{\lambda}(f)(s, x), \text { as } k \rightarrow \infty, \quad s, x \in(0, \infty) .
$$

Hence, $\mathcal{H}_{N}^{\lambda}(f)(s, x)=\left[\widetilde{\mathcal{H}}_{N}(f)(x)\right](s), s \in[1 / N, N]$ and almost all $x \in(0, \infty)$.

We conclude that $\mathcal{H}_{N}^{\lambda}$ is bounded from $L^{1}(0, \infty)$ into $L^{1, \infty}((0, \infty), C[1 / N, N])$ and from $H^{1}(0, \infty)$ into $L^{1}((0, \infty), C[1 / N, N])$. Moreover

$$
\sup _{N \in \mathbb{N}}\left\|\mathcal{H}_{N}^{\lambda}\right\|_{L^{1}(0, \infty) \rightarrow L^{1, \infty}((0, \infty), C[1 / N, N])}<\infty,
$$


and

$$
\sup _{N \in \mathbb{N}}\left\|\mathcal{H}_{N}^{\lambda}\right\|_{H^{1}(0, \infty) \rightarrow L^{1}((0, \infty), C[1 / N, N])}<\infty .
$$

Then, $\mathcal{H}_{*}^{\lambda}$ is bounded from $L^{1}(0, \infty)$ into $L^{1, \infty}(0, \infty)$ and from $H^{1}(0, \infty)$ into $L^{1}(0, \infty)$.

In order to see that $\mathcal{H}_{*}^{\lambda}$ is bounded from $H_{\mathrm{o}}^{1}(0, \infty)$ into $L^{1}(0, \infty)$ it is enough to show that there exists $C>0$ such that, for every $\infty$-atom $a$,

$$
\left\|\mathcal{H}_{*}^{\lambda}(a)\right\|_{L^{1}(0, \infty)} \leq C
$$

Since $\mathcal{H}_{*}^{\lambda}$ is bounded from $H^{1}(0, \infty)$ into $L^{1}(0, \infty)$, for a certain $C>0,80$ holds provided that $a$ is a an $\infty$-atom satisfying $(A i i)$. Also, by using (48), 59) and (60), and by proceeding as in 75) we deduce that

$$
\left\|\mathcal{H}^{\lambda}(\cdot ; x, y)\right\|_{L^{\infty}(0, \infty)} \leq C(x y)^{\lambda}\left(\frac{x y}{|x-y|^{2 \lambda+3}}+\frac{y}{|x-y|^{2 \lambda+2}}\right) \leq C \frac{y^{\lambda+1}}{x^{\lambda+2}}, \quad 0<y<\frac{x}{2}<\infty .
$$

Also $\mathcal{H}_{*}^{\lambda}$ is bounded from $L^{2}(0, \infty)$ into itself. Then, for every $a=\frac{1}{\delta} \chi_{(0, \delta)}$, with $\delta>0$, by 81 we get

$$
\begin{aligned}
\left\|\mathcal{H}_{*}^{\lambda}(a)\right\|_{L^{1}(0, \infty)} & \leq \int_{0}^{2 \delta}\left|\mathcal{H}_{*}^{\lambda}(a)(x)\right| d x+\int_{2 \delta}^{\infty} \int_{0}^{\delta}\left\|\mathcal{H}^{\lambda}(\cdot ; x, y)\right\|_{L^{\infty}(0, \infty)}|a(y)| d y d x \\
& \leq C\left(\delta^{1 / 2}\|a\|_{L^{2}(0, \infty)}+\frac{1}{\delta} \int_{2 \delta}^{\infty} \frac{1}{x^{\lambda+2}} \int_{0}^{\delta} y^{\lambda+1} d y\right) \leq C,
\end{aligned}
$$

where $C>0$ does not depend on $\delta$.

Hence 80 holds and we obtain that $\mathcal{H}_{*}^{\lambda}$ is bounded from $H_{\mathrm{o}}^{1}(0, \infty)$ into $L^{1}(0, \infty)$. We conclude that $R_{\lambda}^{*}$ is bounded from $H_{\mathrm{o}}^{1}(0, \infty)$ into itself.

Proof of Proposition 4.1; the case of $E=B M O_{\mathrm{o}}(0, \infty)$. Suppose that $f \in B M O_{\mathrm{o}}(0, \infty)$. Let $r>0$. Since $R_{\lambda}^{*}$ is bounded from $L^{2}(0, \infty)$ into itself and that (see [2, (3.15)])

$$
\left|R_{\lambda}(y, x)\right| \leq C \frac{x^{\lambda}}{y^{\lambda+1}}, \quad 0<2 x<y<\infty
$$

we obtain

$$
\begin{aligned}
\frac{1}{r} \int_{0}^{r}\left|R_{\lambda}^{*}(f)(x)\right| d x & \leq \frac{1}{r}\left(\int_{0}^{r}\left|R_{\lambda}^{*}\left(f \chi_{(0,2 r)}\right)(x)\right| d x+\int_{0}^{r}\left|R_{\lambda}^{*}\left(f \chi_{(2 r, \infty)}\right)(x)\right| d x\right) \\
& \leq C\left[\left(\frac{1}{r} \int_{0}^{r}\left|R_{\lambda}^{*}\left(f \chi_{(0,2 r)}\right)(x)\right|^{2} d x\right)^{1 / 2}+\frac{1}{r} \int_{0}^{r} \int_{2 r}^{\infty}\left|R_{\lambda}(y, x)\right||f(y)| d y d x\right] \\
& \leq C\left[\left(\frac{1}{r} \int_{0}^{2 r}|f(y)|^{2} d y\right)^{1 / 2}+r^{\lambda} \int_{2 r}^{\infty} \frac{|f(y)|}{y^{\lambda+1}} d y\right] \\
& \leq C\|f\|_{B M O_{\circ}(0, \infty)}
\end{aligned}
$$

where $C>0$ does not depend on $r$.

The next step is to show that $R_{\lambda}^{*}(f) \in B M O(0, \infty)$.

We consider the even extension $f_{\mathrm{e}}$ of the function $f$ to $\mathbb{R}$. Then, $f_{\mathrm{e}} \in B M O(\mathbb{R})$. According to [40, p. 294] the Hilbert transform $\mathfrak{H}\left(f_{\mathrm{e}}\right)$ given by

$$
\mathfrak{H}\left(f_{e}\right)(x)=\lim _{\varepsilon \rightarrow 0^{+}} \frac{1}{\pi} \int_{|x-y|>\varepsilon}\left(\frac{1}{x-y}-\frac{\chi_{\mathbb{R} \backslash(-1,1)}(y)}{y}\right) f_{e}(y) d y, \quad \text { a.e. } x \in \mathbb{R},
$$

is in $B M O(\mathbb{R})$. 
In [8, (26) and (27)] it was seen that

$$
\mathfrak{H}\left(f_{e}\right)(x)-\lim _{\varepsilon \rightarrow 0^{+}} \frac{1}{\pi} \int_{x / 2,|x-y|>\varepsilon}^{2 x}\left(\frac{1}{x-y}+\frac{\chi_{(1, \infty)}(y)}{y}\right) f(y) d y \in L^{\infty}(0, \infty),
$$

and

(84)

$$
\left\|\mathfrak{H}\left(f_{e}\right)(x)-\lim _{\varepsilon \rightarrow 0^{+}} \frac{1}{\pi} \int_{x / 2,|x-y|>\varepsilon}^{2 x}\left(\frac{1}{x-y}+\frac{\chi_{(1, \infty)}(y)}{y}\right) f(y) d y\right\|_{L^{\infty}(0, \infty)} \leq C\|f\|_{B M O_{\circ}(0, \infty)} .
$$

We now show that

(85)

$$
\left\|R_{\lambda}^{*}(f)(x)+\lim _{\varepsilon \rightarrow 0^{+}} \frac{1}{\pi} \int_{x / 2,|x-y|>\varepsilon}^{2 x}\left(\frac{1}{x-y}+\frac{\chi_{(1, \infty)}(y)}{y}\right) f(y) d y\right\|_{L^{\infty}(0, \infty)} \leq C\|f\|_{B M O_{\circ}(0, \infty)} .
$$

By using [8, (5)], 873) and 83), as in [8, pp. 319 and 320] we deduce that

$$
\begin{aligned}
&\left|R_{\lambda}^{*}(f)(x)+\lim _{\varepsilon \rightarrow 0^{+}} \frac{1}{\pi} \int_{x / 2,|x-y|>\varepsilon}^{2 x}\left[\frac{1}{x-y}+\frac{\chi_{(1, \infty)}(y)}{y}\right) f(y) d y\right| \\
& \leq C {\left[\int_{x / 2}^{2 x} \frac{1}{y}\left(1+\log _{+} \frac{\sqrt{x y}}{|x-y|}\right)|f(y)| d y+\int_{x / 2}^{2 x} \frac{\chi_{(1, \infty)}(y)}{y}|f(y)| d y\right.} \\
&\left.+\frac{1}{x^{\lambda+2}} \int_{0}^{x / 2} y^{\lambda+1}|f(y)| d y+x^{\lambda} \int_{2 x}^{\infty} \frac{|f(y)|}{y^{\lambda+1}} d y\right] \\
& \leq C\left[\left(\int_{x / 2}^{2 x} \frac{1}{y}\left(1+\log _{+} \frac{\sqrt{x y}}{|x-y|}\right)^{2} d y\right)^{1 / 2}\left(\frac{1}{x} \int_{0}^{2 x}|f(y)|^{2} d y\right)^{1 / 2}\right. \\
&\left.+\frac{1}{x} \int_{0}^{2 x}|f(y)| d y+x^{\lambda} \int_{2 x}^{\infty} \frac{|f(y)|}{y^{\lambda+1}} d y\right] \leq C\|f\|_{B M O_{\circ}(0, \infty)}, \quad x \in(0, \infty),
\end{aligned}
$$

where $\log _{+} z=\max \{0, \log z\}, z \in(0, \infty)$. Thus, 85 is established.

From 84 and 85 , since $\mathfrak{H}\left(f_{\mathrm{e}}\right) \in B M O(\mathbb{R})$, we deduce that $R_{\lambda}^{*}(f) \in B M O(0, \infty)$.

Let $f \in L_{c}^{\infty}(0, \infty) \otimes \mathbb{B}$. Since $L_{c}^{\infty}(0, \infty) \otimes \mathbb{B} \subset H_{\mathrm{o}}^{1}(0, \infty) \otimes \mathbb{B}$, by Proposition 4.1. $R_{\lambda}^{*}(f) \in$ $H_{\mathrm{o}}^{1}(0, \infty) \otimes \mathbb{B}$.

Assume that (ii) holds for $H_{\mathrm{o}}^{1}(\mathbb{R}, \mathbb{B})$. By 72 it follows that

$\left\|R_{\lambda}^{*}(f)\right\|_{H_{\mathrm{o}}^{1}((0, \infty), \mathbb{B})} \leq C\left\|G_{\mathbb{B}}^{\lambda, 1}\left(R_{\lambda}^{*}(f)\right)\right\|_{H_{\mathrm{o}}^{1}((0, \infty), \gamma(H, \mathbb{B}))} \leq C\left\|\mathcal{G}_{\mathbb{B}}^{\lambda}(f)\right\|_{H_{\mathrm{o}}^{1}((0, \infty), \gamma(H, \mathbb{B}))} \leq C\|f\|_{H_{\mathrm{o}}^{1}((0, \infty), \mathbb{B})}$.

Since $H^{1}((0, \infty), \mathbb{B}) \subset H_{\mathrm{o}}^{1}((0, \infty), \mathbb{B}) \subset L^{1}((0, \infty), \mathbb{B})$, according to [31, Theorem 4.1], $R_{\lambda}^{*}$ can be extended to $L^{p}((0, \infty), \mathbb{B})$ as a bounded operator from $L^{p}((0, \infty), \mathbb{B})$ into itself. Then, we conclude that $\mathbb{B}$ is UMD.

When it is assumed that $(i i)$ holds for $B M O_{\circ}((0, \infty), \mathbb{B})$, by proceeding in a similar way, we can prove that $\mathbb{B}$ is UMD.

Thus, the proof of $(i i) \Longrightarrow(i)$ is complete.

4.2. We prove in this section that $(i i i) \Longrightarrow(i)$. In order to see this, we use that the Banach space $\mathbb{B}$ is UMD if, and only if, for every $\gamma \in \mathbb{R} \backslash\{0\}$, the imaginary power $\Delta_{\lambda}^{i \gamma}$ can be extended to $L^{p}((0, \infty), \mathbb{B})$ as a bounded operator from $L^{p}((0, \infty), \mathbb{B})$ into itself, for some (equivalently, for every) $1<p<\infty$ (see [6, Proposition 5.1]).

Let $\gamma \in \mathbb{R} \backslash\{0\}$. We recall that the imaginary power $\Delta_{\lambda}^{i \gamma}$ of $\Delta_{\lambda}$ is defined by

$$
\Delta_{\lambda}^{i \gamma}(f)=h_{\lambda}\left(y^{2 i \gamma} h_{\lambda}(f)\right), \quad f \in L^{2}(0, \infty) .
$$


Since $h_{\lambda}$ is an isometry in $L^{2}(0, \infty), \Delta_{\lambda}^{i \gamma}$ is bounded from $L^{2}(0, \infty)$ into itself. Also, $\Delta_{\lambda}^{i \gamma}$ is a Laplace transform type multiplier for the Bessel operator $\Delta_{\lambda}$, because

$$
y^{2 i \gamma}=y^{2} \int_{0}^{\infty} e^{-y^{2} u} \frac{u^{-i \gamma}}{\Gamma(1-i \gamma)} d u, \quad y \in(0, \infty) .
$$

Laplace transform type Hankel multipliers have been studied in [3] and [15. According to the results established in [3] and [15] the imaginary power $\Delta_{\lambda}^{i \gamma}$ is a Calderón-Zygmund operator defined by the kernel

$$
K_{\lambda}^{\gamma}(x, y)=-\frac{1}{\Gamma(1-i \gamma)} \int_{0}^{\infty} t^{-i \gamma} \partial_{t} W_{t}^{\lambda}(x, y) d t, \quad x, y \in(0, \infty), x \neq y,
$$

where $W_{t}^{\lambda}(x, y)$ is the heat kernel for the Bessel operator $\Delta_{\lambda}$, and it is given by

$$
W_{t}^{\lambda}(x, y)=\frac{(x y)^{1 / 2}}{2 t} I_{\lambda-1 / 2}\left(\frac{x y}{2 t}\right) e^{-\left(x^{2}+y^{2}\right) /(4 t)}, \quad t, x, y \in(0, \infty) .
$$

Here $I_{\nu}$ represents the modified Bessel function of the first class and order $\nu$.

Moreover, the operator $\Delta_{\lambda}^{i \gamma}$ can be extended to $L^{p}(0, \infty)$ as a bounded operator from $L^{p}(0, \infty)$ into itself, for every $1<p<\infty$, from $L^{1}(0, \infty)$ into $L^{1, \infty}(0, \infty)$, and from $H^{1}(0, \infty)$ into $L^{1}(0, \infty)$. This extension, that we continue denoting by $\Delta_{\lambda}^{i \gamma}$, has the following integral representation, for every $f \in L^{p}(0, \infty), 1 \leq p<\infty$,

$$
\Delta_{\lambda}^{i \gamma}(f)(x)=\lim _{\varepsilon \rightarrow 0^{+}}\left(\alpha(\varepsilon) f(x)+\int_{0,|x-y|>\varepsilon}^{\infty} K_{\lambda}^{\gamma}(x, y) f(y) d y\right), \quad \text { a.e. } x \in(0, \infty),
$$

where $\alpha$ is a measurable bounded function.

The operator $\Delta_{\lambda}^{i \gamma}$ is defined on $L^{p}(0, \infty) \otimes \mathbb{B}$ in the natural way.

Let $\gamma \in \mathbb{R} \backslash\{0\}$ and $\beta>0$. We define the operator $T_{\gamma, \beta}$ by

$$
T_{\gamma, \beta}(h)(t)=\frac{1}{t^{\beta}} \int_{0}^{t}(t-s)^{\beta-1} h(t-s) \psi_{\gamma}(s) d s, \quad t>0,
$$

for every $h \in H$, where $\psi_{\gamma}(s)=s^{-2 \gamma i} / \Gamma(1-2 \gamma i), s \in(0, \infty) . T_{\gamma, \beta}$ is a bounded operator from $H$ into itself.

If $f \in S_{\lambda}(0, \infty)$ we proved in [6, (55)] that

$$
G_{\mathbb{C}}^{\lambda, \beta}\left(\Delta_{\lambda}^{i \gamma}(f)\right)(\cdot, x)=-G_{\mathbb{C}}^{\lambda, \beta+1}(f)(\cdot, x) \circ T_{\gamma, \beta}, \quad \text { a.e. } x \in(0, \infty),
$$

as elements of $\gamma(H, \mathbb{C})=H$. Moreover, the two sides of 880 define bounded operators from $L^{p}(0, \infty)$ into $L^{p}((0, \infty), H)$ (see Theorem 1.1 and [42, Theorem 6.2]), for every $1<p<\infty$. Since $S_{\lambda}(0, \infty)$ is dense in $L^{p}(0, \infty)$, we deduce that 88 holds, for every $f \in L^{p}(0, \infty), 1<p<\infty$.

In the following proposition we establish the behaviour of $\Delta_{\lambda}^{i \gamma}$ on $H_{\mathrm{o}}^{1}(0, \infty)$ and $B M O_{\mathrm{o}}(0, \infty)$.

Proposition 4.2. Let $\gamma \in \mathbb{R} \backslash\{0\}$ and $\lambda>0$. The operator $\Delta_{\lambda}^{i \gamma}$ is bounded from $E(0, \infty)$ into itself, where $E$ denotes $H_{\mathrm{o}}^{1}$ or $B M O_{\mathrm{o}}$.

Proof of Proposition 4.2: the case of $E=H_{\mathrm{o}}^{1}$. In order to show that $\Delta_{\lambda}^{i \gamma}$ is bounded from $H_{\mathrm{o}}^{1}(0, \infty)$ into itself we consider the function $\varphi_{\gamma}(x)=x^{2 i \gamma}, x \in(0, \infty)$. It is not hard to see that

$$
\sup _{t>0}\left\|\eta(\cdot) \varphi_{\gamma}(t \cdot)\right\|_{L_{1}^{2}(0, \infty)}<\infty,
$$

where $\eta \in C_{c}^{\infty}(0, \infty)$, the space of smooth functions with compact support on $(0, \infty)$, and $\|\cdot\|_{L_{1}^{2}(0, \infty)}$ denotes the Sobolev norm of order 1 and exponent 2. According to [11, Theorem 4.11] we deduce that $\Delta_{\lambda}^{i \gamma}$ can be extended from $\mathcal{A}=\left\{a \infty\right.$-atom for $\left.H_{\mathrm{o}}^{1}(0, \infty)\right\}$ to $H_{\mathrm{o}}^{1}(0, \infty)$ as a bounded operator from $H_{\mathrm{o}}^{1}(0, \infty)$ into itself. We denote by $\widetilde{\Delta}_{\lambda}^{i \gamma}$ to this extension. 
Suppose that $f=\sum_{j \in \mathbb{N}} \lambda_{j} a_{j}$, where, for every $j \in \mathbb{N}, a_{j}$ is a $\infty$-atom and $\lambda_{j} \in \mathbb{C}$ such that $\sum_{j \in \mathbb{N}}\left|\lambda_{j}\right|<\infty$. We have that

$$
\widetilde{\Delta}_{\lambda}^{i \gamma}(f)=\sum_{j \in \mathbb{N}} \lambda_{j} \Delta_{\lambda}^{i \gamma}\left(a_{j}\right), \text { in } H_{\mathrm{o}}^{1}(0, \infty)
$$

Since $H_{\mathrm{o}}^{1}(0, \infty)$ is continuously contained in $L^{1}(0, \infty)$, the series $\sum_{j=0}^{\infty} \lambda_{j} \Delta_{\lambda}^{i \gamma}\left(a_{j}\right)$ converges also in $L^{1}(0, \infty)$, and then in $L^{1, \infty}(0, \infty)$. Also, the operator $\Delta_{\lambda}^{i \gamma}$ is bounded from $L^{1}(0, \infty)$ into $L^{1, \infty}(0, \infty)$. These facts lead to $\widetilde{\Delta}_{\gamma}^{i \gamma}(f)=\Delta_{\lambda}^{i \gamma}(f)$.

We conclude that $\Delta_{\lambda}^{i \gamma}$ is bounded from $H_{\mathrm{o}}^{1}(0, \infty)$ into itself.

Proof of Theorem 4.2; the case of $E=B M O_{\mathrm{o}}$. In a first step we prove that

$$
\left|K_{\lambda}^{\gamma}(x, y)\right| \leq C \frac{(x y)^{\lambda}}{|x-y|^{2 \lambda+1}}, \quad x, y \in(0, \infty), x \neq y,
$$

being $K_{\lambda}^{\gamma}$ the kernel in 86 .

We will use the following properties of Bessel functions $I_{\nu}, \nu>-1$ (see [30, pp. 108, 110 and 123]):

$$
\begin{gathered}
\frac{d}{d z}\left(z^{-\nu} I_{\nu}(z)\right)=z^{-\nu} I_{\nu+1}, \quad z \in(0, \infty), \\
I_{\nu}(z) \sim \frac{1}{2^{\nu} \Gamma(\nu+1)} z^{\nu}, \text { as } z \rightarrow 0^{+},
\end{gathered}
$$

and

$$
e^{-z} \sqrt{z} I_{\nu}(z)=\mathcal{O}\left(\frac{1}{z}\right)
$$

According to 89 , we have that

$$
\begin{aligned}
\partial_{t} W_{t}^{\lambda}(x, y)= & \partial_{t}\left[\frac{(x y)^{\lambda}}{(2 t)^{\lambda+1 / 2}}\left(\frac{x y}{2 t}\right)^{-\lambda+1 / 2} I_{\lambda-1 / 2}\left(\frac{x y}{2 t}\right) e^{-\left(x^{2}+y^{2}\right) /(4 t)}\right] \\
= & e^{-\left(x^{2}+y^{2}\right) /(4 t)}\left[-(2 \lambda+1)(2 t)^{-\lambda-3 / 2}(x y)^{\lambda}\left(\frac{x y}{2 t}\right)^{-\lambda+1 / 2} I_{\lambda-1 / 2}\left(\frac{x y}{2 t}\right)\right. \\
& -\frac{(x y)^{\lambda}}{(2 t)^{\lambda+1 / 2}} \frac{x y}{2 t^{2}}\left(\frac{x y}{2 t}\right)^{-\lambda+1 / 2} I_{\lambda+1 / 2}\left(\frac{x y}{2 t}\right) \\
& \left.+\frac{(x y)^{\lambda}}{(2 t)^{\lambda+1 / 2}}\left(\frac{x y}{2 t}\right)^{-\lambda+1 / 2} I_{\lambda-1 / 2}\left(\frac{x y}{2 t}\right) \frac{x^{2}+y^{2}}{4 t^{2}}\right], \quad t, x, y \in(0, \infty) .
\end{aligned}
$$

From (91) we deduce that

$$
\begin{aligned}
\left|\partial_{t} W_{t}^{\lambda}(x, y)\right| & \leq C e^{-\left(x^{2}+y^{2}\right) /(4 t)}\left(\frac{(x y)^{\lambda}}{t^{\lambda+3 / 2}}+\frac{(x y)^{\lambda+2}}{t^{\lambda+7 / 2}}+\frac{(x y)^{\lambda}}{t^{\lambda+5 / 2}}\left(x^{2}+y^{2}\right)\right) \\
& \leq C e^{-c\left(x^{2}+y^{2}\right) / t} \frac{(x y)^{\lambda}}{t^{\lambda+3 / 2}}, \quad t, x, y \in(0, \infty) \text { and } x y \leq t
\end{aligned}
$$

and by 92 we get

$$
\begin{aligned}
\left|\partial_{t} W_{t}^{\lambda}(x, y)\right| & \leq C e^{-|x-y|^{2} /(4 t)}\left(\frac{1}{t^{3 / 2}}+\frac{|x-y|^{2}}{t^{5 / 2}}\right) \\
& \leq C \frac{e^{-c|x-y|^{2} / t}}{t^{3 / 2}}, \quad t, x, y \in(0, \infty) \text { and } x y \geq t .
\end{aligned}
$$

Hence,

$$
\left|\partial_{t} W_{t}^{\lambda}(x, y)\right| \leq C e^{-c|x-y|^{2} / t} \frac{(x y)^{\lambda}}{t^{\lambda+3 / 2}}, \quad t, x, y \in(0, \infty),
$$


and then,

$$
\left|K_{\lambda}^{\gamma}(x, y)\right| \leq C(x y)^{\lambda} \int_{0}^{\infty} \frac{e^{-c|x-y|^{2} / t}}{t^{\lambda+3 / 2}} d t \leq C \frac{(x y)^{\lambda}}{|x-y|^{2 \lambda+1}}, \quad x, y \in(0, \infty), x \neq y .
$$

We define the operator $\Delta_{\lambda}^{i \gamma}$ on $B M O_{\mathrm{o}}(0, \infty)$ as follows: if $f \in B M O_{\mathrm{o}}(0, \infty)$ and $\delta>0$,

$$
\Delta_{\lambda}^{i \gamma}(f)(x)=\Delta_{\lambda}^{i \gamma}\left(f \chi_{(0,2 \delta)}\right)(x)+\int_{2 \delta}^{\infty} K_{\lambda}^{\gamma}(x, y) f(y) d y, \text { a.e. } x \in(0, \delta) .
$$

This definition does not depend on $\delta$ (in the suitable sense). By (94) we have that, for every $\delta>0$,

$$
\begin{aligned}
\frac{1}{\delta} \int_{0}^{\delta}\left|\Delta_{\lambda}^{i \gamma}(f)(x)\right| d x & \leq\left(\frac{1}{\delta} \int_{0}^{\delta}\left|\Delta_{\lambda}^{i \gamma}\left(f \chi_{(0,2 \delta)}\right)(x)\right|^{2} d x\right)^{1 / 2}+\frac{C}{\delta} \int_{0}^{\delta} \int_{2 \delta}^{\infty} \frac{(x y)^{\lambda}}{|x-y|^{2 \lambda+1}|f(y)| d y d x} \\
& \leq\left(\frac{1}{\delta} \int_{0}^{2 \delta}|f(y)|^{2} d y\right)^{1 / 2}+\frac{C}{\delta} \int_{0}^{\delta} x^{\lambda} \int_{2 \delta}^{\infty} \frac{|f(y)|}{y^{\lambda+1}} d y d x \\
& \leq C\|f\|_{B M O_{\circ}(0, \infty)}, \quad f \in B M O_{\mathrm{o}}(0, \infty)
\end{aligned}
$$

Hence, $\left|\Delta_{\lambda}^{i \gamma}(f)(x)\right|<\infty$, a.e. $x \in(0, \infty)$, for every $f \in B M O_{\mathrm{o}}(0, \infty)$.

Let $f \in B M O_{\mathrm{o}}(0, \infty)$. We are going to show that $\Delta_{\lambda}^{i \gamma}(f) \in B M O(0, \infty)$. It is wellknown that $B M O(0, \infty)=\left(H^{1}(0, \infty)\right)^{*}$. We denote by $\mathcal{A}(0, \infty)=$ span $\{a$ is $\infty$-atom satisfying $($ Aii $)\}$. Let $g \in \mathcal{A}$. We choose $\delta>0$ such that $\operatorname{supp} g \subset(0, \delta)$. Then,

$$
\Delta_{\lambda}^{i \gamma}(f)(x)=\Delta_{\lambda}^{i \gamma}\left(f \chi_{(0,2 \delta)}\right)(x)+\int_{2 \delta}^{\infty} K_{\lambda}^{\gamma}(x, y) f(y) d y, \text { a.e. } x \in(0, \delta) .
$$

Since $\Delta_{\lambda}^{i \gamma}$ is bounded from $L^{2}(0, \infty)$ into itself, 94 leads to

$$
\begin{aligned}
\int_{0}^{\infty}\left|\Delta_{\lambda}^{i \gamma}(f)(x)\right||g(x)| d x \leq & \int_{0}^{\delta} \mid \Delta_{\lambda}^{i \gamma}\left(f \chi_{(0,2 \delta)}(x)|| g(x) \mid d x\right. \\
& +\int_{0}^{\delta} \int_{2 \delta}^{\infty}\left|K_{\lambda}^{\gamma}(x, y)\right||f(y)| d y|g(x)| d x \\
\leq & \left(\int_{0}^{\delta} \mid \Delta_{\lambda}^{i \gamma}\left(\left.f \chi_{(0,2 \delta)}(x)\right|^{2} d x\right)^{1 / 2}\left(\int_{0}^{\delta}|g(x)|^{2} d x\right)^{1 / 2}\right. \\
& +C \int_{0}^{\delta} \int_{2 \delta}^{\infty} \frac{(x y)^{\lambda}}{|x-y|^{2 \lambda+1}|f(y)| d y|g(x)| d x} \\
\leq & C\left[\left(\frac{1}{\delta} \int_{0}^{2 \delta}|f(x)|^{2} d x\right)^{1 / 2}+\int_{0}^{\delta} x^{\lambda} \int_{2 \delta}^{\infty} \frac{|f(y)|}{y^{\lambda+1}} d y d x\right] \\
\leq & \left.C\|f\|_{B M O_{\circ}(0, \infty)}\right]
\end{aligned}
$$

because $g \in L^{\infty}(0, \infty)$.

We define the functional $\mathcal{L}$ on $\mathcal{A}$ by

$$
\mathcal{L}(g)=\int_{0}^{\infty} \Delta_{\lambda}^{i \gamma}(f)(x) g(x) d x, \quad g \in \mathcal{A} .
$$

Let $g \in \mathcal{A}$ such that supp $g \subset(0, \delta)$ with $\delta>0$. We can write

$$
\mathcal{L}(g)=\int_{0}^{\infty} \Delta_{\lambda}^{i \gamma}\left(f \chi_{(0,2 \delta)}\right)(x) g(x) d x+\int_{0}^{\infty} g(x) \int_{2 \delta}^{\infty} K_{\lambda}^{\gamma}(x, y) f(y) d y d x .
$$

Since, as it was seen,

$$
\int_{0}^{\infty}|g(x)| \int_{2 \delta}^{\infty}\left|K_{\lambda}^{\gamma}(x, y)\right||f(y)| d y d x<\infty
$$


according to 87 we get

$$
\int_{0}^{\infty} g(x) \int_{2 \delta}^{\infty} K_{\lambda}^{\gamma}(x, y) f(y) d y d x=\int_{2 \delta}^{\infty} f(y) \int_{0}^{\delta} K_{\lambda}^{\gamma}(x, y) g(x) d x d y=\int_{2 \delta}^{\infty} f(y) \Delta_{\lambda}^{i \gamma}(g)(y) d y .
$$

By using Plancherel's equality for Hankel transforms it follows that

$$
\int_{0}^{\infty} g(x) \Delta_{\lambda}^{i \gamma}\left(f \chi_{(0,2 \delta)}\right)(x) d x=\int_{0}^{2 \delta} f(y) \Delta_{\lambda}^{i \gamma}(g)(y) d y
$$

Hence,

We also have that

$$
\mathcal{L}(g)=\int_{0}^{\infty} f(y) \Delta_{\lambda}^{i \gamma}(g)(y) d y
$$

$$
\begin{aligned}
\int_{0}^{\infty}\left|f(y) \Delta_{\lambda}^{i \gamma}(g)(y)\right| d y & \leq \int_{0}^{2 \delta}\left|f(y)\left\|\Delta_{\lambda}^{i \gamma}(g)(y)\left|d y+\int_{2 \delta}^{\infty}\right| f(y)\left|\int_{0}^{\delta}\right| K_{\lambda}^{\gamma}(x, y)\right\| g(x)\right| d x d y \\
& \leq\left(\int_{0}^{2 \delta}|f(y)|^{2} d y\right)^{1 / 2}\left\|\Delta_{\lambda}^{i \gamma}(g)\right\|_{L^{2}(0, \infty)}+C \int_{2 \delta}^{\infty} \frac{\delta^{\lambda+1}}{y^{\lambda+1}}|f(y)| d y \\
& \leq C\left(\sqrt{\delta}\|g\|_{L^{2}(0, \infty)}+1\right)\|f\|_{B M O_{\circ}(0, \infty)} \leq C\|f\|_{B M O_{\circ}(0, \infty)} .
\end{aligned}
$$

According to [4, Proposition 2.5] adapted to this Bessel setting (see also [17]), since $\Delta_{\lambda}^{i \gamma}$ is bounded from $H_{\mathrm{o}}^{1}(0, \infty)$ into itself, we deduce

$$
|\mathcal{L}(g)| \leq C\|f\|_{B M O_{\circ}(0, \infty)}\left\|\Delta_{\lambda}^{i \gamma}(g)\right\|_{H_{\mathrm{o}}^{1}(0, \infty)} \leq C\|f\|_{B M O_{\mathrm{o}}(0, \infty)}\|g\|_{H_{\mathrm{o}}^{1}(0, \infty)} .
$$

Here $C>0$ does not depend on $g$.

We conclude that $\Delta_{\lambda}^{i \gamma}(f) \in B M O_{\mathrm{o}}(0, \infty)$ and $\left\|\Delta_{\lambda}^{i \gamma}(f)\right\|_{B M O_{\circ}(0, \infty)} \leq C\|f\|_{B M O_{\circ}(0, \infty)}$.

Suppose now that $($ iii $)$ holds in the Hardy setting. Let $f \in H_{\mathrm{o}}^{1}(0, \infty) \otimes \mathbb{B}$. According to Proposition 4.2. $\Delta_{\lambda}^{i \gamma}(f) \in H_{\mathrm{o}}^{1}(0, \infty) \otimes \mathbb{B}$. Then, by using 88 and by taking into account [42, Theorem 6.2] we get

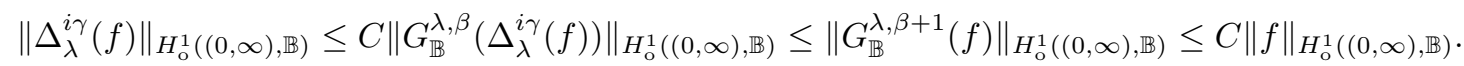

According to [31, Theorem 4.1] we deduce that $\Delta_{\lambda}^{i \gamma}$ can be extended to $L^{p}((0, \infty), \mathbb{B})$ into itself, for every $1<p<\infty$.

Hence, by [6, Proposition 5.1] we conclude that $\mathbb{B}$ is a UMD space.

By proceeding in a similar way we can prove that $\mathbb{B}$ is a UMD space provided that ( $i$ ii $)$ holds in the BMO situation.

Thus, the proof of this theorem is finished.

\section{REFERENCES}

[1] I. Abu-Falahah, P. R. Stinga, and J. L. Torrea, Square functions associated to Schrödinger operators, Studia Math., 203 (2011), pp. 171-194.

[2] J. J. Betancor, D. Buraczewski, J. C. Fariña, T. Martínez, and J. L. Torrea, Riesz transforms related to Bessel operators, Proc. Roy. Soc. Edinburgh Sect. A, 137 (2007), pp. 701-725.

[3] J. J. Betancor, A. J. Castro, and J. Curbelo, Spectral multipliers for multidimensional Bessel operators, J. Fourier Anal. Appl., 17 (2011), pp. 932-975.

[4] J. J. Betancor, A. J. Castro, J. Curbelo, J. C. Fariña, and L. Rodríguez-Mesa, $\gamma$-radonifying operators and UMD-valued Littlewood-Paley-Stein functions in the Hermite setting on BMO and Hardy spaces, J. Funct. Anal., 263 (2012), pp. 3804-3856.

[5] - Square functions in the Hermite setting for functions with values in UMD spaces, to appear in Annali di Matematica Pura ed Applicata (DOI: 10.1007/s10231-013-0335-9). 
[6] J. J. Betancor, A. J. Castro, and L. Rodríguez-Mesa, Square functions and spectral multipliers for Bessel operators in UMD spaces, preprint 2013 (arXiv:1303.3159v1)

[7] J. J. Betancor, A. J. Castro, And P. Stinga, The fractional Bessel equation in Hölder spaces, preprint 2013.

[8] J. J. Betancor, A. Chicco Ruiz, J. C. Fariña, and L. Rodríguez-Mesa, Maximal operators, Riesz transforms and Littlewood-Paley functions associated with Bessel operators on BMO, J. Math. Anal. Appl., 363 (2010), pp. 310-326.

[9] - Odd $\mathrm{BMO}(\mathbb{R})$ functions and Carleson measures in the Bessel setting, Integral Equations Operator Theory, 66 (2010), pp. 463-494.

[10] J. J. Betancor, R. Crescimbeni, J. C. Fariña, and L. Rodríguez-Mesa, Multipliers and imaginary powers of the Schrödinger operators characterizing UMD Banach spaces, Ann. Acad. Sci. Fenn., 38 (2013), 209-227.

[11] J. J. Betancor, J. Dziubański, and J. L. Torrea, On Hardy spaces associated with Bessel operators, J. Anal. Math., 107 (2009), pp. 195-219.

[12] J. J. Betancor, J. C. Fariña, E. Harboure, and L. Rodríguez-Mesa, Variation operators for semigroups and Riesz transforms on BMO in the Schrödinger setting, to appear in Potential Analysis (DOI: $10.1007 /$ s11118-012-9294-9).

[13] J. J. Betancor, J. C. Fariña, T. Martínez, and J. L. Torrea, Riesz transform and g-function associated with Bessel operators and their appropriate Banach spaces, Israel J. Math., 157 (2007), pp. 259282.

[14] J. J. Betancor, J. C. Fariña, and A. Sanabria, On Littlewood-Paley functions associated with Bessel operators, Glasg. Math. J., 51 (2009), pp. 55-70.

[15] J. J. Betancor, T. Martínez, and L. Rodríguez-Mesa, Laplace transform type multipliers for Hankel transforms, Canad. Math. Bull., 51 (2008), pp. 487-496.

[16] J. J. Betancor and K. Stempak, On Hankel conjugate functions, Studia Sci. Math. Hungar., 41 (2004), pp. 59-91.

[17] A. Bonami, T. Iwaniec, P. Jones, And M. Zinsmeister, On the product of functions in BMO and $H^{1}$, Ann. Inst. Fourier, Grenoble, 57 (2007), pp. 1405-1439.

[18] J. Bourgain, Some remarks on Banach spaces in which martingale difference sequences are unconditional, Ark. Mat., 21 (1983), pp. 163-168.

[19] A. Buknalov, Sobolev spaces of vector-valued functions, J. Math. Sciences, 71 (1994), pp. 2173-2179.

[20] D. L. Burkholder, A geometrical characterization of Banach spaces in which martingale difference sequences are unconditional, Ann. Probab., 9 (1981), pp. 997-1011.

[21] T. Coulhon and D. Lamberton, Régularité $L^{p}$ pour les équations d'évolution, in Séminaire d'Analyse Fonctionelle 1984/1985, vol. 26 of Publ. Math. Univ. Paris VII, Paris, 1986, pp. 155-165.

[22] A. Erdélyi et al., Tables of integral transforms, Volume II, McGraw-Hill, Inc., New York, 1954.

[23] S. FriduI, Hardy spaces generated by an integrability condition, J. Approx. Theory, 113 (2001), pp. 91-109.

[24] P. Graczyk, J. J. Loeb, I. A. López P., A. Nowak, and W. O. Urbina R., Higher order Riesz transforms, fractional derivatives, and Sobolev spaces for Laguerre expansions, J. Math. Pures Appl. (9), 84 (2005), pp. 375-405.

[25] S. Guerre-Delabrière, Some remarks on complex powers of $(-\Delta)$ and UMD spaces, Illinois J. Math., 35 (1991), pp. 401-407.

[26] T. Hytönen, Littlewood-Paley-Stein theory for semigroups in UMD spaces, Rev. Mat. Iberoam., 23 (2007), pp. 973-1009.

[27] C. KAISER, Wavelet transforms for functions with values in Lebesgue spaces, in Wavelets XI, vol. 5914 of Proc. of SPIE, Bellingham, WA, 2005.

[28] C. Kaiser And L. Weis, Wavelet transform for functions with values in UMD spaces, Studia Math., 186 (2008), pp. 101-126.

[29] S. KWAPIEŃ, Isomorphic characterizations of inner product spaces by orthogonal series with vector valued coefficients, Studia Math., 44 (1972), pp. 583-595.

[30] N. N. Lebedev, Special functions and their applications, Dover Publications Inc., New York, 1972.

[31] T. Martínez, J. L. Torrea, and Q. Xu, Vector-valued Littlewood-Paley-Stein theory for semigroups, Adv. Math., 203 (2006), pp. 430-475. 
[32] S. Meda, A general multiplier theorem, Proc. Amer. Math. Soc., 110 (1990), pp. 639-647.

[33] B. Muckenhoupt and E. M. Stein, Classical expansions and their relation to conjugate harmonic functions, Trans. Amer. Math. Soc., 118 (1965), pp. 17-92.

[34] J. L. Rubio de Francia, F. J. Ruiz, and J. L. Torrea, Calderón-Zygmund theory for operator-valued kernels, Adv. in Math., 62 (1986), pp. 7-48.

[35] H. J. Schmeisser And W. Sickel, Vector-valued Sobolev spaces and Gagliardo-Nirenberg inequalities, in Nonlinear elliptic and parabolic problems, vol. 64 of Progr. Nonlinear Differential Equations Appl., Birkhäuser, Basel, 2005, pp. 463-472.

[36] C. Segovia And R. L. Wheeden, On certain fractional area integrals, J. Math. Mech., 19 (1969/1970), pp. 247-262.

[37] E. M. Stein, Topics in harmonic analysis related to the Littlewood-Paley theory, Annals of Mathematics Studies, No. 63, Princeton University Press, Princeton, N.J., 1970.

[38] - Harmonic analysis: real-variable methods, orthogonality, and oscillatory integrals, vol. 43 of Princeton Mathematical Series, Princeton University Press, Princeton, NJ, 1993.

[39] K. Stempak, The Littlewood-Paley theory for the Fourier-Bessel transform, PhD thesis, Mathematical Institute University of Wroclaw, Poland, 1985.

[40] A. Torchinsky, Real-variable methods in harmonic analysis, vol. 123 of Pure and Applied Mathematics, Academic Press Inc., Orlando, FL, 1986.

[41] J. L. Torrea And C. Zhang, Fractional vector-valued Littlewood-Paley-Stein theory for semigroups. Preprint 2011 (arXiv:1105.6022 v3)

[42] J. van NeERVEn, $\gamma$-radonifying operators - a survey, in The AMSI-ANU Workshop on Spectral Theory and Harmonic Analysis, vol. 44 of Proc. Centre Math. Appl. Austral. Nat. Univ., Canberra, 2010, pp. 1-61.

[43] J. van Neerven, M.C. Veraar, and L. Weis, Stochastic evolution equations in UMD Banach spaces, J. Funct. Anal., 255 (2008), pp. 940-993.

[44] Q. Xu, Littlewood-Paley theory for functions with values in uniformly convex spaces, J. Reine Angew. Math., 504 (1998), pp. 195-226.

[45] A. H. Zemanian, A distributional Hankel transformation, SIAM J. Appl. Math., 14 (1966), pp. 561-576.

Jorge J. Betancor, Alejandro J. Castro and Lourdes Rodríguez-Mesa

Departamento de Análisis Matemático, Universidad de la laguna,

Campus de Anchieta, Avda. Astrofísico Francisco Sánchez, s/n,

38271, La Laguna (Sta. Cruz de Tenerife), Spain

E-mail address: jbetanco@ull.es, ajcastro@ull.es, lrguez@ull.es 